\title{
Isospecific Group Transfer Polymerization of Diethyl Vinylphosphonate and Multidimensional NMR Analysis of the Polymer Microstructure
}

Michael Weger, ${ }^{1, \ddagger}$ Philipp Pahl, ${ }^{1,}$ Fabian Schmidt, ${ }^{1, \ddagger}$ Benedikt S. Soller, ${ }^{1}$ Philipp J. Altmann, ${ }^{2}$ Alexander Pöthig, ${ }^{2}$ Gerd Gemmecker, ${ }^{3}$ Wolfgang Eisenreich, ${ }^{4, *}$ and Bernhard Rieger ${ }^{1, *}$

${ }^{1}$ Catalysis Research Center \& WACKER-Chair of Macromolecular Chemistry, Technical University of Munich, Lichtenbergstraße 4, D-85747 Garching near Munich

${ }^{2}$ Catalysis Research Center \& Chair of Inorganic and Metal-Organic Chemistry, Technical University of Munich, Lichtenbergstraße 4, D-85747 Garching near Munich

${ }^{3}$ Bavarian NMR Center, Technical University of Munich, Lichtenbergstraße 4, D-85747 Garching near Munich

${ }^{4}$ Chair of Biochemistry, Technical University of Munich, Lichtenbergstraße 4, D-85747 Garching near Munich

* Corresponding Author: rieger@tum.de, wolfgang.eisenreich@mytum.de

† These authors contributed equally.

1. Experimental Section

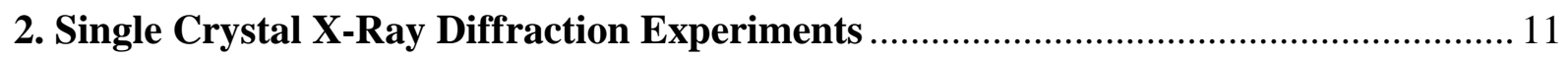

3. Polymerization Studies and Mechanism Elucidation ................................................ 20

4. NMR, GPC and Thermal Analysis of the PDEVP Samples ........................................ 25

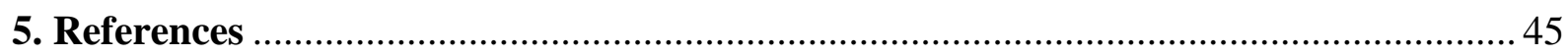




\section{Experimental Section}

\section{Materials and Methods:}

All reactions were carried out under argon atmosphere using standard Schlenk or glovebox techniques. All glassware was heat dried under vacuum prior to use. Unless otherwise stated, all chemicals were purchased from Sigma-Aldrich, VWR-International or ABCR and used as received. Toluene and tetrahydrofuran were dried using an MBraun SPS-800 solvent purification system. DEVP was dried over $\mathrm{CaH}_{2}$ and destilled prior to use.

NMR spectra were recorded at $300 \mathrm{~K}$ on a Bruker AV-NEO 400 spectrometer equipped with a triple resonance TBI-probe, the ${ }^{13} \mathrm{C}\left\{{ }^{1} \mathrm{H}\right\}$ spectra on a Bruker AV-III-500 spectrometer equipped with a QNP-Cryoprobe and the ${ }^{1} \mathrm{H}\left\{{ }^{31} \mathrm{P}\right\}$ spectra on a Bruker AV-III-600 spectrometer equipped with a QCI-Cryoprobe. The details (acquisition parameters, pulse program) for the triple resonance experiments can be found in section $4 .{ }^{1} \mathrm{H}$ NMR spectroscopic chemical shifts $\delta$ are reported in ppm relative to tetramethylsilane. $\delta\left({ }^{1} \mathrm{H}\right)$ is calibrated to the residual proton signal of the solvent. Deuterated solvents were obtained from Eurisotop or Sigma Aldrich.

For the ESI-MS analysis a Varian 500 LC MS ion trap spectrometer was used with acetonitrile as carrier $(20 \mu \mathrm{l} / \mathrm{min})$.

GPC was carried out on a Varian LC-920 equipped with two PL Polargel columns using tetrahydrofuran/water (1:1, $9 \mathrm{~g} / \mathrm{L}$ tetrabutylammonium bromide) as eluent. Absolute molecular weights have been determined multiangle laser light scattering (MALLS) analysis (LC-920) using a Wyatt Dawn Heleos II in combination with a Wyatt Optilab rEX as concentration detector, coupled with GPC.

Elemental analysis was performed at the microanalytic laboratory of the Department of Inorganic Chemistry at the Technical University of Munich.

DSC measurements were carried out at a DSC G2000 of TA instruments with a heating rate of $1{ }^{\circ} \mathrm{C} / \mathrm{min}$ in the first and $20^{\circ} \mathrm{C} / \mathrm{min}$ in the third cycle and a cooling rate of $5{ }^{\circ} \mathrm{C} / \mathrm{min}$.

Thermal gravimetric analysis (TGA) of the materials was carried out with a NETZSCH STA449 F5 Jupiter machine using aluminum oxide pans (70 $\mu \mathrm{L}$ with lid) with sample amounts of 1 to $5 \mathrm{mg}$. Temperature calibration of the oven cell was performed on the basis of the following metals: In, Sn, Bi, $\mathrm{Zn}, \mathrm{Al}$ and $\mathrm{Au}$. The baseline was corrected screening an empty sample pan with the respective measurement program prior to the experiment. The following thermal program was applied using argon gas $(20 \mathrm{~mL} / \mathrm{min})$ for purging: At $27^{\circ} \mathrm{C}$ isothermal 
equilibration (30 min), ramp from $27^{\circ} \mathrm{C}$ to $800{ }^{\circ} \mathrm{C}$ with $10 \mathrm{~K} / \mathrm{min}$, isothermal equilibrium for 5 minutes.

\section{General procedure for the polymerization of DEVP:}

Polymerizations were performed in $30 \mathrm{~mL}$ oven-dried glass reactors interfaced to a dualmanifold Schlenk line at various temperatures under argon atmosphere. A predetermined amount of a catalyst was first dissolved in toluene. Then the polymerization was started by addition of the monomer $(1 \mathrm{~mL})$ via a gastight syringe under vigorous stirring. After the measured time interval, a $0.2 \mathrm{~mL}$ aliquot was taken from the reaction mixture via a syringe and quickly quenched into a $4 \mathrm{~mL}$ vial containing $0.4 \mathrm{~mL}$ of undried "wet" MeOD- $\mathrm{d}_{4}$. The quenched aliquots were later analyzed by ${ }^{1} \mathrm{H}-\mathrm{NMR}$ and ${ }^{31} \mathrm{P}-\mathrm{NMR}$ to obtain the percent polymer yield data. The polymerization was immediately quenched after the removal of the aliquot by addition of $0.5 \mathrm{~mL}$ methanol. The solvents were evaporated from the quenched mixtures under reduced pressure, the polymers were purified by precipitation in pentane and dried in a vacuum oven at $50{ }^{\circ} \mathrm{C}$ overnight to a constant weight.

\section{Activity measurements:}

The stated amount of catalyst is dissolved in toluene and the reaction mixture is thermostated to the desired temperature. Then, the stated amount of monomer is added. During the course of the measurement, the temperature is monitored with a digital thermometer and aliquots $(0.5 \mathrm{ml})$ are taken and quenched by addition to MeOD- $\mathrm{d}_{4}(0.2 \mathrm{ml})$. After the stated reaction time, the reaction is quenched by addition of $\mathrm{MeOH}(0.5 \mathrm{ml})$. The procedure was performed at least twice for every polymerization to obtain accurate activity values The TOFs are calculated corresponding to the following equation: ${ }^{1}$

$$
T O F=\frac{n(\text { Mon })}{n(\text { Cat }) \times t}=\frac{n(\text { Mon })_{0} \times X}{n(\text { Cat }) \times t}
$$

\section{Characterization of Synthesized Organometallic Compounds:}

The ligands $\left(\mathrm{C}_{5} \mathrm{Me}_{4} \mathrm{H}\right) \mathrm{CH}_{2} \mathrm{CH}_{2} \mathrm{NH} t \mathrm{Bu},{ }^{2}\left(\mathrm{C}_{9} \mathrm{H}_{7}\right) \mathrm{SiMe}_{2} \mathrm{NH} t \mathrm{Bu}^{3},{ }^{3}\left(\mathrm{C}_{5} \mathrm{Me}_{4} \mathrm{H}\right) \mathrm{SiMe}_{2} \mathrm{NHPh}^{4}$ and $\left(\mathrm{C}_{5} \mathrm{Me}_{4} \mathrm{H}\right) \mathrm{SiMe}_{2} \mathrm{NH} t \mathrm{Bu}^{5}$ were synthesized according to literature procedures.

LiCH2 TMS $1.72 \mathrm{~g}$ Lithium granulate (249 mmol, 3.3 eq.) and $9.25 \mathrm{~g}$ chloromethyltrimethylsilane $(75.0 \mathrm{mmol}, 1$ eq.) are suspended in $100 \mathrm{~mL}$ hexane and the mixture is heated at $35{ }^{\circ} \mathrm{C}$ for $24 \mathrm{~h}$. Lithium is exempt of lithiumchloride several times using an ultrasonic bath. The supernatant solution is isolated using a filter cannula. The residue is 
extracted three times with hexane $(15 \mathrm{ml})$ and the solvent is removed in vacuo, yielding a white pyrophoric solid (6.25 g, $66.0 \mathrm{mmol}, 88 \%) .{ }^{1} \mathrm{H}$ NMR (400 MHz, $\left.\mathrm{C}_{6} \mathrm{D}_{6}\right) \delta=0.16(\mathrm{~s}, 9 \mathrm{H}),-2.08$ $(\mathrm{s}, 2 \mathrm{H}) .{ }^{13} \mathrm{C}$ NMR $\left(126 \mathrm{MHz}, \mathrm{C}_{6} \mathrm{D}_{6}\right) \delta=3.6,-4.7$. elemental analysis calcd (\%) for $\mathrm{C}_{4} \mathrm{H}_{11} \mathrm{LiSi}$ : C, 51.03; H, 11.78. Found: C, 51.14; H, 12.00.

$\mathbf{Y C l}_{3}$ (thf) 3.5 $_{\text {. }}$ A glass thimble is charged inside a glovebox to $2 / 3$ with yttrium(III) chloride and is placed in a Soxhlet extractor. All glass joints of the reaction setup are diligently sealed using Teflon grease. The Soxhlet extractor is attached to a Schlenk flask with $150 \mathrm{~mL}$ THF, a reflux condenser, and a pressure valve using a Schlenk line outside the glovebox. The oil bath is heated at $110{ }^{\circ} \mathrm{C}$ and $\mathrm{THF}$ is heated to reflux for $36 \mathrm{~h}$ under vigorous stirring. After cooling the Schlenk flask to room temperature and detaching it from the reaction setup, THF is removed in vacuo. The product is obtained as a white powder and the amount of coordinating THF is determined by elemental analysis. The yield is nearly quantitative if pure yttrium (III)chloride is used as a starting material. Elemental analysis calcd. for $\mathrm{YCl}_{3}(\text { thf })_{3.5}: \mathrm{C} 37.56, \mathrm{H}$ 6.30. Found: C 37.45, H 6.33 .

$\mathbf{Y C H}_{2} \mathbf{T M S}_{3}$ (thf) $)_{2} 1.79 \mathrm{~g} \mathrm{YCl}_{3}(\text { thf })_{3.5}(4.00 \mathrm{mmol}, 1$ eq. $)$ is suspended in $25 \mathrm{~mL}$ pentane. A solution of $1.13 \mathrm{~g}$ trimethylsilylmethyl lithium ( $12 \mathrm{mmol}, 3 \mathrm{eq}$.$) in 35 \mathrm{~mL}$ pentane is added dropwise at $0{ }^{\circ} \mathrm{C}$ and the reaction solution is stirred at $0{ }^{\circ} \mathrm{C}$ for $2 \mathrm{~h}$. The supernatant solution is isolated using a filter cannula and the residue is extracted with pentane $(2 \times 10 \mathrm{~mL})$. The solvent is removed in vacuo and the product is obtained as a white solid $(1.78 \mathrm{~g}, 3.60 \mathrm{mmol}, 90 \%) .{ }^{1} \mathrm{H}$ NMR (400 MHz, $\left.\mathrm{C}_{6} \mathrm{D}_{6}\right) \delta=4.01-3.87$ (m, 8 H, THF-H), 1.36-1.21 (m, 8 H, THF-H), 0.31 (s, $\left.27 \mathrm{H}, \mathrm{CH}_{2} \mathrm{SiMe}_{3}\right),-0.67$ (d, $\left.6 \mathrm{H}, \mathrm{CH}_{2} \mathrm{SiMe}_{3}\right) .{ }^{13} \mathrm{C} \mathrm{NMR}\left(126 \mathrm{MHz}, \mathrm{C}_{6} \mathrm{D}_{6}\right) \delta=69.8,33.8,25.2$, 4.5 .

$\left(\mathrm{C}_{5} \mathrm{Me}_{4}\right) \mathrm{Me}_{2} \mathrm{SiN} t \mathrm{BuYCH} \mathrm{CH}_{2} \mathrm{TMS}(\mathrm{thf})$ was synthesized according to an literature procedure. ${ }^{6}$ Recrystallization from pentane yields a colorless powder (2.29 g, $4.60 \mathrm{mmol}, 52 \%) .{ }^{1} \mathrm{H} \mathrm{NMR}$ $\left(400 \mathrm{MHz}, \mathrm{C}_{6} \mathrm{D}_{6}\right) \delta=3.38-3.15(\mathrm{~m}, 4 \mathrm{H}, \mathrm{THF}-\mathrm{H}), 2.21\left(\mathrm{~s}, 12 \mathrm{H}, \mathrm{CH}_{3}\right), 1.39$ (s, 9H, NC(CH3) 3 ), 1.03-0.96 (m, 4H, THF-H), 0.77 (s, 6H, $\mathrm{SiMe}_{2}$ ), 0.31 (s, 9H, $\left.\mathrm{CH}_{2} \mathrm{SiMe}_{3}\right),-0.90$ (d, ${ }^{1} \mathrm{~J}=3.2 \mathrm{~Hz}$, $\left.2 \mathrm{H}, \mathrm{YCH}_{2}\right) .{ }^{13} \mathrm{C} \mathrm{NMR}\left(126 \mathrm{MHz}, \mathrm{C}_{6} \mathrm{D}_{6}\right) \delta=126.4,122.3,106.6,70.7,54.0,36.0,26.2,24.7$, 14.0, 11.5, 8.4, 4.7. ${ }^{29} \mathrm{Si} \mathrm{NMR}\left(99 \mathrm{MHz}, \mathrm{C}_{6} \mathrm{D}_{6}\right) \delta=-2.73,-25.06$. Anal. Calcd for $\mathrm{C}_{23} \mathrm{H}_{46} \mathrm{NOSi}_{2} \mathrm{Y}: \mathrm{C}, 55.51 ; \mathrm{H}, 9.32 ; \mathrm{N}, 2.81$. Found: C, 55.77; H, 9.50; N, 2.85.

$\left(\mathrm{C}_{5} \mathrm{Me}_{4}\right) \mathrm{CH}_{2} \mathrm{CH}_{2} \mathrm{~N} t$ BuYCH $\mathbf{T M S}($ thf $) \quad 988 \mathrm{mg} \quad \mathrm{Y}\left(\mathrm{CH}_{2} \mathrm{TMS}\right)_{3}(\text { thf })_{2} \quad(1.89 \mathrm{mmol}, 1$ eq. $)$ is dissolved in $18 \mathrm{~mL}$ pentane and the mixture is cooled to $0{ }^{\circ} \mathrm{C}$. A solution of $418 \mathrm{mg}$ $\left(\mathrm{C}_{5} \mathrm{Me}_{4} \mathrm{H}\right) \mathrm{CH}_{2} \mathrm{CH}_{2} \mathrm{NH} t \mathrm{Bu}$ (1.89 mmol, 1 eq.) in $7 \mathrm{~mL}$ pentane is added dropwise and the reaction solution is stirred for two hours at $0{ }^{\circ} \mathrm{C}$. Volatile compounds are removed in vacuo, 
resulting in a yellow solid. The crude product is washed with cold pentane and dried in vacuo. The product is purified by recrystallization from pentane and is obtained as a slightly yellow crystalline solid (787 mg, $1.68 \mathrm{mmol}, 89 \%) .{ }^{1} \mathrm{H}$ NMR (400 MHz, $\left.\mathrm{C}_{6} \mathrm{D}_{6}\right) \delta=3.89-3.81$ (m, 2H, $\mathrm{CH}_{2} \mathrm{CH}_{2} \mathrm{~N}$ ), 3.60-3.20 (m, 4H, THF-H), $3.08-3.01$ (t, $\left.2 \mathrm{H}, \mathrm{CH}_{2} \mathrm{CH}_{2} \mathrm{~N}\right), 2.25-1.96(\mathrm{~m}, 12 \mathrm{H}$, $\mathrm{CH}_{3}$ ), 1.34 (s, 9H, NC(CH3) $\left.)_{3}\right), 1.08-0.97$ (m, 4H, THF-H), 0.36 (s, 9H, $\mathrm{CH}_{2} \mathrm{SiMe}_{3}$ ), -0.97 (d, $\left.2 \mathrm{H}, \mathrm{YCH}_{2}\right) .{ }^{13} \mathrm{C} \mathrm{NMR}\left(126 \mathrm{MHz}, \mathrm{C}_{6} \mathrm{D}_{6}\right) \delta=126.4,126.2,126.0,68.5,53.7,52.4,28.2,25.8$, 23.2, 22.7, 9.1, 3.0, -2.0. ${ }^{29} \mathrm{Si}$ NMR $\left(99 \mathrm{MHz}, \mathrm{C}_{6} \mathrm{D}_{6}\right) \delta=-2.86$. elemental analysis calcd $(\%)$ for $\mathrm{C}_{23} \mathrm{H}_{44} \mathrm{NOSiY}$ : C 59.08, H 9.48, N 3.00. Found: C 58.70, H 9.43, N 3.17.

$\left(\mathrm{C}_{5} \mathrm{Me}_{4}\right) \mathrm{Me}_{2} \mathrm{SiNPhYCH}$ TMS(thf $)_{2} 959 \mathrm{mg} \quad \mathrm{Y}_{2}\left(\mathrm{CH}_{2} \mathrm{TMS}\right)_{3}(\mathrm{thf})_{2} \quad(1.96 \mathrm{mmol}, \quad 1.1 \mathrm{eq}$.$) is$ dissolved in $10 \mathrm{~mL}$ hexane and the mixture is cooled to $0{ }^{\circ} \mathrm{C}$. A solution of $480 \mathrm{mg}$ $\left(\mathrm{C}_{5} \mathrm{Me}_{4} \mathrm{H}\right) \mathrm{SiMe}_{2} \mathrm{NHPh}(1.77 \mathrm{mmol}, 1$ eq. $)$ in $5 \mathrm{~mL}$ hexane is added dropwise and the reaction solution is stirred for two hours at r.t. After filtration the residue is washed with pentane $(2 \times 5$ $\mathrm{mL}$ ) and dried in vacuo. The product is purified by diffusion crystallization in toluene/pentane at $-35^{\circ} \mathrm{C}$ and obtained as a colorless crystalline solid (870 mg, $\left.1.49 \mathrm{mmol}, 84 \%\right) .{ }^{1} \mathrm{H}$ NMR (400 $\left.\mathrm{MHz}, \mathrm{C}_{6} \mathrm{D}_{6}\right) \delta=7.27-7.20$ (m, 2H, Ph-H), 6.73-6.64 (m, 3H, Ph-H), 3.69-3.53 (m, 8H, THFH), $2.11\left(\mathrm{~s}, 6 \mathrm{H}, \mathrm{CH}_{3}\right), 2.06\left(\mathrm{~s}, 6 \mathrm{H}, \mathrm{CH}_{3}\right), 1.42-1.31$ (m, 8H, THF-H), 0.85 (s, 6H, $\left.\mathrm{SiMe}_{2}\right), 0.25$ (s, 9H, $\left.\mathrm{CH}_{2} \mathrm{SiMe}\right),-1.07\left(\mathrm{~d},{ }^{1} \mathrm{~J}=2.6 \mathrm{~Hz}, 2 \mathrm{H}, \mathrm{YCH}_{2}\right) .{ }^{13} \mathrm{C} \mathrm{NMR}\left(126 \mathrm{MHz}, \mathrm{C}_{6} \mathrm{D}_{6}\right) \delta=156.6$, 129.4, 126.8, 122.6, 119.6, 114.5, 105.6, 68.2, 25.3, 21.4, 13.7, 11.4, 4.7, 4.3. ${ }^{29} \mathrm{Si}$ NMR (99 $\left.\mathrm{MHz}, \mathrm{C}_{6} \mathrm{D}_{6}\right) \delta=-2.44,-23.69$. elemental analysis calcd (\%) for $\mathrm{C}_{29} \mathrm{H}_{50} \mathrm{NO}_{2} \mathrm{Si}_{2} \mathrm{Y}: \mathrm{C}, 59.06 ; \mathrm{H}$, 8.55; N, 2.37. Found: C, 58.75; H, 8.25; N, 2.29 .

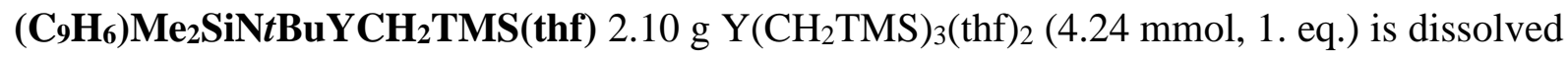
in $50 \mathrm{~mL}$ pentane and the mixture is cooled to $0{ }^{\circ} \mathrm{C}$. A solution of $1.04 \mathrm{mg}\left(\mathrm{C}_{9} \mathrm{H}_{7}\right) \mathrm{SiMe}_{2} \mathrm{NH} t \mathrm{Bu}$ (4.24 mmol, 1 eq.) in $8 \mathrm{~mL}$ pentane is added dropwise and the reaction solution is stirred over night at r.t. After filtration the filtrate is reduced to $10 \mathrm{~mL}$ and the product precipitates at $-78{ }^{\circ} \mathrm{C}$ as a colorless crystalline solid (470 mg, $960 \mu \mathrm{mol}, 23 \%$ ). After drying the solid in vacuo, the known instability can be observed. ${ }^{7}{ }^{1} \mathrm{H}$ NMR (400 MHz, $\left.\mathrm{C}_{6} \mathrm{D}_{6}\right) \delta=7.89-7.81$ (m, $1 \mathrm{H}, \mathrm{H}_{\text {Ind }}$ ), $7.54-7.46$ (m, 1H, $\left.\mathrm{H}_{\text {Ind }}\right), 7.15$ - 7.12 (m, 1H, $\mathrm{H}_{\text {Ind }}$ ), $7.06-7.01$ (m, 1H, $\mathrm{H}_{\text {Ind }}$ ), 6.79-6.70 (m, 2H, $\left.\mathrm{H}_{\text {Ind }}\right), 2.95-2.82$ (m, 4H, THF-H), 1.30 (s, 9H, NC(CH3) $\left.)_{3}\right), 0.91$ (s, 3H, SiMe $), 0.88-0.82$ (m, 4H, THF-H), 0.80 (s, 3H, $\mathrm{SiMe}_{2}$ ), 0.38 (s, 9H, $\mathrm{CH}_{2} \mathrm{SiMe}_{3}$ ), -0.79 (dd, ${ }^{1} J=3.5 \mathrm{~Hz}, 1 \mathrm{H}$, $\left.\mathrm{YCH}_{2}\right),-0.97\left(\mathrm{dd},{ }^{1} J=3.5 \mathrm{~Hz}, 1 \mathrm{H}, \mathrm{YCH}_{2}\right) .{ }^{13} \mathrm{C} \mathrm{NMR}\left(126 \mathrm{MHz}, \mathrm{C}_{6} \mathrm{D}_{6}\right) \delta=134.4,131.8,128.0$, $123.4,122.1,120.8,120.7,104.2,100.0,70.3,53.5,35.5,29.8,24.3,5.9,4.3,4.0 .{ }^{29} \mathrm{Si}$ NMR $\left(99 \mathrm{MHz}, \mathrm{C}_{6} \mathrm{D}_{6}\right) \delta=-3.36,-24.34$. 
(4.40 mmol, 1 eq.) is dissolved in $100 \mathrm{~mL}$ pentane and $472 \mathrm{mg}$ 2,6-lutidine (4.4 mmol, 1 eq.) in $20 \mathrm{~mL}$ pentane are added dropwise to the stirred solution. The mixture is stirred at room temperature for 30 minutes and volatile compounds are removed in vacuo. The residue is washed once with cold pentane and recrystallization from a saturated toluene-solution yields yellow crystals (1.78 g, $4.0 \mathrm{mmol}, 91 \%) .{ }^{1} \mathrm{H} \mathrm{NMR}\left(400 \mathrm{MHz}, \mathrm{C}_{6} \mathrm{D}_{6}\right) \delta=6.76\left(\mathrm{dd},{ }^{3} J=8.1 \mathrm{~Hz}\right.$, $\left.{ }^{3} J=7.7 \mathrm{~Hz}, 1 \mathrm{H}, \mathrm{H}_{\mathrm{ar}}\right), 6.46\left(\mathrm{~d},{ }^{3} J=8.1 \mathrm{~Hz}, 1 \mathrm{H}, \mathrm{H}_{\mathrm{ar}}\right), 5.98\left(\mathrm{~d},{ }^{3} J=7.7 \mathrm{~Hz}, 1 \mathrm{H}, \mathrm{H}_{\mathrm{ar}}\right), 2.52(\mathrm{~s}, 3 \mathrm{H}$, $\left.\mathrm{CH}_{3}\right), 2.33$ (s, 3H, $\left.\mathrm{CH}_{3}\right), 2.15$ (s, 3H, $\left.\mathrm{CH}_{3}\right), 2.02$ (s, 3H, $\left.\mathrm{CH}_{3}\right), 1.85$ (s, 3H, $\left.\mathrm{CH}_{3}\right), 1.41$ (s, 2H, $\left.\mathrm{YCH}_{2}\right), 1.00$ (s, 9H, NC(CH3) $), 0.83$ (s, 3H, SiMe $), 0.74$ (s, 3H, SiMe 2$) .{ }^{13} \mathrm{C} \mathrm{NMR}(126 \mathrm{MHz}$, $\left.\mathrm{C}_{6} \mathrm{D}_{6}\right) \delta=172.2,155.0,138.7,127.2,126.6,122.8,122.0,114.3,106.7,54.4,41.3,33.7,26.0$, $15.6,14.1,11.4,11.0,8.3,7.4 .{ }^{29} \mathrm{Si} \mathrm{NMR}\left(99 \mathrm{MHz}, \mathrm{C}_{6} \mathrm{D}_{6}\right) \delta=-25.37$. elemental analysis calcd (\%) for $\mathrm{C}_{22} \mathrm{H}_{35} \mathrm{~N}_{2} \mathrm{SiY}$ : C, 59.44; H, 7.94; N, 6.30. Found: C, 59.66; H, 8.20; N, 6.22.

(C5 $\left.\mathrm{Me}_{4}\right) \mathrm{CH}_{2} \mathrm{CH}_{2} \mathrm{~N} t$ BuY(2,6-lutidinyl) (2) $20.0 \mathrm{mg} \quad\left(\mathrm{C}_{5} \mathrm{Me}_{4}\right) \mathrm{CH}_{2} \mathrm{CH}_{2} \mathrm{~N} t \mathrm{BuYCH}_{2} \mathrm{TMS}$ (thf) (42.8 $\mu \mathrm{mol}, 1$ eq.) is dissolved in $0.6 \mathrm{~mL} \mathrm{C}_{6} \mathrm{D}_{6}$ and $4.58 \mathrm{mg} 2,6$-lutidine ( $42.8 \mu \mathrm{mol}, 1$ eq.) are added dropwise to the stirred solution. The mixture is stirred at room temperature for 30 minutes and NMR analysis shows quantitative conversion. ${ }^{1} \mathrm{H}$ NMR $\left(400 \mathrm{MHz}, \mathrm{C}_{6} \mathrm{D}_{6}\right) \delta=6.79-6.72$ (m, 1H, $\left.\mathrm{H}_{\mathrm{ar}}\right), 6.45-6.39\left(\mathrm{~m}, 1 \mathrm{H}, \mathrm{H}_{\mathrm{ar}}\right), 6.01\left(\mathrm{~d}, 1 \mathrm{H}, \mathrm{H}_{\mathrm{ar}}\right), 3.61-3.57\left(\mathrm{~m}, 2 \mathrm{H}, \mathrm{CH}_{2} \mathrm{CH}_{2} \mathrm{~N}\right), 3.11-3.06$ (m, 2H, $\left.\mathrm{CH}_{2} \mathrm{CH}_{2} \mathrm{~N}\right), 2.45$ (s, 3H, $\left.\mathrm{CH}_{3}\right), 2.35$ (s, 2H, YCH$), 2.34$ (s, 3H, CH $\left.\mathrm{YH}_{3}\right), 2.20$ (s, 3H, $\left.\mathrm{CH}_{3}\right), 2.01\left(\mathrm{~s}, 3 \mathrm{H}, \mathrm{CH}_{3}\right), 1.11\left(\mathrm{~s}, 9 \mathrm{H}, \mathrm{NC}\left(\mathrm{CH}_{3}\right)_{3}\right)$.

(C9H6)SiMe2N $t$ BuY(2,6-lutidinyl) (3) $20.0 \mathrm{mg}\left(\mathrm{C}_{9} \mathrm{H}_{6}\right) \mathrm{SiMe}_{2} \mathrm{~N} t \mathrm{BuYCH}_{2} \mathrm{TMS}$ (thf) $(39.5 \mu \mathrm{mol}$, 1 eq.) is dissolved in $0.6 \mathrm{~mL} \mathrm{C}_{6} \mathrm{D}_{6}$ and $4.23 \mathrm{mg}$ 2,6-lutidine (39.5 $\mu \mathrm{mol}, 1$ eq.) are added dropwise to the stirred solution. The mixture is stirred at room temperature for 30 minutes and NMR analysis shows quantitative conversion. Diffusion crystallization in $\mathrm{C}_{6} \mathrm{D}_{6} /$ pentane at $30{ }^{\circ} \mathrm{C}$ yields yellow crystals. ${ }^{1} \mathrm{H}$ NMR $\left(400 \mathrm{MHz}, \mathrm{C}_{6} \mathrm{D}_{6}\right) \delta=8.08-8.03$ (m, $\left.1 \mathrm{H}, \mathrm{H}_{\text {Ind }}\right), 7.54-7.49$ ( $\mathrm{m}, 1 \mathrm{H}, \mathrm{H}_{\text {Ind }}$ ), 7.09-7.03 (m, 1H, $\mathrm{H}_{\text {Ind }}$ ), 6.82-6.67 (m, 4H, $\mathrm{H}_{\text {Ind }}, \mathrm{H}_{\mathrm{ar}}$ ), 6.18-6.14 (m, $1 \mathrm{H}, \mathrm{H}_{\mathrm{ar}}$ ), 6.07-6.01 (m, 1H, Har), 2.41 (s, 6H, $\left.\mathrm{CH}_{3}\right), 1.15$ (s, 9H, NC(CH3) $\left.)_{3}\right), 1.00$ (s, 3H, SiMe 2$), 0.88$ (s, $\left.3 \mathrm{H}, \mathrm{SiMe}_{2}\right), 0.29$ (s, 2H, YCH$) .{ }^{13} \mathrm{C} \mathrm{NMR}\left(126 \mathrm{MHz}, \mathrm{C}_{6} \mathrm{D}_{6}\right) \delta=169.0,164.7,156.3,136.3$, 133.6, 131.6, 126.5, 124.4, 122.0, 120.6, 120.1, 115.7, 104.0, 54.2, 43.1, 34.5, 24.7, 6.4, 4.3. ${ }^{29} \mathrm{Si}$ NMR (99 MHz, $\left.\mathrm{C}_{6} \mathrm{D}_{6}\right) \delta=-24.65$. elemental analysis calcd $(\%)$ for C22H29YN2Si: C, 60.26; H, 6.67; N, 6.39. Found: C, 59.92; H, 7.04; N, 7.09.

(C5 $\left.\mathrm{Ce}_{4}\right) \mathrm{SiMe}_{2} \mathrm{NPhY}$ (2,6-lutidinyl) (4) $\quad 20.0 \mathrm{mg} \quad\left(\mathrm{C}_{5} \mathrm{Me}_{4}\right) \mathrm{Me}_{2} \mathrm{SiNPhYCH}_{2} \mathrm{TMS}(\mathrm{thf})_{2}$ (33.9 $\mu \mathrm{mol}, 1$ eq.) is dissolved in $0.6 \mathrm{~mL} \mathrm{C}_{6} \mathrm{D}_{6}$ and $3.63 \mathrm{mg}$ 2,6-lutidine (33.9 $\mu \mathrm{mol}, 1$ eq.) are added dropwise to the stirred solution. The mixture is stirred at room temperature for 30 minutes 
and NMR analysis shows quantitative conversion. ${ }^{1} \mathrm{H}$ NMR $\left(400 \mathrm{MHz}, \mathrm{C}_{6} \mathrm{D}_{6}\right) \delta=7.29-7.17$ (m, 2H, Ph-H), 6.72 (t, 2H, Ph-H), 6.55 (d, 1H, Ph-H), 6.38 (s, 1H, $\mathrm{H}_{\mathrm{ar}}$ ), 6.29-6.22 (m, 1H, $\mathrm{H}_{\mathrm{ar}}$ ), $5.69\left(\mathrm{~s}, 1 \mathrm{H}, \mathrm{H}_{\mathrm{ar}}\right), 2.17\left(\mathrm{~s}, 6 \mathrm{H}, \mathrm{CH}_{3}\right), 2.12\left(\mathrm{~s}, 3 \mathrm{H}, \mathrm{CH}_{3}\right), 2.10\left(\mathrm{~s}, 2 \mathrm{H}, \mathrm{YCH}_{2}\right), 2.04\left(\mathrm{~s}, 3 \mathrm{H}, \mathrm{CH}_{3}\right)$, 1.87 (s, 3H, $\left.\mathrm{CH}_{3}\right), 0.97$ (s, 6H, $\mathrm{SiMe}_{2}$ ). ${ }^{13} \mathrm{C} \mathrm{NMR}\left(126 \mathrm{MHz}, \mathrm{C}_{6} \mathrm{D}_{6}\right) \delta=165.9,156.5,155.4$, 147.1, 137.5, 129.2, 129.0, 128.2, 126.7, 125.3, 123.0, 119.4, 115.7, 115.2, 110.9, 106.9, 48.3, 31.6, 22.9, 14.0, 13.5, 11.1, 4.5. ${ }^{29} \mathrm{Si}$ NMR (99 MHz, $\left.\mathrm{C}_{6} \mathrm{D}_{6}\right) \delta=-22.49$.

$\left(\mathrm{C}_{5} \mathrm{Me}_{4}\right) \mathrm{CH}_{2} \mathrm{CH}_{2} \mathrm{~N} t \mathrm{BuMg} \cdot 2$ THF $5.00 \mathrm{~g}\left(\mathrm{C}_{5} \mathrm{Me}_{4} \mathrm{H}\right) \mathrm{CH}_{2} \mathrm{CH}_{2} \mathrm{NH} t \mathrm{Bu}$ (22.6 mmol, 1.0 eq.) are dissolved in $100 \mathrm{ml}$ toluene and $5 \mathrm{~mL}$ THF. The solution is cooled to $0{ }^{\circ} \mathrm{C}$ and $23.0 \mathrm{~mL}$ Di$n$ butylmagnesium (1.0 $\mathrm{M}$ in heptane, $3.14 \mathrm{~g}, 23.0 \mathrm{mmol}, 1.0 \mathrm{eq}$.) is added within 30 minutes. After stirring the solution over night at r.t. the solvents are evaporated under high vacuum at $60{ }^{\circ} \mathrm{C}$. Crystallization at $-80{ }^{\circ} \mathrm{C}$ in THF yields a green solid $(4.31 \mathrm{~g}, 17.7 \mathrm{mmol}, 78 \%) .{ }^{1} \mathrm{H}$ NMR (400 MHz, $\left.\mathrm{C}_{6} \mathrm{D}_{6}\right) \delta=3.54(\mathrm{~m}, 8 \mathrm{H}, \mathrm{THF}-\mathrm{H}), 3.15\left(\mathrm{~m}, 1 \mathrm{H}, \mathrm{CH}_{2} \mathrm{CH}_{2} \mathrm{~N}\right), 2.70\left(\mathrm{~m}, 1 \mathrm{H}, \mathrm{CH}_{2} \mathrm{CH}_{2} \mathrm{~N}\right)$, $2.65\left(\mathrm{~m}, 1 \mathrm{H}, \mathrm{CH}_{2} \mathrm{CH}_{2} \mathrm{~N}\right), 2.48\left(\mathrm{~m}, 1 \mathrm{H}, \mathrm{CH}_{2} \mathrm{CH}_{2} \mathrm{~N}\right), 2.24\left(\mathrm{~s}, 3 \mathrm{H}, \mathrm{CH}_{3}\right), 2.12\left(\mathrm{~s}, 3 \mathrm{H}, \mathrm{CH}_{3}\right), 2.06$ (s, 3H, $\left.\mathrm{CH}_{3}\right), 2.04\left(\mathrm{~s}, 3 \mathrm{H}, \mathrm{CH}_{3}\right), 1.33-1.49$ (m, 8H, THF-H), $0.88\left(\mathrm{~s}, 9 \mathrm{H}, \mathrm{NC}\left(\mathrm{CH}_{3}\right)_{3}\right) .{ }^{13} \mathrm{C} \mathrm{NMR}$ $\left(126 \mathrm{MHz}, \mathrm{C}_{6} \mathrm{D}_{6}\right) \delta=116.2,111.9,110.9,109.7,106.7,68.3,52.2,50.3,34.1,30.1,25.7,13.0$, $12.2,11.4,11.1$.

(C5Me) $\mathrm{CiMe}_{2} \mathrm{~N} t \mathrm{BuMg}$ - 2 THF $4.00 \mathrm{~g}\left(\mathrm{C}_{5} \mathrm{Me}_{4} \mathrm{H}\right) \mathrm{SiMe}_{2} \mathrm{NH} t \mathrm{Bu}$ (15.9 mmol, 1.0 eq.) are dissolved in $100 \mathrm{ml}$ toluene and $5 \mathrm{~mL}$ THF. The solution is cooled to $0{ }^{\circ} \mathrm{C}$ and $16.2 \mathrm{~mL} \mathrm{Di}$ nbutylmagnesium (1.0 $\mathrm{M}$ in heptane, $2.25 \mathrm{~g}, 16.2 \mathrm{mmol}, 1.0 \mathrm{eq}$.) is added within 30 minutes. After stirring the solution over night at r.t. the solvents are evaporated under high vacuum at $60{ }^{\circ} \mathrm{C}$. Crystallization at $-80{ }^{\circ} \mathrm{C}$ in $\mathrm{THF}$ yields an orange solid $(3.86 \mathrm{~g}, 11.2 \mathrm{mmol}, 70 \%) .{ }^{1} \mathrm{H}$ NMR (400 MHz, $\left.\mathrm{C}_{6} \mathrm{D}_{6}\right) \delta=3.25-3.48(\mathrm{~m}, 8 \mathrm{H}, \mathrm{THF}-\mathrm{H}), 2.52\left(\mathrm{~s}, 6 \mathrm{H}, \mathrm{CH}_{3}\right), 2.19\left(\mathrm{~s}, 6 \mathrm{H}, \mathrm{CH}_{3}\right)$, 1.42 (s, 9H, $\left.\mathrm{NC}\left(\mathrm{CH}_{3}\right)_{3}\right), 1.17-1.29$ (m, 8H, THF-H), 0.98 (s, 6H, SiMe $) .{ }^{13} \mathrm{C} \mathrm{NMR}(126 \mathrm{MHz}$, $\left.\mathrm{C}_{6} \mathrm{D}_{6}\right) \delta=116.0,114.2,108.4,69.1,50.8,37.6,24.8,14.7,12.0,8.6$.

(C9H6)SiMe2 $\mathrm{N} t$ BuMg • 2 THF $5.00 \mathrm{~g}\left(\mathrm{C}_{9} \mathrm{H}_{7}\right) \mathrm{SiMe}_{2} \mathrm{NH} t \mathrm{Bu}$ (20.4 mmol, 1.0 eq.) are dissolved in $60 \mathrm{ml}$ toluene and $5 \mathrm{~mL}$ THF. The solution is cooled to $0^{\circ} \mathrm{C}$ and $21.4 \mathrm{~mL} \mathrm{Di-}$ nbutylmagnesium (1.0 M in heptane, $2.96 \mathrm{~g}, 21.4 \mathrm{mmol}, 1.0 \mathrm{eq}$.) is added within 30 minutes. After stirring the solution over night at r.t. the solvents are evaporated under high vacuum at $60{ }^{\circ} \mathrm{C}$. Crystallization at $-80{ }^{\circ} \mathrm{C}$ in THF yields a yellowish solid $(4.38 \mathrm{~g}, 16.4 \mathrm{mmol}, 70 \%) .{ }^{1} \mathrm{H}$ NMR (400 MHz, $\left.\mathrm{C}_{6} \mathrm{D}_{6}\right) \delta=8.18\left(\mathrm{~d},{ }^{3} J=7.9 \mathrm{~Hz}, 1 \mathrm{H}, \mathrm{H}_{\mathrm{ar}}\right), 8.01\left(\mathrm{~d},{ }^{3} J=7.9 \mathrm{~Hz}, 1 \mathrm{H}, \mathrm{H}_{\mathrm{ar}}\right), 7.31$ $\left(\mathrm{d},{ }^{3} J=3.4 \mathrm{~Hz}, 1 \mathrm{H}, \mathrm{H}_{\mathrm{ar}}\right), 7.14\left(\mathrm{dd},{ }^{3} J=7.9 \mathrm{~Hz},{ }^{3} J=6.7 \mathrm{~Hz}, 1 \mathrm{H}, \mathrm{H}_{\mathrm{ar}}\right), 7.07\left(\mathrm{dd},{ }^{3} J=7.9 \mathrm{~Hz},{ }^{3} J\right.$ $\left.=6.7 \mathrm{~Hz}, 1 \mathrm{H}, \mathrm{H}_{\mathrm{ar}}\right), 6.89\left(\mathrm{~d},{ }^{3} \mathrm{~J}=3.5 \mathrm{~Hz}, 1 \mathrm{H}, \mathrm{H}_{\mathrm{ar}}\right), 3.15(\mathrm{~s}, 8 \mathrm{H}, \mathrm{THF}-\mathrm{H}), 1.46\left(\mathrm{~s}, 9 \mathrm{H}, \mathrm{NC}\left(\mathrm{CH}_{3}\right)_{3}\right)$, 
1.12 (s, 8H, THF-H), 1.04 (s, 3H, SiMe $), 0.91$ (s, 3H, SiMe 2$) .{ }^{13} \mathrm{C} \mathrm{NMR}\left(126 \mathrm{MHz}, \mathrm{C}_{6} \mathrm{D}_{6}\right) \delta=$ 135.9, 135.1, 124.1, 120.8, 120.5, 116.7, 116.1, 103.5, 95.4, 69.7, 51.1., 38.1, 25.1, 6.6, 6.2.

(2,6-lutidinyl))AlCl 2 - THF $10.8 \mathrm{~mL}$ 2,6-lutidine (10.0 g, $93.3 \mathrm{mmol}, 1.0 \mathrm{eq}$.) are dissolved in $100 \mathrm{~mL}$ THF and cooled to $-78^{\circ} \mathrm{C}$. After slow addition of $39.2 \mathrm{~mL} n \mathrm{BuLi}$ ( $2.5 \mathrm{M}$ in pentane, $6.28 \mathrm{~g}, 1.05$ eq.) a solution of $12.4 \mathrm{~g}$ aluminumchlorid (93.3 mmol, 1.0 eq.) in $300 \mathrm{~mL}$ THF is added within an hour. The mixture is carefully allowed to heat up to r.t. and the solvents are evaporated under reduced pressure. The residue is washed with $200 \mathrm{~mL}$ pentane and is suspended in $400 \mathrm{~mL}$ toluene after drying. The solvent of the filtrate is removed under vacuum. Crystallization at $-90{ }^{\circ} \mathrm{C}$ in THF yields an off-white solid $(18.8 \mathrm{~g}, 68.1 \mathrm{mmol}, 73 \%) .{ }^{1} \mathrm{H}$ NMR $\left(400 \mathrm{MHz}, \mathrm{C}_{6} \mathrm{D}_{6}\right) \delta=6.91\left(\mathrm{dd},{ }^{3} J=7.6 \mathrm{~Hz},{ }^{3} J=7.7 \mathrm{~Hz}, 1 \mathrm{H}, \mathrm{H}_{\mathrm{ar}}\right), 6.76\left(\mathrm{~d},{ }^{3} J=7.7 \mathrm{~Hz}, 1 \mathrm{H}, \mathrm{H}_{\mathrm{ar}}\right)$, $6.32\left(\mathrm{~d},{ }^{3} \mathrm{~J}=7.6 \mathrm{~Hz}, 1 \mathrm{H}, \mathrm{H}_{\mathrm{ar}}\right), 3.78-3.91$ (m, 4H, THF-H), 2.58 (s, 3H, CH3), 1.97 (s, 2H, $\left.\mathrm{AlCH}_{2}\right), 1.13$ - 1.25 (m, 4H, THF-H). ${ }^{13} \mathrm{C}$ NMR (126 MHz, C $\left.6 \mathrm{D}_{6}\right) \delta=164.2,155.1,139.2$, 121.7, 121.3, 70.4, 25.1, 21.0, 16.3. ${ }^{27} \mathrm{Al} \mathrm{NMR}\left(78 \mathrm{MHz}, \mathrm{C}_{6} \mathrm{D}_{6}\right) \delta=81.5$.

(C5Me4)SiMe 2 N $t$ BuAl(2,6-lutidinyl) (5) 5.62 g $\left(\mathrm{C}_{5} \mathrm{Me}_{4}\right) \mathrm{SiMe}_{2} \mathrm{~N} t \mathrm{BuMg} \cdot 2 \mathrm{THF}$ (16.3 mmol, 1.0 eq.) are dissolved in $150 \mathrm{~mL}$ THF and cooled to $-10{ }^{\circ} \mathrm{C}$. A solution of $4.49 \mathrm{~g}(2,6-$ lutidinyl $) \mathrm{AlCl}_{2}$. THF (16.3 mmol, 1.0 eq.) in $20 \mathrm{~mL} \mathrm{THF} \mathrm{is} \mathrm{added} \mathrm{within} \mathrm{an} \mathrm{hour.} \mathrm{The} \mathrm{mixture}$ is stirred over night at r.t. and the solvent is evaporated under reduced pressure. The residue is suspended in $200 \mathrm{~mL}$ pentane and the solvent of the filtrate is removed. Orange crystals could be received by recrystallization in pentane at $-60{ }^{\circ} \mathrm{C}(5.14 \mathrm{~g}, 13.4 \mathrm{mmol}, 83 \%) .{ }^{1} \mathrm{H}$ NMR (400 $\left.\mathrm{MHz}, \mathrm{C}_{6} \mathrm{D}_{6}\right) \delta=6.67\left(\mathrm{dd},{ }^{3} J=7.7 \mathrm{~Hz},{ }^{3} J=7.7 \mathrm{~Hz}, 1 \mathrm{H}, \mathrm{H}_{\mathrm{ar}}\right), 6.50\left(\mathrm{~d},{ }^{3} J=7.7 \mathrm{~Hz}, 1 \mathrm{H}, \mathrm{H}_{\mathrm{ar}}\right), 6.15$ $\left(\mathrm{d},{ }^{3} \mathrm{~J}=7.7 \mathrm{~Hz}, 1 \mathrm{H}, \mathrm{H}_{\mathrm{ar}}\right), 2.40\left(\mathrm{~s}, 6 \mathrm{H}, \mathrm{CH}_{3}\right), 2.21\left(\mathrm{~s}, 3 \mathrm{H}, \mathrm{CH}_{3}\right), 2.14-1.70\left(\mathrm{~m}, 6 \mathrm{H}, \mathrm{CH}_{3}\right), 1.32$ -1.59 (m, 2H, $\left.\mathrm{AlCH}_{2}\right), 1.25$ (s, 9H, NC(CH3) $\left.)_{3}\right), 0.81$ (s, 6H, $\left.\mathrm{SiMe}_{2}\right) .{ }^{13} \mathrm{C} \mathrm{NMR}(126 \mathrm{MHz}$, $\left.\mathrm{C}_{6} \mathrm{D}_{6}\right) \delta=168.4,154.3,139.7,122.7,121.0,108.3,51.4,35.7,22.8,19.1,15.3,11.6,6.6 .{ }^{27} \mathrm{Al}$ NMR $\left(78 \mathrm{MHz}, \mathrm{C}_{6} \mathrm{D}_{6}\right) \delta=65.1 .{ }^{29} \mathrm{Si} \mathrm{NMR}\left(60 \mathrm{MHz}, \mathrm{C}_{6} \mathrm{D}_{6}\right) \delta=-14.6$. elemental analysis calcd (\%) for C22H35AlN2Si: C, 69.06; H, 9.22; N, 7.32. Found: C, 69.28; H, 9.37; N, 7.10. 


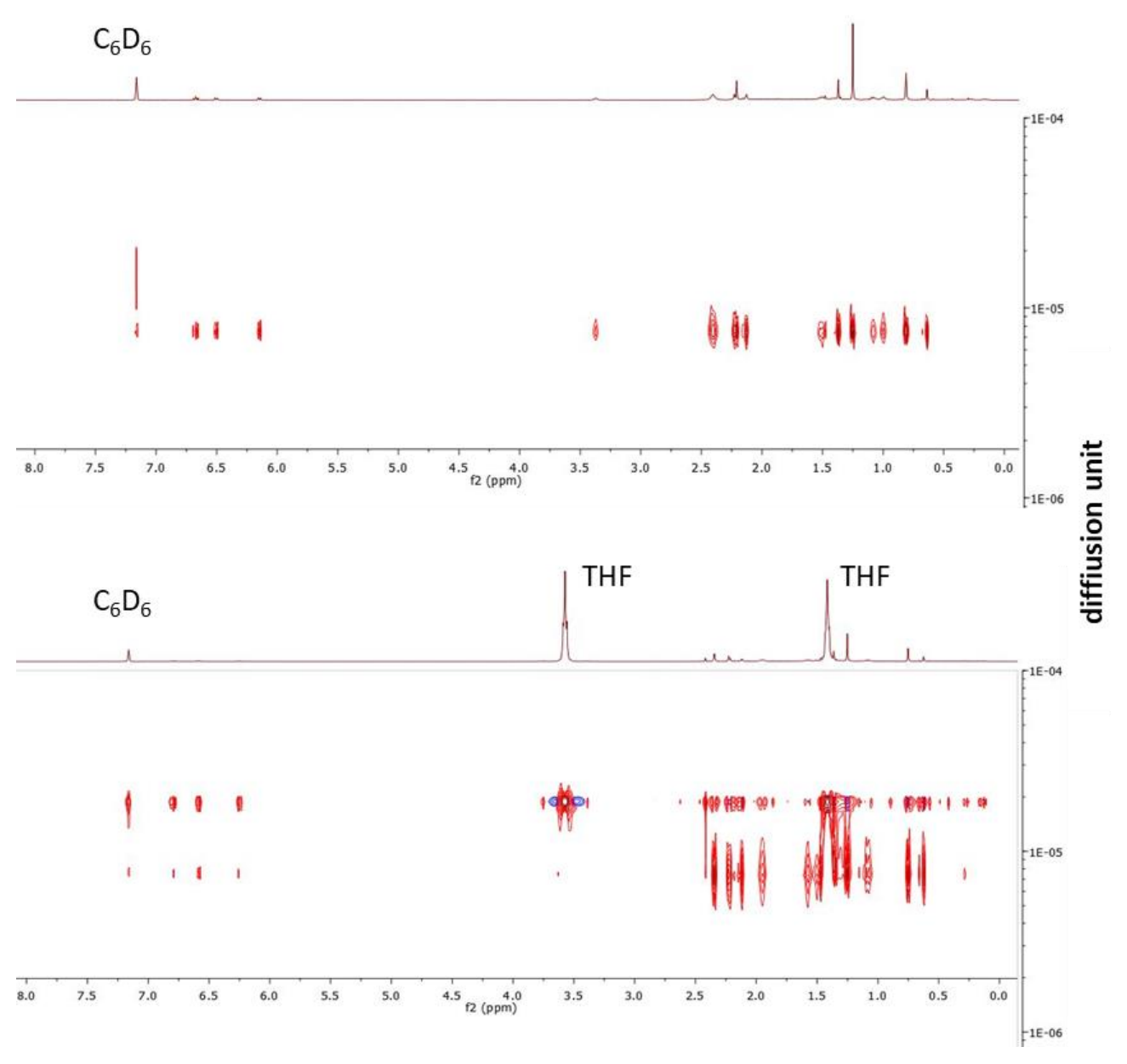

Figure S1. ${ }^{1} \mathrm{H}$ DOSY NMR spectra of 5 in $\mathrm{C}_{6} \mathrm{D}_{6}$ without (top) and with 10 eq. THF (bottom).

( $\left.\mathrm{C}_{5} \mathrm{Me}_{4}\right) \mathrm{CH}_{2} \mathrm{CH}_{2} \mathrm{~N} t \mathrm{BuAl}(2,6-l u t i d i n y l)(6) \quad 1.25 \mathrm{~g} \quad\left(\mathrm{C}_{5} \mathrm{Me}_{4}\right) \mathrm{CH}_{2} \mathrm{CH}_{2} \mathrm{~N} t \mathrm{BuMg} \cdot 2 \mathrm{THF}$ (5.13 mmol, 1.0 Äq.) are dissolved in $50 \mathrm{~mL}$ THF and cooled to $0{ }^{\circ} \mathrm{C}$. A solution of $1.42 \mathrm{~g}(2,6-$ lutidinyl) $\mathrm{AlCl}_{2}$. THF (5.13 mmol, 1.0 eq.) in $20 \mathrm{~mL}$ THF is added within 30 minutes. The mixture is stirred over night at r.t. and the solvent is evaporated under reduced pressure. The residue is suspended in $50 \mathrm{~mL}$ pentane and the solvent of the filtrate is removed. The red-brown solid could be received by precipitation in pentane $-60{ }^{\circ} \mathrm{C}(1.02 \mathrm{~g}, 2.89 \mathrm{mmol}, 56 \%) .{ }^{1} \mathrm{H} \mathrm{NMR}$ $\left(400 \mathrm{MHz}, \mathrm{C}_{6} \mathrm{D}_{6}\right) \delta=6.73\left(\mathrm{dd},{ }^{3} J=7.5 \mathrm{~Hz},{ }^{3} J=7.6 \mathrm{~Hz}, 1 \mathrm{H}, \mathrm{H}_{\mathrm{ar}}\right), 6.60\left(\mathrm{~d},{ }^{3} J=7.6 \mathrm{~Hz}, 1 \mathrm{H}, \mathrm{H}_{\mathrm{ar}}\right)$, $6.20\left(\mathrm{~d},{ }^{3} J=7.5 \mathrm{~Hz}, 1 \mathrm{H}, \mathrm{H}_{\mathrm{ar}}\right), 3.46\left(\mathrm{t},{ }^{3} \mathrm{~J}=6.6 \mathrm{~Hz}, 2 \mathrm{H}, \mathrm{CH}_{2} \mathrm{CH}_{2} \mathrm{~N}\right), 3.05\left(\mathrm{t},{ }^{3} J=6.6 \mathrm{~Hz}, 2 \mathrm{H}\right.$, $\left.\mathrm{CH}_{2} \mathrm{CH}_{2} \mathrm{~N}\right), 2.21\left(\mathrm{~s}, 3 \mathrm{H}, \mathrm{CH}_{3}\right), 2.16\left(\mathrm{~s}, 6 \mathrm{H}, \mathrm{CH}_{3}\right), 2.14-1.88\left(\mathrm{~m}, 6 \mathrm{H}, \mathrm{CH}_{3}\right), 1.40\left(\mathrm{~s}, 2 \mathrm{H}, \mathrm{AlCH}_{2}\right)$, $1.15\left(\mathrm{~s}, 9 \mathrm{H}, \mathrm{NC}\left(\mathrm{CH}_{3}\right)_{3}\right) .{ }^{13} \mathrm{C} \mathrm{NMR}\left(126 \mathrm{MHz}, \mathrm{C}_{6} \mathrm{D}_{6}\right) \delta=168.3,153.7,139.1,129.3,124.9$, 122.6, 120.8, 105.6, 51.7, 45.0, 31.1, 27.1, 22.6, 11.9, 11.8, 11.2.

(C9H6)SiMe2N $t$ BuAl(2,6-lutidinyl) (7) $3.00 \mathrm{~g} \quad\left(\mathrm{C}_{9} \mathrm{H}_{6}\right) \mathrm{SiMe}_{2} \mathrm{~N} t \mathrm{BuMg} \cdot 2 \mathrm{THF} \quad(11.2 \mathrm{mmol}$, 1.0 Äq.) are dissolved in $150 \mathrm{~mL}$ toluene and cooled to $0{ }^{\circ} \mathrm{C}$. A solution of $3.09 \mathrm{~g}(2,6-$ lutidinyl) $) \mathrm{AlCl}_{2}$ - THF (11.2 mmol, 1.0 eq.) in $50 \mathrm{~mL}$ toluene is added within an hour. The mixture is stirred over night at r.t. and the solvent is evaporated under reduced pressure. The 
residue is dissolved in $50 \mathrm{~mL}$ pentane and after one week at $-45^{\circ} \mathrm{C}$, the solid is separated and dried. Yellow crystals could be received by recrystallization in toluene at $-45^{\circ} \mathrm{C}(3.29 \mathrm{~g}$, $8.74 \mathrm{mmol}, 78 \%) .{ }^{1} \mathrm{H}$ NMR (400 MHz, $\left.\mathrm{C}_{6} \mathrm{D}_{6}\right) \delta=7.62$ (d, 1H, $\left.\mathrm{H}_{\text {Ind }}\right), 7.09-7.16$ (m, 1H, $\mathrm{H}_{\text {Ind }}$ ), $6.82-6.88$ (m, 2H, $\left.\mathrm{H}_{\text {Ind }}\right), 6.67-6.76\left(\mathrm{~m}, 2 \mathrm{H}, \mathrm{H}_{\mathrm{ar}}\right), 6.49\left(\mathrm{~d},{ }^{3} J=4.7 \mathrm{~Hz}, 1 \mathrm{H}, \mathrm{H}_{\text {Ind }}\right), 6.37$ (d, ${ }^{3} J$ $\left.=4.7 \mathrm{~Hz}, 1 \mathrm{H}, \mathrm{H}_{\text {Ind }}\right), 6.18\left(\mathrm{~d}, 1 \mathrm{H}, \mathrm{H}_{\mathrm{ar}}\right), 2.86\left(\mathrm{~s}, 6 \mathrm{H}, \mathrm{CH}_{3}\right), 1.31\left(\mathrm{~s}, 9 \mathrm{H}, \mathrm{NC}\left(\mathrm{CH}_{3}\right)_{3}\right), 1.28\left(\mathrm{~d},{ }^{2} \mathrm{~J}=\right.$ $12.0 \mathrm{~Hz}, 1 \mathrm{H}, \mathrm{AlCH}_{2}$ ), 0.43 (s, 3H, SiMe $), 0.33$ (s, 3H, SiMe $), 1.04$ (d, ${ }^{2} J=12.0 \mathrm{~Hz}, 1 \mathrm{H}$, $\left.\mathrm{AlCH}_{2}\right) .{ }^{13} \mathrm{C} \mathrm{NMR}\left(126 \mathrm{MHz}, \mathrm{C}_{6} \mathrm{D}_{6}\right) \delta=169.2,155.1,146.1,143.0,138.7,136.8,122.9,120.9$, 120.6, 120.4, 120.3, 119.0, 116.3, 110.4, 51.3, 35.5, 28.3, 24.7, 6.6, 4.6. elemental analysis calcd (\%) for C22H29AlN2Si: C, 70.17; H, 7.76; N, 7.44. Found: C, 69.92; H, 8.04; N, 7.09. 


\section{Single Crystal X-Ray Diffraction Experiments}

\section{SC-XRD determination of compound 1 (CCDC 1936317)}

A yellow fragment-like specimen of $\mathrm{C}_{26} \mathrm{H}_{43} \mathrm{~N}_{2} \mathrm{OSiY}$, approximate dimensions $0.139 \mathrm{~mm} \mathrm{x}$ $0.148 \mathrm{~mm} \times 0.156 \mathrm{~mm}$, was used for the X-ray crystallographic analysis. The X-ray intensity data were measured on a Bruker D8 Venture system equipped with a Helios optic monochromator and a Mo TXS rotating anode $(\lambda=0.71073 \AA)$.

A total of 1713 frames were collected. The total exposure time was 4.31 hours. The frames were integrated with the Bruker SAINT software package using a narrow-frame algorithm. The integration of the data using a monoclinic unit cell yielded a total of 60076 reflections to a maximum $\theta$ angle of $25.35^{\circ}$ ( $0.83 \AA$ resolution), of which 4876 were independent (average redundancy 12.321 , completeness $\left.=100.0 \%, \mathrm{R}_{\text {int }}=6.84 \%, \mathrm{R}_{\text {sig }}=2.79 \%\right)$ and $3942(80.84 \%)$ were greater than $2 \sigma\left(F^{2}\right)$. The final cell constants of $\underline{a}=10.9805(4) \AA, \underline{b}=20.9144(8) \AA$, $\underline{c}=$ 11.6587(4) $\AA, \beta=94.721(2)^{\circ}$, volume $=2668.34(17) \AA^{3}$, are based upon the refinement of the XYZ-centroids of 132 reflections above $20 \sigma(\mathrm{I})$ with $4.202^{\circ}<2 \theta<34.37^{\circ}$. Data were corrected for absorption effects using the Multi-Scan method (SADABS). The ratio of minimum to maximum apparent transmission was 0.866. The calculated minimum and maximum transmission coefficients (based on crystal size) are 0.7200 and 0.7450 .

The final anisotropic full-matrix least-squares refinement on $\mathrm{F}^{2}$ with 290 variables converged at $\mathrm{R} 1=3.34 \%$, for the observed data and $\mathrm{wR} 2=8.15 \%$ for all data. The goodness-of-fit was 1.070. The largest peak in the final difference electron density synthesis was $0.712 \mathrm{e}^{-} / \AA^{3}$ and the largest hole was $-0.613 \mathrm{e}^{-} / \AA^{3}$ with an RMS deviation of $0.070 \mathrm{e}^{-} / \AA^{3}$. On the basis of the final model, the calculated density was $1.286 \mathrm{~g} / \mathrm{cm}^{3}$ and $\mathrm{F}(000), 1096 \mathrm{e}^{-}$.

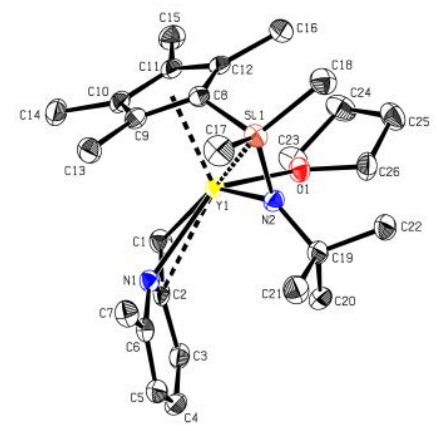

Figure S2. Ortep drawing with 50\% ellipsoids for compound $\mathbf{1 .}$ 
Table S1. Sample and crystal data for compound $\mathbf{1 .}$

$\begin{array}{lll}\text { Identification code } & \mathrm{PahPh} 7 \mathrm{AP} 9248-100 & \\ \text { Chemical formula } & \mathrm{C}_{26} \mathrm{H}_{43} \mathrm{~N}_{2} \mathrm{OSiY} & \\ \text { Formula weight } & 516.62 & \\ \text { Temperature } & 100(2) \mathrm{K} & \\ \text { Wavelength } & 0.71073 \AA \\ \text { Crystal size } & 0.139 \times 0.148 \mathrm{x} 0.156 \mathrm{~mm} \\ \text { Crystal habit } & \text { yellow fragment } & \\ \text { Crystal system } & \text { monoclinic } & \\ \text { Space group } & \mathrm{P} 121 / \mathrm{n} 1 & \\ \text { Unit cell dimensions } & \mathrm{a}=10.9805(4) \AA & \alpha=90^{\circ} \\ & \mathrm{b}=20.9144(8) \AA & \beta=94.721(2)^{\circ} \\ & \mathrm{c}=11.6587(4) \AA & \gamma=90^{\circ} \\ \text { Volume } & 2668.34(17) \AA^{3} & \\ \text { Z } & 4 & \\ \text { Density (calculated) } & 1.286 \mathrm{~g} / \mathrm{cm}^{3} & \\ \text { Absorption coefficient } & 2.250 \mathrm{~mm}^{-1} & \\ \text { F(000) } & 1096 & \end{array}$

Table S2. Data collection and structure refinement for compound 1.

\begin{tabular}{|c|c|}
\hline Diffractometer & Bruker D8 Venture \\
\hline Radiation source & TXS rotating anode, Mo \\
\hline $\begin{array}{l}\text { Theta range for data } \\
\text { collection }\end{array}$ & 1.95 to $25.35^{\circ}$ \\
\hline Index ranges & $-13<=\mathrm{h}<=13,-25<=\mathrm{k}<=25,-14<=1<=14$ \\
\hline Reflections collected & 60076 \\
\hline Independent reflections & $4876[\mathrm{R}(\mathrm{int})=0.0684]$ \\
\hline $\begin{array}{l}\text { Coverage of independent } \\
\text { reflections }\end{array}$ & $100.0 \%$ \\
\hline Absorption correction & Multi-Scan \\
\hline Max. and min. transmission & 0.7450 and 0.7200 \\
\hline Refinement method & Full-matrix least-squares on $\mathrm{F}^{2}$ \\
\hline Refinement program & SHELXL-2014/7 (Sheldrick, 2014) \\
\hline Function minimized & $\Sigma \mathrm{w}\left(\mathrm{F}_{\mathrm{o}}^{2}-\mathrm{F}_{\mathrm{c}}^{2}\right)^{2}$ \\
\hline Data / restraints / parameters & 4876 / 0 / 290 \\
\hline Goodness-of-fit on $F^{2}$ & 1.070 \\
\hline$\Delta / \sigma_{\max }$ & 0.001 \\
\hline \multirow[t]{2}{*}{ Final $\mathbf{R}$ indices } & $\begin{array}{l}\mathrm{R} 1=0.0334, \mathrm{wR} 2= \\
0.0714\end{array}$ \\
\hline & $\begin{array}{l}\mathrm{R} 1=0.0523, \mathrm{wR} 2= \\
0.0815\end{array}$ \\
\hline Weighting scheme & $\begin{array}{l}\mathrm{w}=1 /\left[\sigma^{2}\left(\mathrm{~F}_{\mathrm{o}}^{2}\right)+(0.0234 \mathrm{P})^{2}+4.9294 \mathrm{P}\right] \\
\text { where } \mathrm{P}=\left(\mathrm{F}_{\mathrm{o}}^{2}+2 \mathrm{~F}_{\mathrm{c}}^{2}\right) / 3\end{array}$ \\
\hline
\end{tabular}


Largest diff. peak and hole $\quad 0.712$ and $-0.613 \mathrm{e}^{-3}$

R.M.S. deviation from mean $0.070 \mathrm{e}^{-3}$

\section{SC-XRD determination of compound 3 (CCDC 1936316)}

A clear colourless fragment-like specimen of $\mathrm{C}_{22} \mathrm{H}_{29} \mathrm{~N}_{2} \mathrm{SiY}$, approximate dimensions $0.126 \mathrm{~mm}$ x $0.178 \mathrm{~mm} \times 0.234 \mathrm{~mm}$, was used for the X-ray crystallographic analysis. The X-ray intensity data were measured on a Bruker D8 Venture Duo IMS system equipped with a Helios optic monochromator and a Mo IMS microsource $(\lambda=0.71073 \AA)$.

A total of 1563 frames were collected. The total exposure time was 0.82 hours. The frames were integrated with the Bruker SAINT software package using a narrow-frame algorithm. The integration of the data using a monoclinic unit cell yielded a total of 66552 reflections to a maximum $\theta$ angle of $29.57^{\circ}(0.72 \AA$ resolution), of which 5978 were independent (average redundancy 11.133 , completeness $\left.=100.0 \%, \mathrm{R}_{\text {int }}=9.34 \%, \mathrm{R}_{\text {sig }}=4.29 \%\right)$ and $4877(81.58 \%)$ were greater than $2 \sigma\left(\mathrm{F}^{2}\right)$. The final cell constants of $\underline{\mathrm{a}}=10.4940(5) \AA, \underline{\mathrm{b}}=12.6978(6) \AA$, $\underline{\mathrm{c}}=$ 15.9846(7) $\AA, \beta=91.044(2)^{\circ}$, volume $=2129.61(17) \AA^{3}$, are based upon the refinement of the XYZ-centroids of 9863 reflections above $20 \sigma(\mathrm{I})$ with $4.682^{\circ}<2 \theta<61.07^{\circ}$. Data were corrected for absorption effects using the Multi-Scan method (SADABS). The ratio of minimum to maximum apparent transmission was 0.793 . The calculated minimum and maximum transmission coefficients (based on crystal size) are 0.5600 and 0.7190 .

The final anisotropic full-matrix least-squares refinement on $\mathrm{F}^{2}$ with 249 variables converged at $\mathrm{R} 1=3.48 \%$, for the observed data and $\mathrm{wR} 2=6.92 \%$ for all data. The goodness-of-fit was 1.041. The largest peak in the final difference electron density synthesis was $0.461 \mathrm{e}^{-} / \AA^{3}$ and the largest hole was $-0.386 \mathrm{e}^{-} / \AA^{3}$ with an RMS deviation of $0.084 \mathrm{e}^{-} / \AA^{3}$. On the basis of the final model, the calculated density was $1.368 \mathrm{~g} / \mathrm{cm}^{3}$ and $\mathrm{F}(000), 912 \mathrm{e}^{-}$.

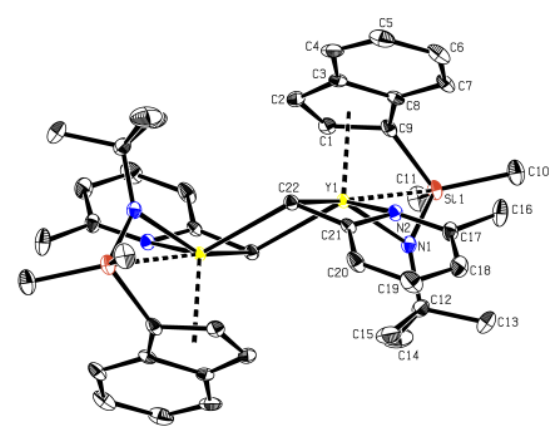

Figure S3. Ortep drawing with 50\% ellipsoids for compound 3. 
Table S3. Sample and crystal data for compound 3.

$\begin{array}{lll}\text { Identification code } & \text { PahPh6 AP8358-100 } \\ \text { Chemical formula } & \mathrm{C}_{22} \mathrm{H}_{29} \mathrm{~N}_{2} \mathrm{SiY} & \\ \text { Formula weight } & 438.47 & \\ \text { Temperature } & 100(2) \mathrm{K} & \\ \text { Wavelength } & 0.71073 \AA & \\ \text { Crystal size } & 0.126 \times 0.178 \times 0.234 \mathrm{~mm} \\ \text { Crystal habit } & \text { clear colourless fragment } \\ \text { Crystal system } & \text { monoclinic } & \\ \text { Space group } & \mathrm{P} 121 / \mathrm{n} 1 & \\ \text { Unit cell dimensions } & \mathrm{a}=10.4940(5) \AA & \alpha=90^{\circ} \\ & \mathrm{b}=12.6978(6) \AA & \beta=91.044(2)^{\circ} \\ & \mathrm{c}=15.9846(7) \AA & \gamma=90^{\circ} \\ \text { Volume } & 2129.61(17) \AA^{3} & \\ \text { Z } & 4 & \\ \text { Density (calculated) } & 1.368 \mathrm{~g} / \mathrm{cm}^{3} & \\ \text { Absorption coefficient } & 2.802 \mathrm{~mm}^{-1} & \\ \text { F(000) } & 912 & \end{array}$

Table S4. Data collection and structure refinement for compound 3.

\begin{tabular}{|c|c|}
\hline Diffractometer & Bruker D8 Venture Duo IMS \\
\hline Radiation source & IMS microsource, Mo \\
\hline $\begin{array}{l}\text { Theta range for data } \\
\text { collection }\end{array}$ & 2.30 to $29.57^{\circ}$ \\
\hline Index ranges & $-14<=\mathrm{h}<=14,-17<=\mathrm{k}<=17,-22<=1<=22$ \\
\hline Reflections collected & 66552 \\
\hline Independent reflections & $5978[\mathrm{R}(\mathrm{int})=0.0934]$ \\
\hline $\begin{array}{l}\text { Coverage of independent } \\
\text { reflections }\end{array}$ & $100.0 \%$ \\
\hline Absorption correction & Multi-Scan \\
\hline Max. and min. transmission & 0.7190 and 0.5600 \\
\hline Refinement method & Full-matrix least-squares on $\mathrm{F}^{2}$ \\
\hline Refinement program & SHELXL-2014/7 (Sheldrick, 2014) \\
\hline Function minimized & $\Sigma \mathrm{w}\left(\mathrm{F}_{\mathrm{o}}^{2}-\mathrm{F}_{\mathrm{c}}^{2}\right)^{2}$ \\
\hline Data / restraints / parameters & 5978 / 0 / 249 \\
\hline Goodness-of-fit on $F^{2}$ & 1.041 \\
\hline$\Delta / \sigma_{\max }$ & 0.002 \\
\hline \multirow[t]{2}{*}{ Final $\mathbf{R}$ indices } & $\begin{array}{l}\mathrm{R} 1=0.0348, w \mathrm{w} 2= \\
0.0645\end{array}$ \\
\hline & $\begin{array}{l}\mathrm{R} 1=0.0534, \mathrm{wR} 2= \\
0.0692\end{array}$ \\
\hline Weighting scheme & $\begin{array}{l}\mathrm{w}=1 /\left[\sigma^{2}\left(\mathrm{~F}_{\mathrm{o}}^{2}\right)+(0.0242 \mathrm{P})^{2}+1.9235 \mathrm{P}\right] \\
\text { where } \mathrm{P}=\left(\mathrm{F}_{\mathrm{o}}^{2}+2 \mathrm{~F}_{\mathrm{c}}^{2}\right) / 3\end{array}$ \\
\hline
\end{tabular}


Largest diff. peak and hole $\quad 0.461$ and $-0.386 \mathrm{e}^{-3}$

R.M.S. deviation from mean $0.084 \mathrm{e}^{-3}$

\section{SC-XRD determination of compound 5 (CCDC 1936318)}

A clear yellow fragment-like specimen of $\mathrm{C}_{44} \mathrm{H}_{70} \mathrm{Al}_{2} \mathrm{~N}_{4} \mathrm{Si}_{2}$, approximate dimensions $0.136 \mathrm{~mm}$ x $0.137 \mathrm{~mm}$ x $0.203 \mathrm{~mm}$, was used for the X-ray crystallographic analysis. The X-ray intensity data were measured on a Bruker D8 Venture system equipped with a Helios optic monochromator and a Mo TXS rotating anode $(\lambda=0.71073 \AA$ ).

A total of 1443 frames were collected. The total exposure time was 3.56 hours. The frames were integrated with the Bruker SAINT software package using a narrow-frame algorithm. The integration of the data using a triclinic unit cell yielded a total of 42515 reflections to a maximum $\theta$ angle of $25.03^{\circ}$ ( $0.84 \AA$ resolution), of which 8029 were independent (average redundancy 5.295 , completeness $\left.=99.8 \%, \mathrm{R}_{\text {int }}=3.46 \%, \mathrm{R}_{\text {sig }}=2.41 \%\right)$ and $6684(83.25 \%)$ were greater than $2 \sigma\left(\mathrm{F}^{2}\right)$. The final cell constants of $\underline{\mathrm{a}}=11.8436(4) \AA, \underline{\mathrm{b}}=12.0036(5) \AA$, $\underline{\mathrm{c}}=$ 16.2833(6) $\AA, \alpha=87.653(2)^{\circ}, \beta=80.177(2)^{\circ}, \gamma=88.234(2)^{\circ}$, volume = 2278.41(15) $\AA^{3}$, are based upon the refinement of the XYZ-centroids of 9290 reflections above $20 \sigma(\mathrm{I})$ with $4.930^{\circ}$ $<2 \theta<51.39^{\circ}$. Data were corrected for absorption effects using the Multi-Scan method (SADABS). The ratio of minimum to maximum apparent transmission was 0.915 . The calculated minimum and maximum transmission coefficients (based on crystal size) are 0.9700 and

0.9800 .

The final anisotropic full-matrix least-squares refinement on $\mathrm{F}^{2}$ with 698 variables converged at $\mathrm{R} 1=4.64 \%$, for the observed data and $\mathrm{wR} 2=11.77 \%$ for all data. The goodness-of-fit was 1.040. The largest peak in the final difference electron density synthesis was $0.346 \mathrm{e}^{-} / \AA^{3}$ and the largest hole was $-0.339 \mathrm{e}^{-} / \AA^{3}$ with an RMS deviation of $0.048 \mathrm{e}^{-} / \AA^{3}$. On the basis of the final model, the calculated density was $1.115 \mathrm{~g} / \mathrm{cm}^{3}$ and $\mathrm{F}(000), 832 \mathrm{e}^{-}$.

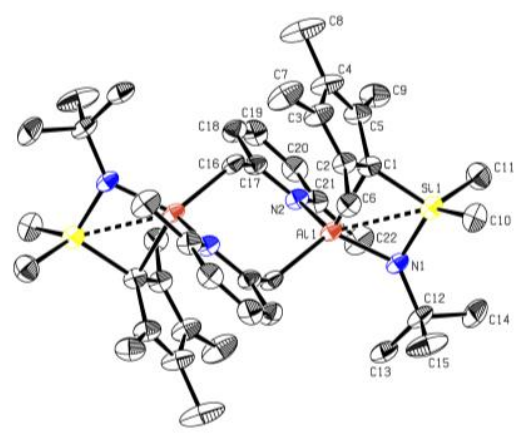

Figure S4. Ortep drawing with 50\% ellipsoids for compound 5. 
Table S5. Sample and crystal data for compound 5.

$\begin{array}{lll}\text { Identification code } & \text { WegMi6 } \mathrm{AP} 9203-100 & \\ \text { Chemical formula } & \mathrm{C}_{44} \mathrm{H}_{70} \mathrm{Al}_{2} \mathrm{~N}_{4} \mathrm{Si}_{2} & \\ \text { Formula weight } & 255.06 & \\ \text { Temperature } & 100(2) \mathrm{K} & \\ \text { Wavelength } & 0.71073 \AA & \\ \text { Crystal size } & 0.136 \times 0.137 \times 0.203 \mathrm{~mm} \\ \text { Crystal habit } & \text { clear yellow fragment } & \\ \text { Crystal system } & \text { triclinic } & \\ \text { Space group } & \mathrm{P}-1 & \alpha=87.653(2)^{\circ} \\ \text { Unit cell dimensions } & \mathrm{a}=11.8436(4) \AA & \beta=80.177(2)^{\circ} \\ & \mathrm{b}=12.0036(5) \AA & \\ & \mathrm{c}=16.2833(6) \AA & \gamma=88.234(2)^{\circ} \\ \text { Volume } & 2278.41(15) \AA^{3} & \\ \text { Z } & 6 & \\ \text { Density (calculated) } & 1.115 \mathrm{~g} / \mathrm{cm}^{3} & \\ \text { Absorption coefficient } & 0.150 \mathrm{~mm}^{-1} & \\ \text { F(000) } & 832 & \end{array}$

Table S6. Data collection and structure refinement for compound 5.

\begin{tabular}{|c|c|}
\hline Diffractometer & Bruker D8 Venture \\
\hline Radiation source & TXS rotating anode, Mo \\
\hline $\begin{array}{l}\text { Theta range for data } \\
\text { collection }\end{array}$ & 2.41 to $25.03^{\circ}$ \\
\hline Index ranges & $-14<=\mathrm{h}<=14,-14<=\mathrm{k}<=14,-19<=\mathrm{l}<=19$ \\
\hline Reflections collected & 42515 \\
\hline Independent reflections & $8029[\mathrm{R}(\mathrm{int})=0.0346]$ \\
\hline $\begin{array}{l}\text { Coverage of independent } \\
\text { reflections }\end{array}$ & $99.8 \%$ \\
\hline Absorption correction & Multi-Scan \\
\hline Max. and min. transmission & 0.9800 and 0.9700 \\
\hline Refinement method & Full-matrix least-squares on $\mathrm{F}^{2}$ \\
\hline Refinement program & SHELXL-2014/7 (Sheldrick, 2014) \\
\hline Function minimized & $\Sigma \mathrm{w}\left(\mathrm{F}_{\mathrm{o}}^{2}-\mathrm{F}_{\mathrm{c}}^{2}\right)^{2}$ \\
\hline Data / restraints / parameters & 8029 / 262 / 698 \\
\hline Goodness-of-fit on $\mathbf{F}^{2}$ & 1.040 \\
\hline$\Delta / \sigma_{\max }$ & 0.001 \\
\hline \multirow[t]{2}{*}{ Final $\mathbf{R}$ indices } & $\begin{array}{l}\mathrm{R} 1=0.0464, \mathrm{wR} 2= \\
0.1086\end{array}$ \\
\hline & $\begin{array}{l}\mathrm{R} 1=0.0592, \mathrm{wR} 2= \\
0.1177\end{array}$ \\
\hline Weighting scheme & $\begin{array}{l}\mathrm{w}=1 /\left[\sigma^{2}\left(\mathrm{~F}_{\mathrm{o}}^{2}\right)+(0.0399 \mathrm{P})^{2}+2.6556 \mathrm{P}\right] \\
\text { where } \mathrm{P}=\left(\mathrm{F}_{\mathrm{o}}^{2}+2 \mathrm{~F}_{\mathrm{c}}^{2}\right) / 3\end{array}$ \\
\hline
\end{tabular}


Largest diff. peak and hole $\quad 0.346$ and $-0.339 \mathrm{e}^{-3}$

R.M.S. deviation from mean $0.048 \mathrm{e}^{-3}$

\section{SC-XRD determination of compound 7 (CCDC 1936315)}

A clear colourless fragment-like specimen of $\mathrm{C}_{22} \mathrm{H}_{29} \mathrm{AlN}_{2} \mathrm{Si}$, approximate dimensions 0.126 $\mathrm{mm} \times 0.183 \mathrm{~mm} \times 0.389 \mathrm{~mm}$, was used for the X-ray crystallographic analysis. The X-ray intensity data were measured on a Bruker D8 Kappa Apex II system equipped with a Triumph monochromator monochromator and a Mo fine-focus sealed tube $(\lambda=0.71073 \AA$ ).

A total of 2682 frames were collected. The total exposure time was 3.73 hours. The frames were integrated with the Bruker SAINT software package using a narrow-frame algorithm. The integration of the data using a triclinic unit cell yielded a total of 35367 reflections to a maximum $\theta$ angle of $25.03^{\circ}(0.84 \AA$ resolution), of which 4254 were independent (average redundancy 8.314 , completeness $\left.=99.8 \%, \mathrm{R}_{\text {int }}=3.56 \%, \mathrm{R}_{\mathrm{sig}}=2.35 \%\right)$ and $3524(82.84 \%)$ were greater than $2 \sigma\left(\mathrm{F}^{2}\right)$. The final cell constants of $\underline{a}=9.558(2) \AA ⿻ 上 丨=9.687(2) \AA, \underline{c}=13.866(3) \AA$, $\alpha=106.254(9)^{\circ}, \beta=94.156(9)^{\circ}, \gamma=100.572(9)^{\circ}$, volume $=1201.1(5) \AA^{3}$, are based upon the refinement of the XYZ-centroids of 116 reflections above $20 \sigma(\mathrm{I})$ with $4.362^{\circ}<2 \theta<47.95^{\circ}$. Data were corrected for absorption effects using the Multi-Scan method (SADABS). The ratio of minimum to maximum apparent transmission was 0.950 . The calculated minimum and maximum transmission coefficients (based on crystal size) are 0.9470 and 0.9820 .

The final anisotropic full-matrix least-squares refinement on $\mathrm{F}^{2}$ with 241 variables converged at $\mathrm{R} 1=3.46 \%$, for the observed data and $\mathrm{wR} 2=8.68 \%$ for all data. The goodness-of-fit was 1.036. The largest peak in the final difference electron density synthesis was $0.272 \mathrm{e}^{-} / \AA^{3}$ and the largest hole was $-0.232 \mathrm{e}^{-} / \AA^{3}$ with an RMS deviation of $0.040 \mathrm{e}^{-1} / \AA^{3}$. On the basis of the final model, the calculated density was $1.041 \mathrm{~g} / \mathrm{cm}^{3}$ and $\mathrm{F}(000), 404 \mathrm{e}$.

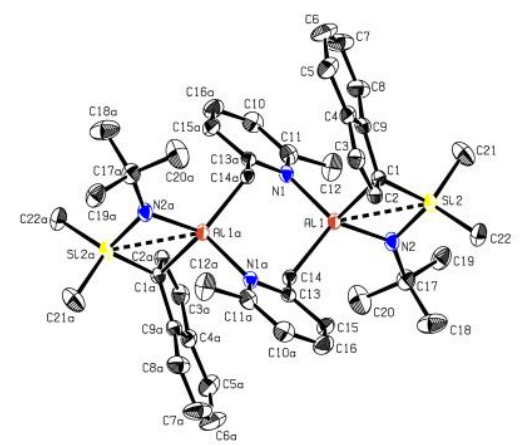

Figure S5. Ortep drawing with 50\% ellipsoids for compound 7. 
Table S7. Sample and crystal data for compound 7.

$\begin{array}{lll}\text { Identification code } & \mathrm{SchFa} 2 \mathrm{AP} 7596-100 \\ \text { Chemical formula } & \mathrm{C}_{22} \mathrm{H}_{29} \mathrm{AlN}_{2} \mathrm{Si} & \\ \text { Formula weight } & 376.54 \\ \text { Temperature } & 100(2) \mathrm{K} \\ \text { Wavelength } & 0.71073 \AA \\ \text { Crystal size } & 0.126 \times 0.183 \times 0.389 \mathrm{~mm} \\ \text { Crystal habit } & \text { clear colourless fragment } \\ \text { Crystal system } & \text { triclinic } & \\ \text { Space group } & \mathrm{P}-1 & \\ \text { Unit cell dimensions } & \mathrm{a}=9.558(2) \AA & \alpha=106.254(9)^{\circ} \\ & \mathrm{b}=9.687(2) \AA & \beta=94.156(9)^{\circ} \\ & \mathrm{c}=13.866(3) \AA & \gamma=100.572(9)^{\circ} \\ \text { Volume } & 1201.1(5) \AA^{3} & \\ \text { Z } & 2 & \\ \text { Density (calculated) } & 1.041 \mathrm{~g} / \mathrm{cm}^{3} & \\ \text { Absorption coefficient } & 0.141 \mathrm{~mm}^{-1} & \\ \text { F(000) } & 404\end{array}$

Table S8. Data collection and structure refinement for compound 7.

\begin{tabular}{|c|c|c|}
\hline & \multirow{2}{*}{\multicolumn{2}{|c|}{$\begin{array}{l}\text { Bruker D8 Kappa Apex II } \\
\text { fine-focus sealed tube, Mo }\end{array}$}} \\
\hline Radiation source & & \\
\hline $\begin{array}{l}\text { Theta range for data } \\
\text { collection }\end{array}$ & \multicolumn{2}{|c|}{2.19 to $25.03^{\circ}$} \\
\hline Index ranges & \multicolumn{2}{|c|}{$-11<=\mathrm{h}<=11,-11<=\mathrm{k}<=11,-16<=1<=16$} \\
\hline Reflections collected & \multicolumn{2}{|l|}{35367} \\
\hline Independent reflections & \multicolumn{2}{|c|}{$4254[\mathrm{R}(\mathrm{int})=0.0356]$} \\
\hline $\begin{array}{l}\text { Coverage of independent } \\
\text { reflections }\end{array}$ & \multicolumn{2}{|l|}{$99.8 \%$} \\
\hline Absorption correction & \multicolumn{2}{|l|}{ Multi-Scan } \\
\hline Max. and min. transmission & \multicolumn{2}{|c|}{0.9820 and 0.9470} \\
\hline Refinement method & \multicolumn{2}{|c|}{ Full-matrix least-squares on $\mathrm{F}^{2}$} \\
\hline Refinement program & \multicolumn{2}{|c|}{ SHELXL-2014/7 (Sheldrick, 2014) } \\
\hline Function minimized & \multicolumn{2}{|c|}{$\Sigma \mathrm{w}\left(\mathrm{F}_{\mathrm{o}}^{2}-\mathrm{F}_{\mathrm{c}}^{2}\right)^{2}$} \\
\hline Data / restraints / parameters & \multicolumn{2}{|c|}{4254 / 0 / 241} \\
\hline Goodness-of-fit on $\mathbf{F}^{2}$ & \multicolumn{2}{|l|}{1.036} \\
\hline \multirow[t]{2}{*}{ Final $\mathbf{R}$ indices } & $\begin{array}{l}3524 \text { data; } \\
\mathrm{I}>2 \sigma(\mathrm{I})\end{array}$ & $\begin{array}{l}\mathrm{R} 1=0.0346, \mathrm{wR} 2= \\
0.0811\end{array}$ \\
\hline & all data & $\begin{array}{l}\mathrm{R} 1=0.0447, \mathrm{wR} 2= \\
0.0868\end{array}$ \\
\hline Weighting scheme & \multicolumn{2}{|c|}{$\begin{array}{l}\mathrm{w}=1 /\left[\sigma^{2}\left(\mathrm{~F}_{\mathrm{o}}^{2}\right)+(0.0349 \mathrm{P})^{2}+0.6634 \mathrm{P}\right] \\
\text { where } \mathrm{P}=\left(\mathrm{F}_{\mathrm{o}}^{2}+2 \mathrm{~F}_{\mathrm{c}}^{2}\right) / 3\end{array}$} \\
\hline Largest diff. peak and hole & \multicolumn{2}{|c|}{0.272 and $-0.232 \mathrm{e}^{-3}$} \\
\hline
\end{tabular}


R.M.S. deviation from mean $0.040 \mathrm{e}^{-3}$ 


\section{Polymerization Studies and Mechanism Elucidation}

Further polymerization experiments in addition to Table 1:

Table S9. Catalytic conversion of DEVP with the synthesized CGCs. ${ }^{\text {(a) }}$

\begin{tabular}{lcccccccc}
\hline Run & Catalyst & $\mathrm{V}_{\text {tol }} / \mathrm{mL}$ & $\mathrm{t} / \mathrm{min}$ & ${\text { Yield } / \%{ }^{(\mathrm{b})}}$ & $\mathrm{M}_{\mathrm{n}} / 10^{3} \mathrm{~g} / \mathrm{mol}^{(\mathrm{c})}$ & $\mathrm{D}\left(\mathrm{M}_{\mathrm{w}} / \mathrm{M}_{\mathrm{n}}\right)$ & $\mathrm{I} / \%^{(\mathrm{d})}$ & $\mathrm{TOF} / \mathrm{h}^{-1}$ \\
\hline 1 & $\mathbf{1 - C H _ { 2 } \text { TMS }}$ & 20 & 1 & 100 & 150 & 1.19 & 32 & 18,000 \\
2 & 2-CH2TMS & 20 & 1 & 100 & 450 & 1.26 & 11 & 18,000 \\
3 & $\mathbf{5}$ & 3 & 45 & 91 & 160 & 1.83 & 28 & 360 \\
4 & $\mathbf{6}$ & 3 & 45 & 38 & 160 & 1.67 & 10 & 150 \\
5 & 7 & 3 & 45 & 98 & 100 & 2.28 & 48 & 400 \\
\hline
\end{tabular}

(a) $\mathrm{V}_{\mathrm{DEVP}}=1.0 \mathrm{~mL}, \mathrm{~T}=30{ }^{\circ} \mathrm{C},[\mathrm{M}] /[\mathrm{CGC}]=300 / 1$, (b) measured gravimetrically and by NMR spectroscopy $\left({ }^{31} \mathrm{P}\right.$ NMR spectroscopy), (c) determined by GPC-MALS in $\mathrm{H}_{2} \mathrm{O} / \mathrm{THF}(9 \mathrm{~g} / \mathrm{L}$ tetrabutylammonium bromide), (d) initiator efficiency $\left(\mathrm{M}_{\mathrm{n}(\text { theo. })} / \mathrm{M}_{\mathrm{n}(\mathrm{determ} .)}\right)$.

End group analysis via ESI-MS:

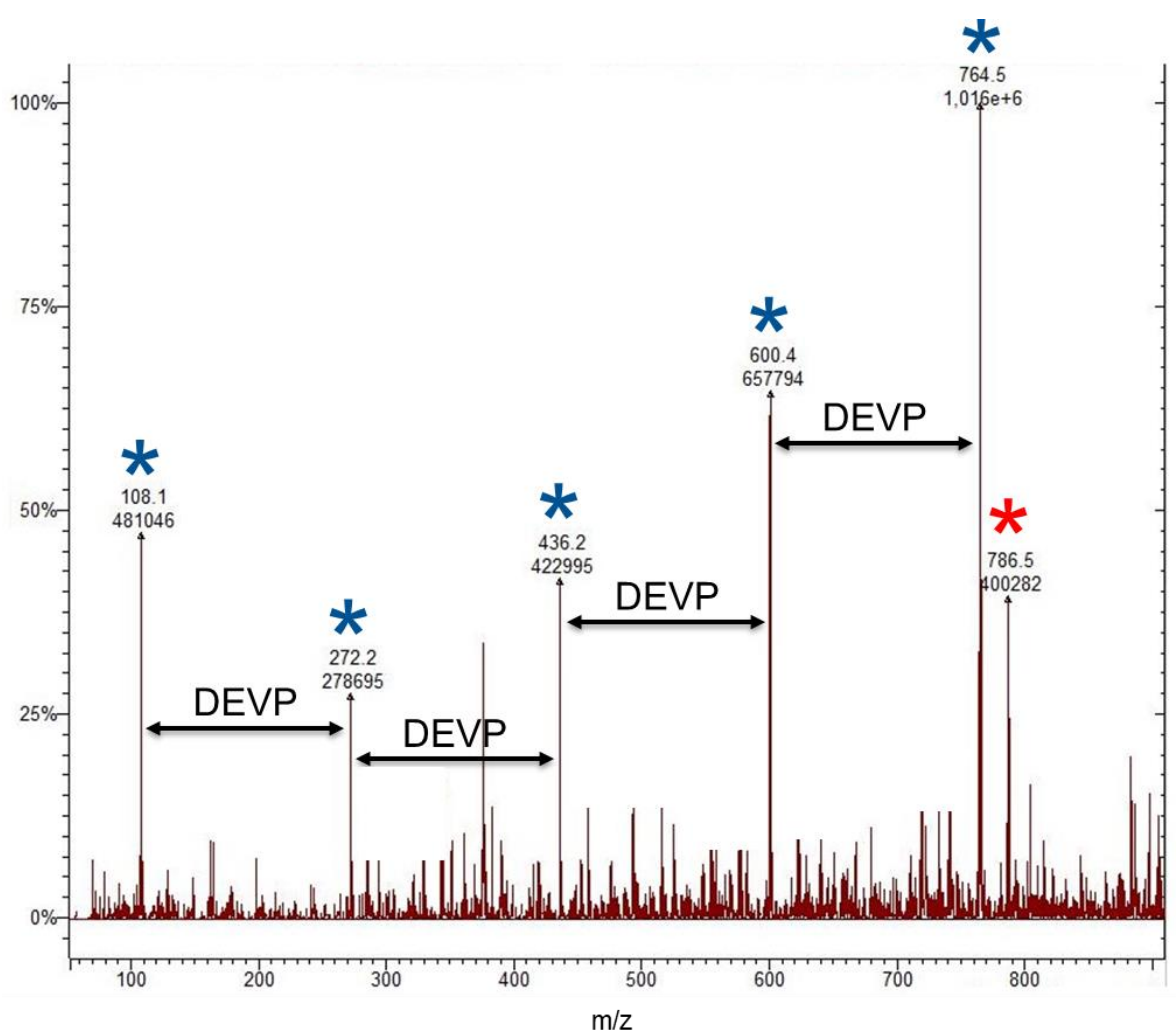

Figure S6. ESI MS spectrum of DEVP oligomers produced with 5. One major series of peaks is evident: $\mathrm{m} / \mathrm{z}=\mathrm{n} \times \mathrm{M}_{\mathrm{Mon}}+\mathrm{M}_{\mathrm{H}}+\mathrm{M}_{\text {Lut }}$ (blue); $\mathrm{M}_{\mathrm{Mon}}=164$, end groups: $M_{\text {Lut }}=107$. Red peak: $\mathrm{m} / \mathrm{z}=\mathrm{n} \times \mathrm{M}_{\mathrm{Mon}}$ $+\mathrm{M}_{\mathrm{Na}}+\mathrm{M}_{\mathrm{Lut}} ; \mathrm{M}_{\mathrm{Mon}}=164$, end groups: $M_{\mathrm{Lut}}=107$. 


\section{Living polymerization behaviour (CGC 5):}

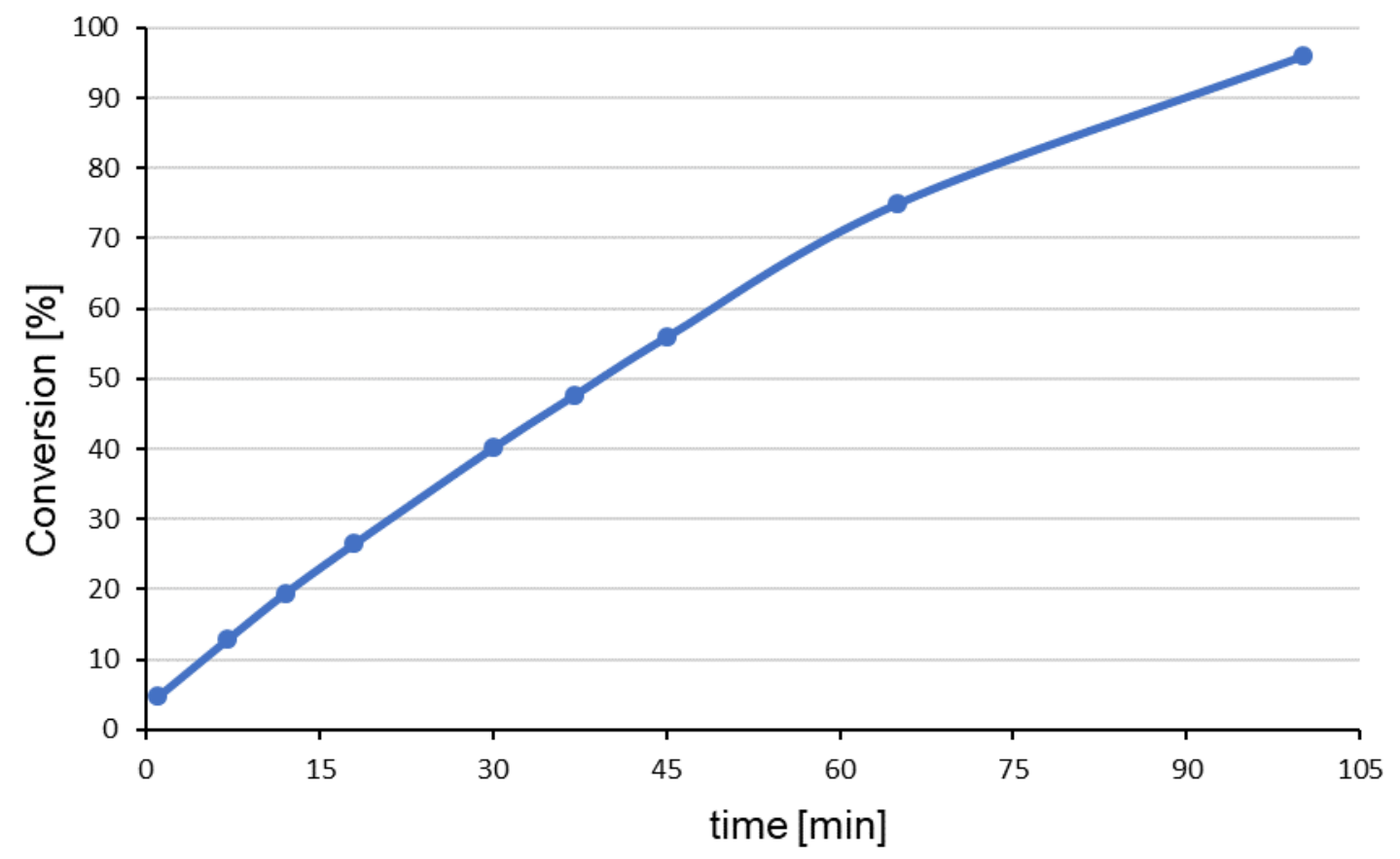

Figure S7. Time vs. conversion plot for polymerization of diethylvinylphosphonate with $\mathbf{5}$ (Monomer to catalyst ratio of $300 / 1$, r.t., $5 \mathrm{~mL}$ toluene, $0.5 \mathrm{~mL} \mathrm{DEVP}$ ).

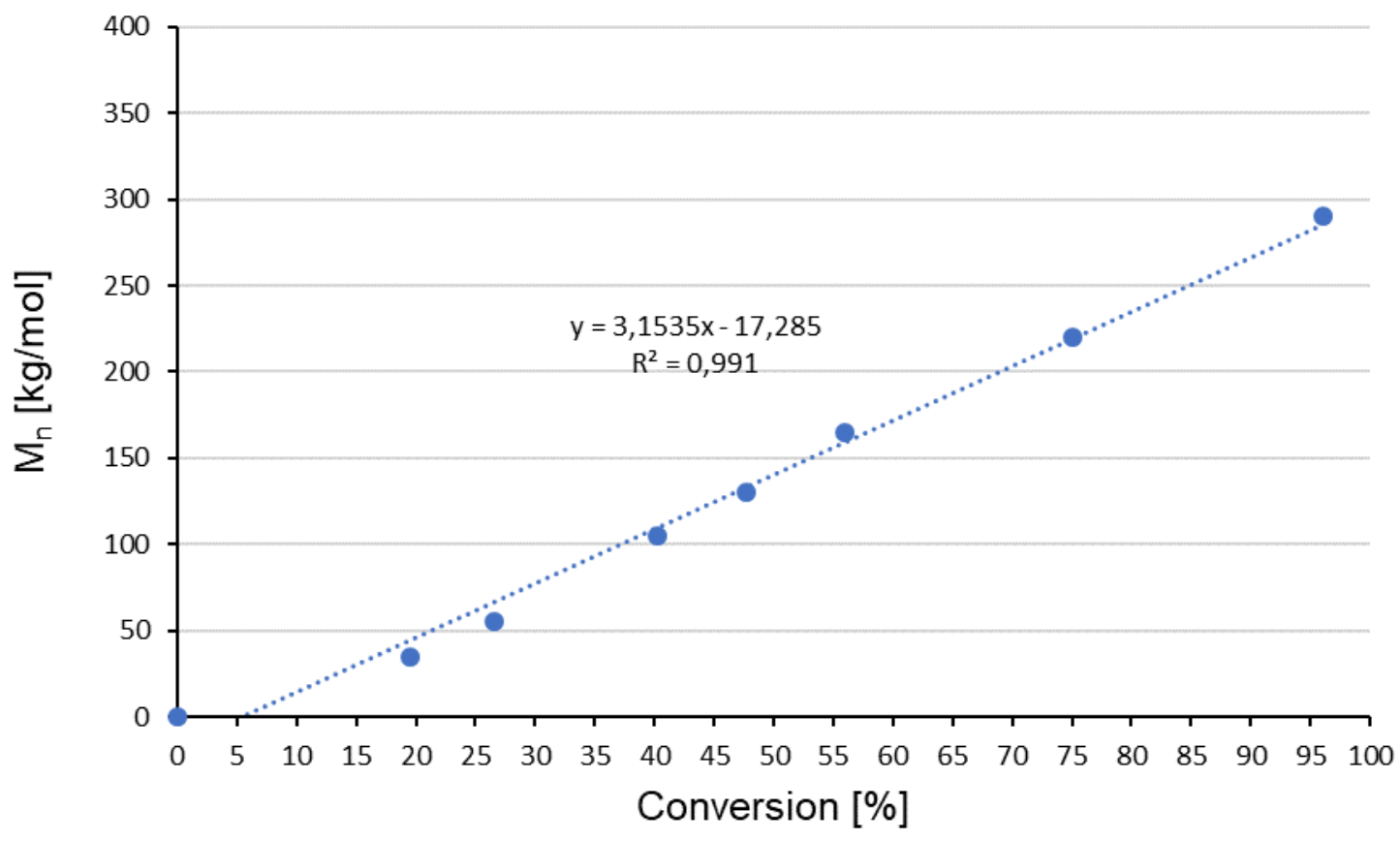

Figure S8. Linear growth of the molecular weight with increasing conversion. 
Determination of catalyst order (differential method):

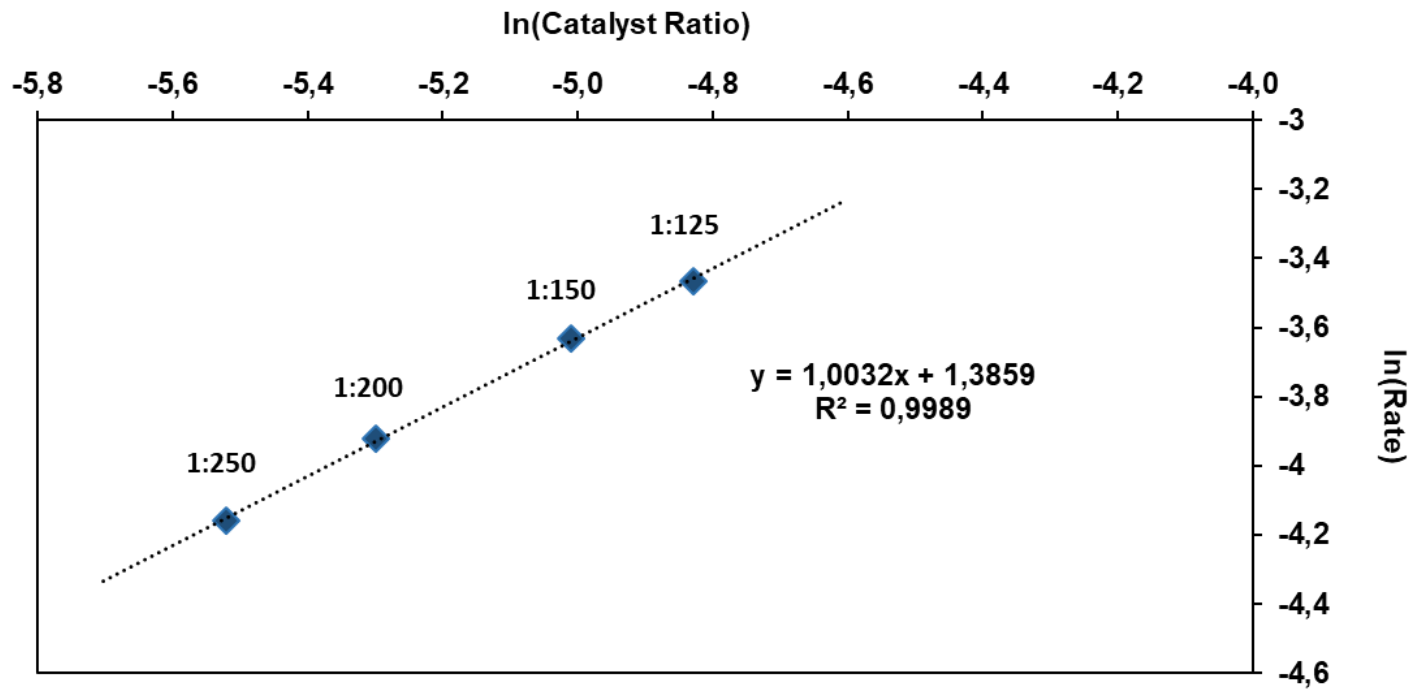

Figure S9. Determination of catalyst order: catalyst 5, $0.15 \mathrm{~mL}$ DEVP, $0.75 \mathrm{~mL} \mathrm{C}_{6} \mathrm{D}_{6}, 25{ }^{\circ} \mathrm{C}$.

Determination of monomer order (tangential method):

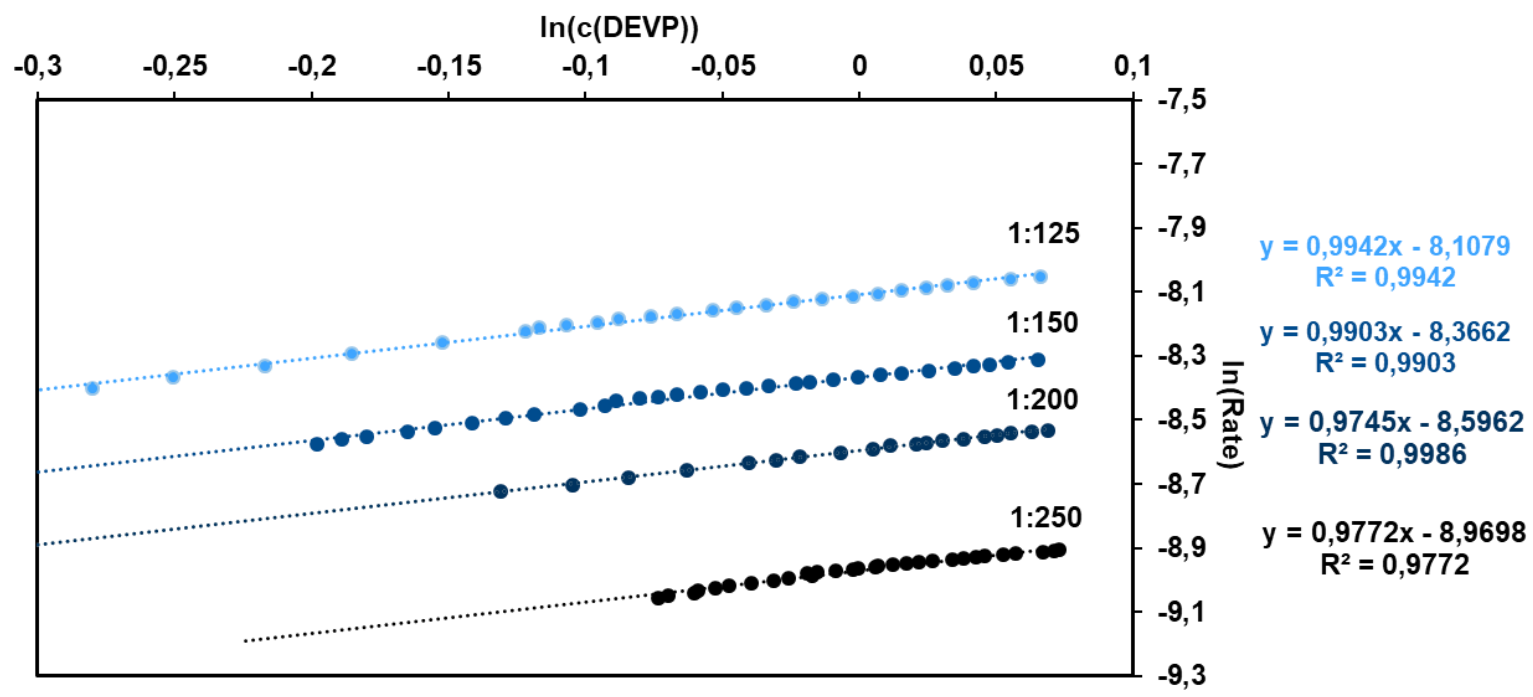

Figure S10. Determination of monomer order: catalyst 5, $0.15 \mathrm{~mL}$ DEVP, $0.75 \mathrm{~mL} \mathrm{C}_{6} \mathrm{D}_{6}, 25{ }^{\circ} \mathrm{C}$. 


\section{Living polymerization behaviour (CGC 1):}

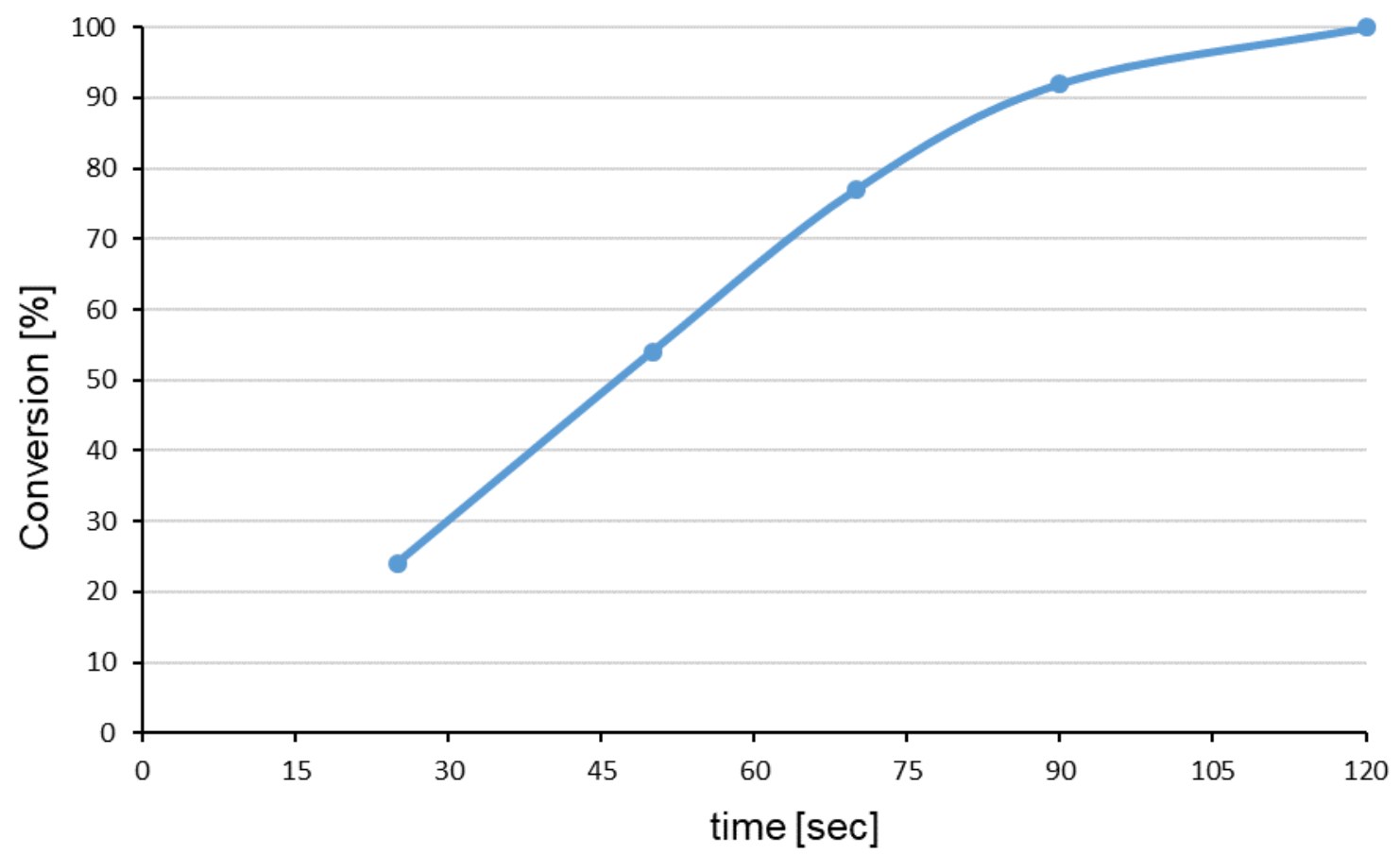

Figure S11. Time vs. conversion plot for polymerization of diethylvinylphosphonate with 1 (Monomer to catalyst ratio of $300 / 1,-30{ }^{\circ} \mathrm{C}, 20 \mathrm{~mL}$ toluene, $1 \mathrm{~mL}$ DEVP).

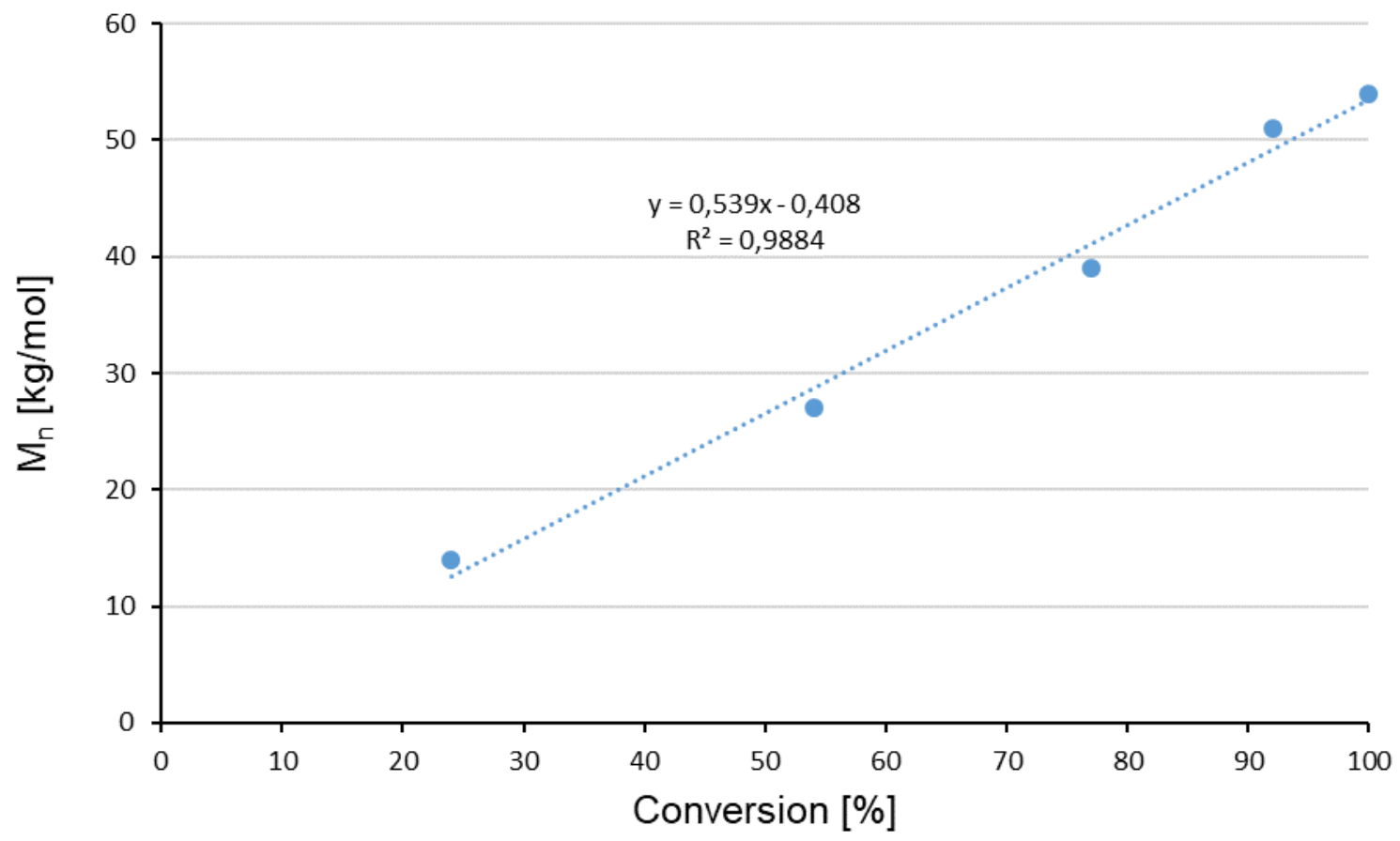

Figure S12. Linear growth of the molecular weight with increasing conversion. 


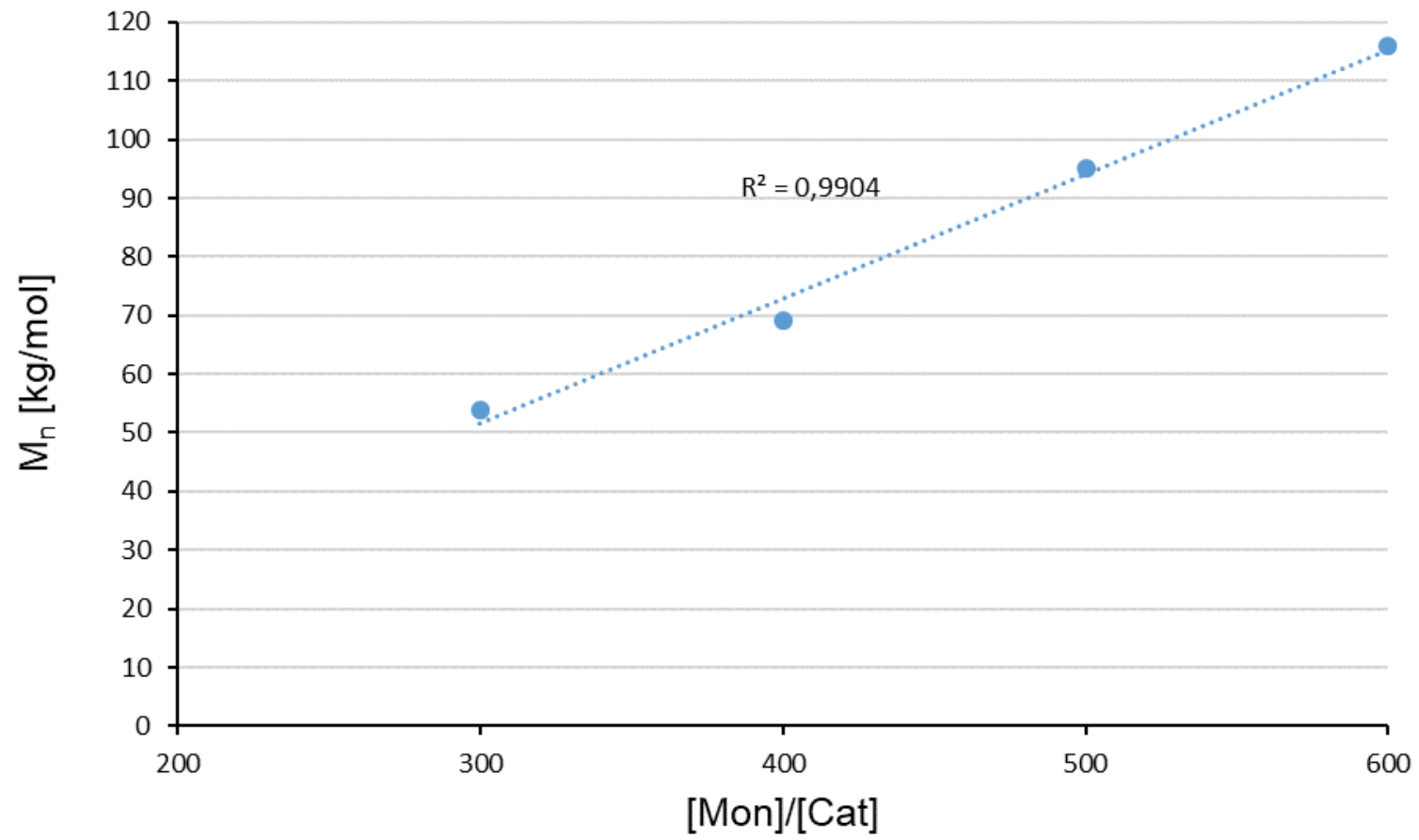

Figure S13. Molecular weight vs. [Mon]/[Cat] plot for polymerization of diethylvinylphosphonate with 1 ensuring quantitative conversions $\left(-30^{\circ} \mathrm{C}, 20 \mathrm{~mL}\right.$ toluene, $1 \mathrm{~mL}$ DEVP).

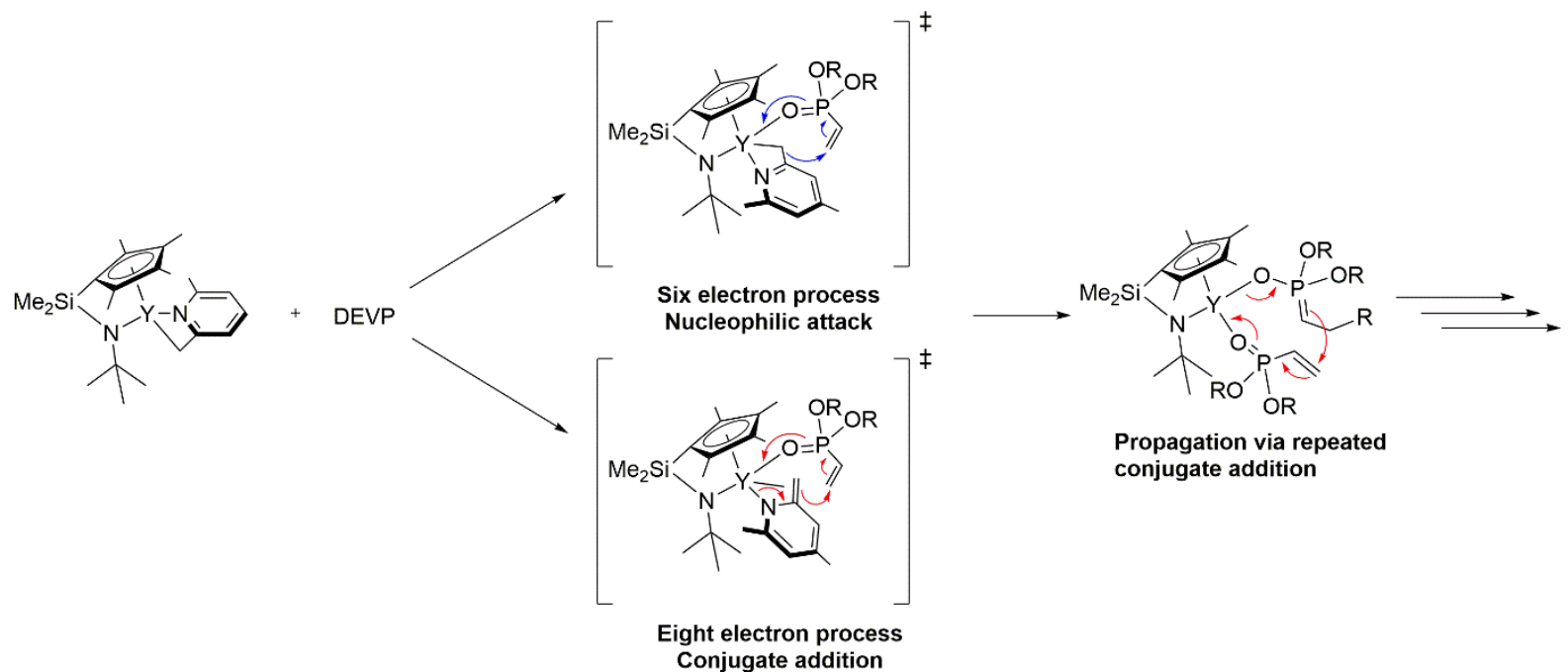

Scheme S1. Proposed initiation reaction via nucleophilic transfer (six electron process, blue) or conjugate addition (eight electron process, red) and propagation via a repeated conjugate addition of the polymer chain to the coordinated monomer at the metal center by the example of catalyst $\mathbf{1}$. 


\section{NMR, GPC and Thermal Analysis of the PDEVP Samples}

\section{Comparison of the ${ }^{31} \mathbf{P}\left\{{ }^{1} \mathbf{H}\right\}$ NMR spectra:}

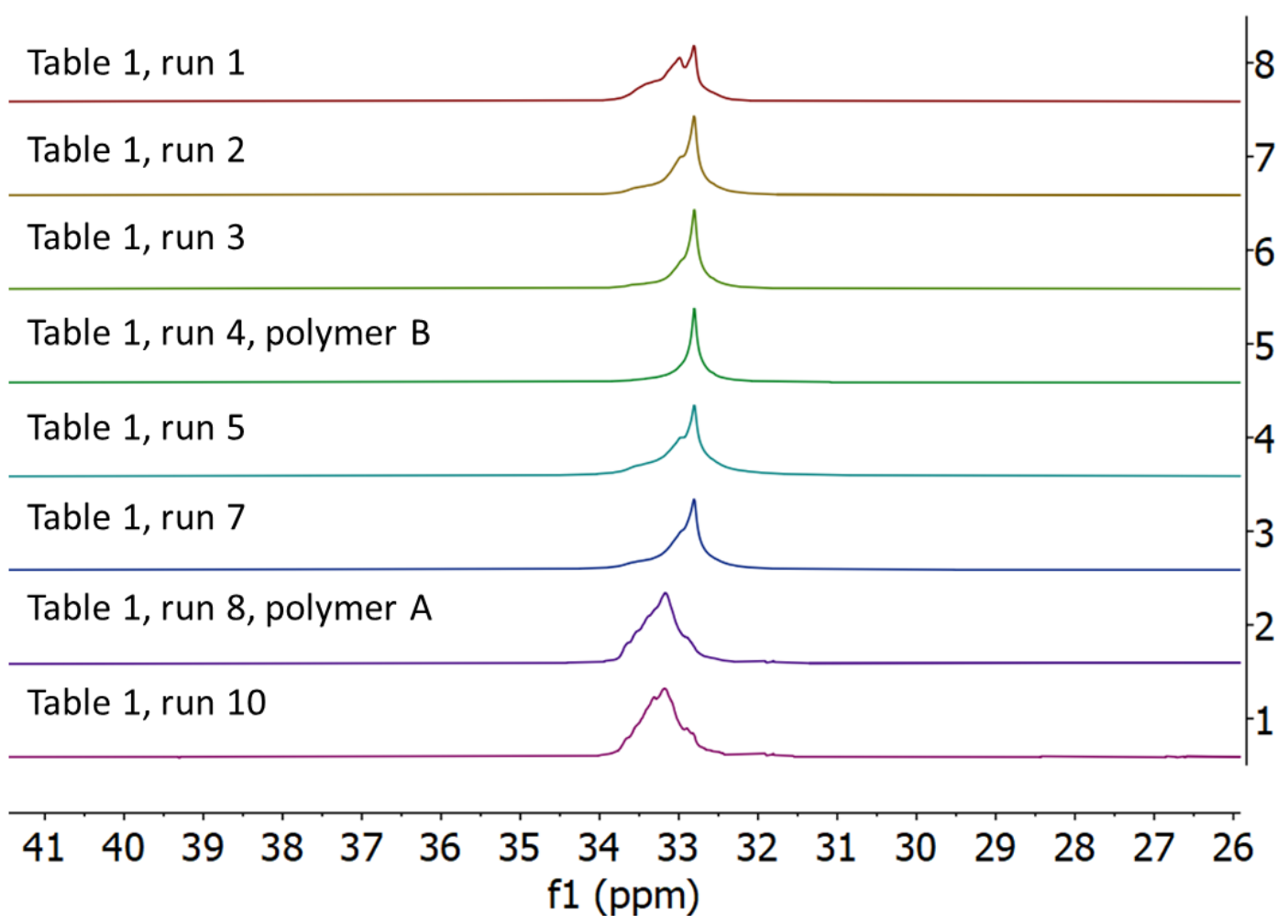

Figure S14. ${ }^{31} \mathrm{P}\left\{{ }^{1} \mathrm{H}\right\}$ NMR spectra of the discussed polymers in $\mathrm{MeOD}_{4}$.

NMR spectra, thermal characteristics \& GPC trace of Table 1, run 4 (polymer B):

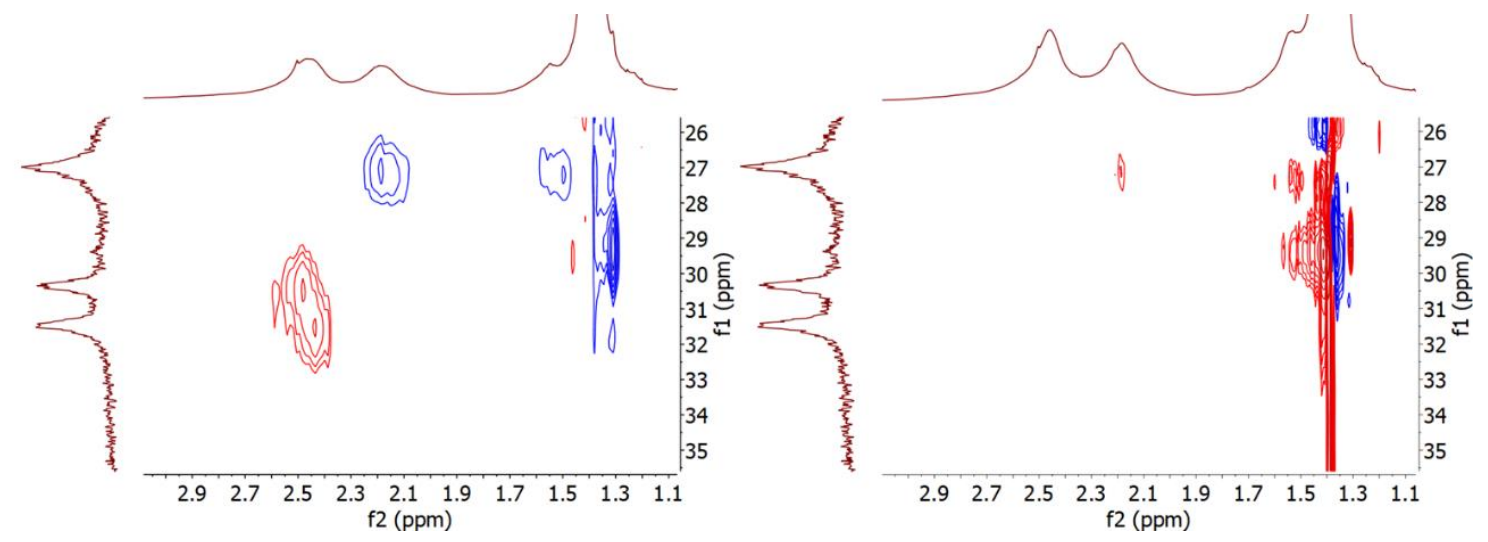

Figure S15. Phase-sensitive ${ }^{1} \mathrm{H}-{ }^{13} \mathrm{C}$ DEPT-edited HSQC vs. a ${ }^{1} \mathrm{H}_{-}{ }^{13} \mathrm{C}-{ }^{31} \mathrm{P}$ triple resonance $\mathrm{HCP} 2 \mathrm{D}$ experiment edited by $J_{\mathrm{PC}}=20 \mathrm{~Hz}$ (for details see Table S10). 
Table S10. Pulseprogram parameters for ${ }^{1} \mathrm{H}^{13} \mathrm{C}^{31} \mathrm{P}$ triple resonance $\mathrm{HCP} 2 \mathrm{D}$ experiment edited by $\mathbf{J}_{\mathrm{PC}}=20 \mathrm{~Hz}$ (Figure $\left.\mathbf{S} 12\right)$.

General

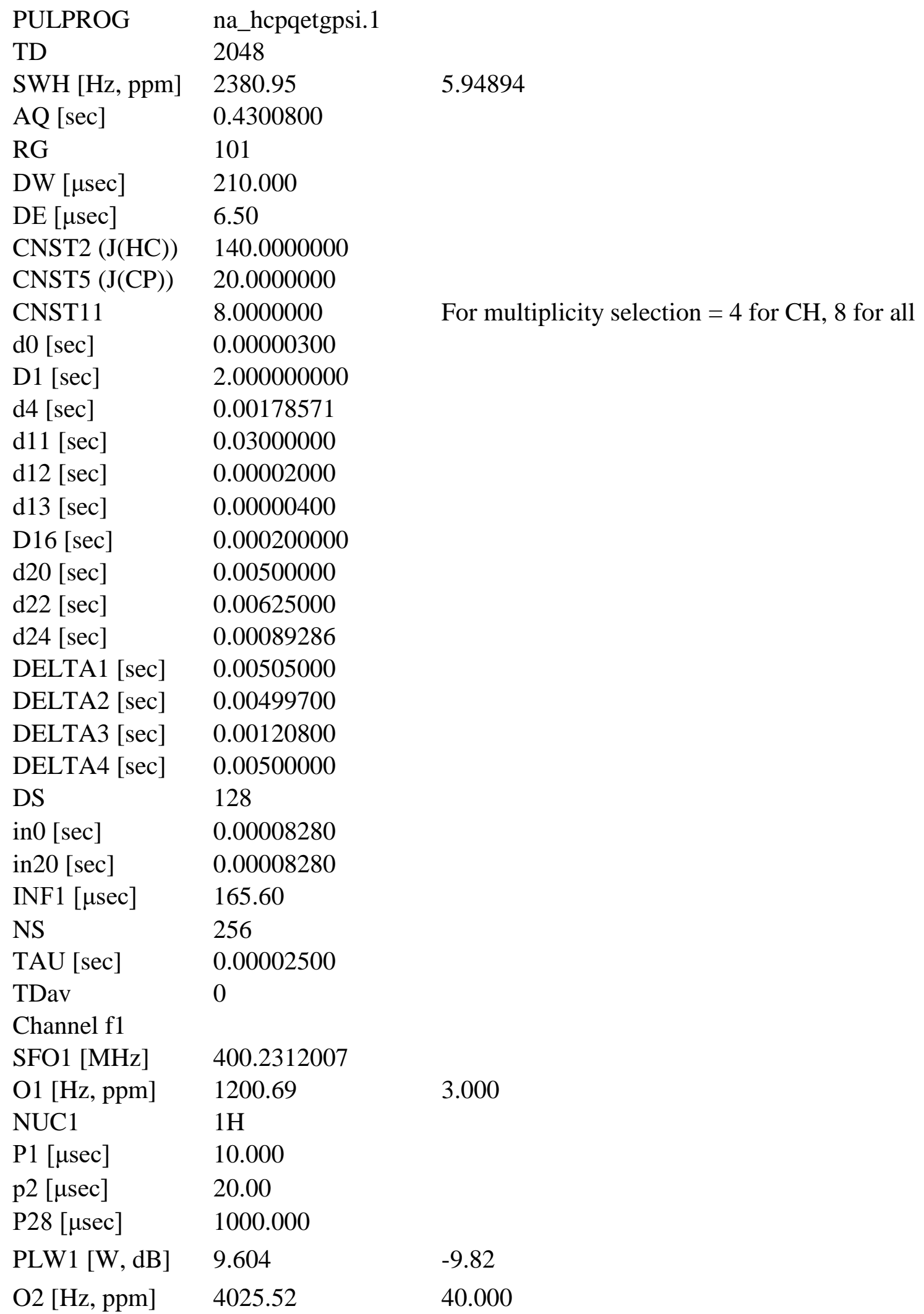




\begin{tabular}{|c|c|c|}
\hline NUC2 & $13 \mathrm{C}$ & \\
\hline CPDPRG 2 & garp4 & \\
\hline $\mathrm{P} 3[\mu \mathrm{sec}]$ & 20.000 & \\
\hline $\mathrm{p} 4[\mu \mathrm{sec}]$ & 40.00 & \\
\hline PCPD2 [ $\mu \mathrm{sec}]$ & 80.00 & \\
\hline PLW2 [W, dB] & 52.44 & -17.20 \\
\hline PLW12 [W, dB] & 3.2775 & -5.16 \\
\hline \multicolumn{3}{|l|}{ Channel f3 } \\
\hline SFO3 [MHz] & 162.0210965 & \\
\hline $\mathrm{O} 3$ [Hz, ppm] & 5022.50 & 31.000 \\
\hline NUC3 & $31 \mathrm{P}$ & \\
\hline CPDPRG 3 & garp4 & \\
\hline $\mathrm{P} 21[\mu \mathrm{sec}]$ & 25.000 & \\
\hline p22 [ $\mu$ sec $]$ & 50.00 & \\
\hline PCPD3 [ $\mu \mathrm{sec}]$ & 100.00 & \\
\hline PLW3 [W, dB] & 15.924 & -12.02 \\
\hline PLW16 [W, dB] & 0.99525 & 0.02 \\
\hline \multicolumn{3}{|l|}{ Gradient channel } \\
\hline GPNAM 1 & SMSQ10.100 & \\
\hline GPZ1 [\%] & 40.00 & \\
\hline GPNAM 2 & SMSQ10.100 & \\
\hline GPZ2 [\%] & 25.00 & \\
\hline GPNAM 3 & SMSQ10.100 & \\
\hline GPZ3 [\%] & 40.00 & \\
\hline GPNAM 4 & SMSQ10.100 & \\
\hline GPZ4 [\%] & 20.10 & \\
\hline $\mathrm{P} 16[\mu \mathrm{sec}]$ & 1000.000 & \\
\hline
\end{tabular}



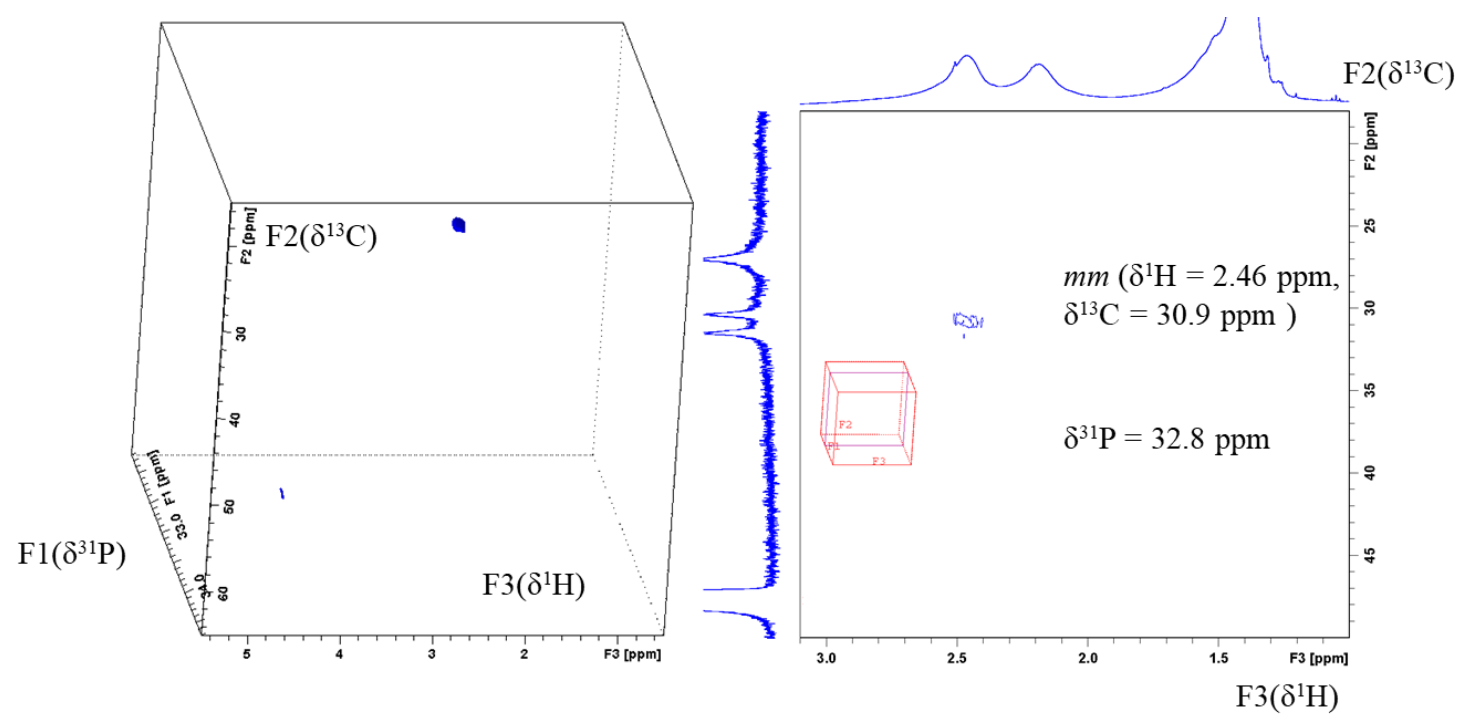

Figure S16. ${ }^{1} \mathrm{H}-{ }^{13} \mathrm{C}-{ }^{31} \mathrm{P}$ triple resonance $\mathrm{HCP}$ 3D experiment edited by $J_{\mathrm{PC}}=110 \mathrm{~Hz}$ (for details see Table S11, S12).

Table S11. Pulseprogram for ${ }^{1} \mathrm{H}_{-}{ }^{13} \mathrm{C}-{ }^{31} \mathrm{P}$ triple resonance HCP $3 \mathrm{D}$ experiment edited by $J_{\mathrm{PC}}=$ $110 \mathrm{~Hz}$ (Figure S13, SX).

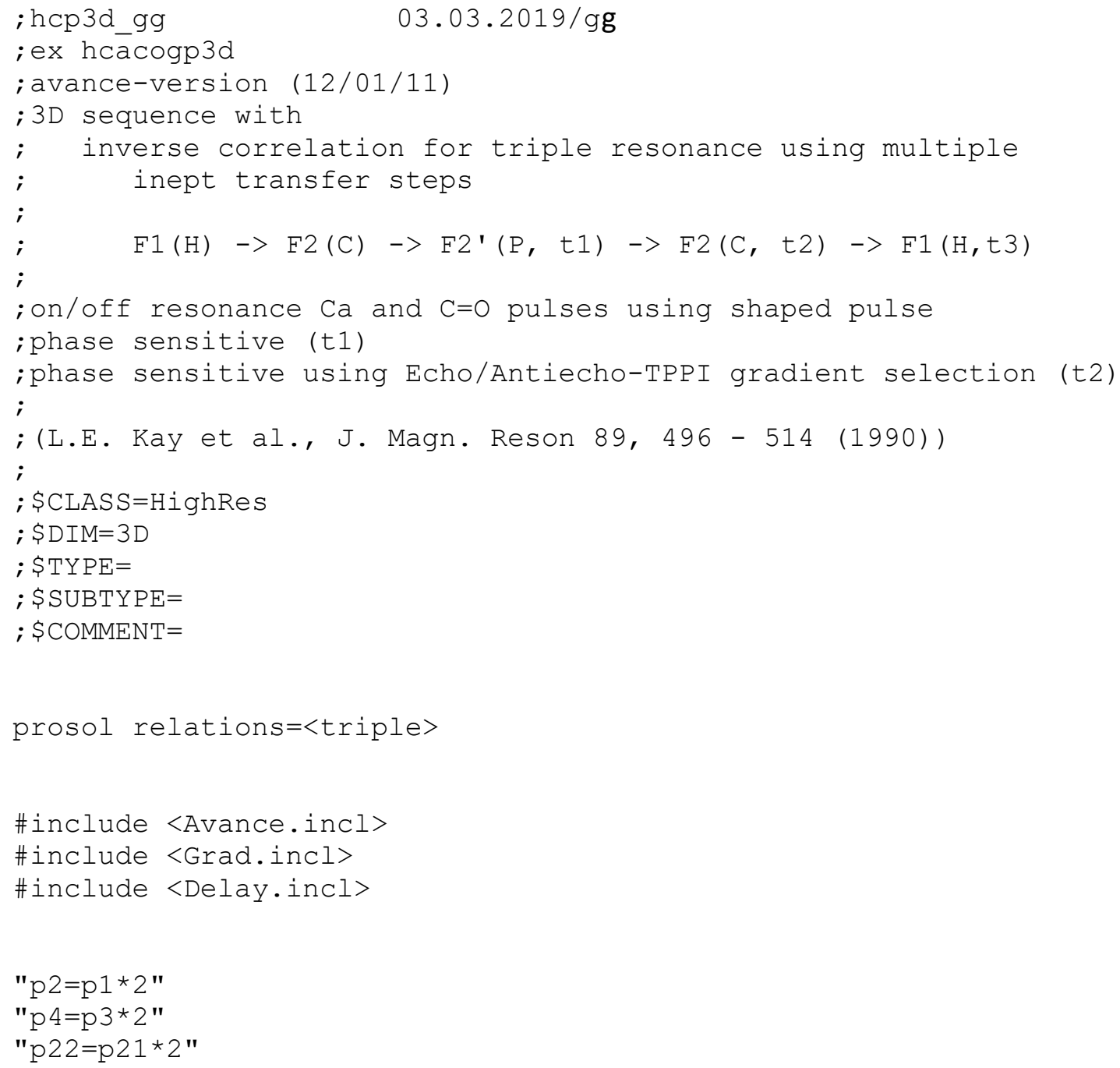




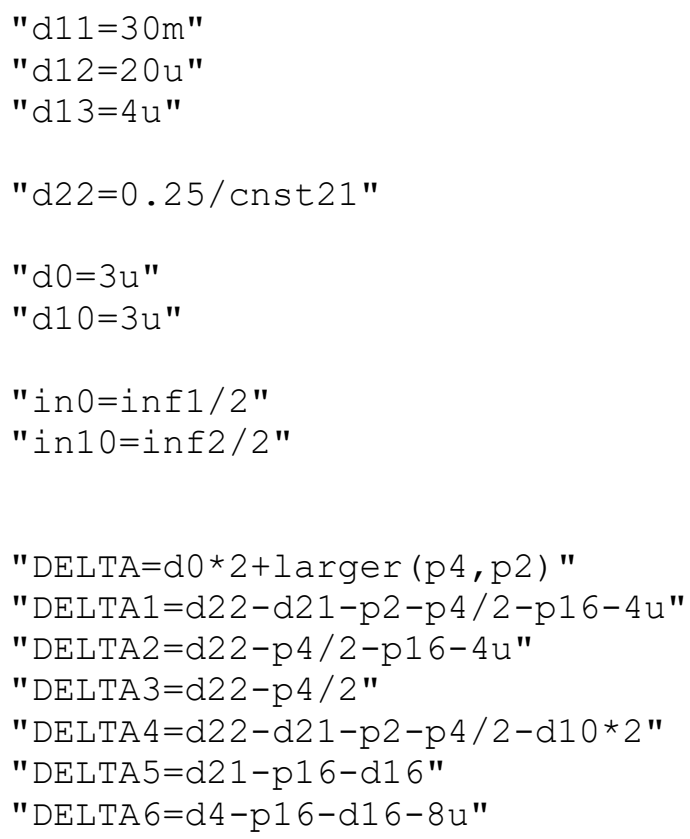




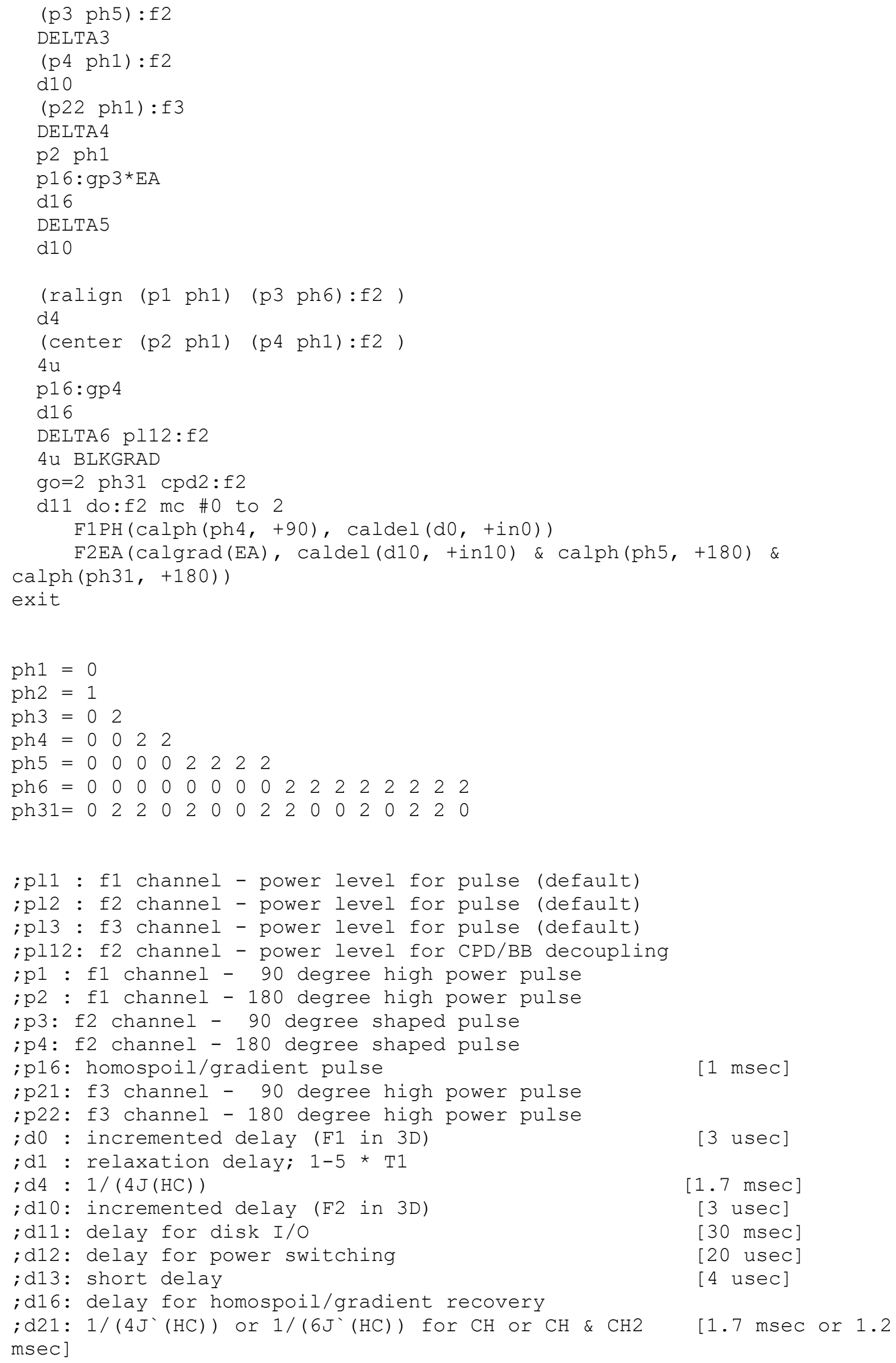




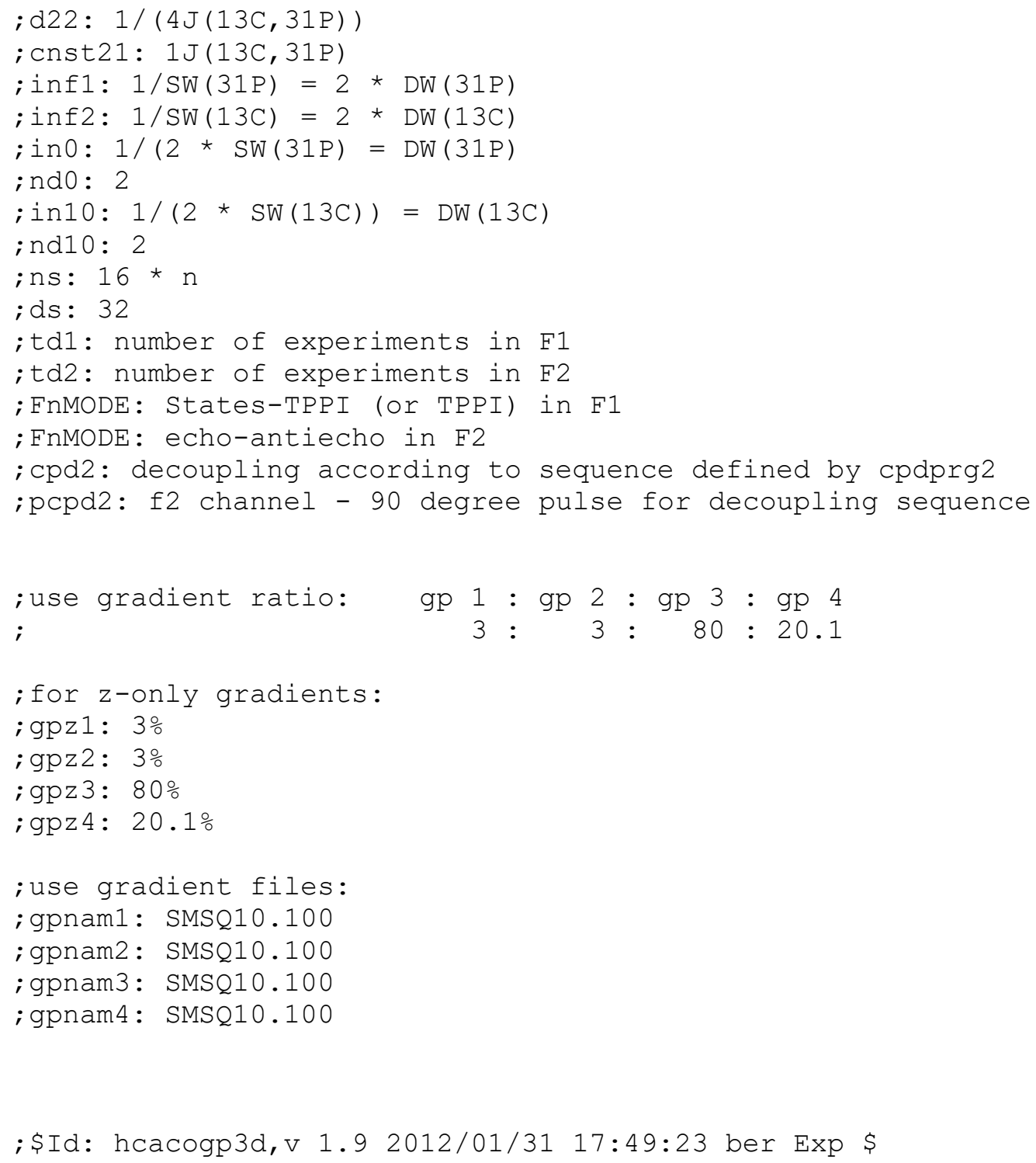

Table S12. Pulseprogram parameters for ${ }^{1} \mathrm{H}_{-}{ }^{13} \mathrm{C}-{ }^{31} \mathrm{P}$ triple resonance $\mathrm{HCP}$ 3D experiment edited by $J_{\mathrm{PC}}=110 \mathrm{~Hz}$ (Figure $\left.S 13, \mathrm{SX}\right)$.

\section{General}

PULPROG

TD

SWH $[\mathrm{Hz}, \mathrm{ppm}]$

$\mathrm{AQ}$ [sec]

RG

DW $[\mu \mathrm{sec}]$

$\mathrm{DE}[\mu \mathrm{sec}]$

CNST21 (1J13C,31P)

d0 [sec]

D1 [sec]

D4 [sec]

d10 [sec] hcp3d_gg

2048

2000.00

0.5120000

101

250.000

6.50

110.0000000

0.00000300

2.000000000

0.001700000

0.00000300 


$\begin{array}{ll}\mathrm{d} 11 \text { [sec] } & 0.03000000 \\ \mathrm{~d} 13 \text { [sec] } & 0.00000400 \\ \mathrm{D} 16 \text { [sec] } & 0.000200000 \\ \mathrm{D} 21 \text { [sec] } & 0.001200000 \\ \mathrm{~d} 22 \text { [sec] } & 0.00227273 \\ \text { DELTA [sec] } & 0.00004600 \\ \text { DELTA1 [sec] } & 0.00002873 \\ \text { DELTA2 [sec] } & 0.00124873 \\ \text { DELTA3 [sec] } & 0.00225273 \\ \text { DELTA4 [sec] } & 0.00102673 \\ \text { DELTA5 [sec] } & 0 \\ \text { DELTA6 [sec] } & 0.00049200 \\ \text { DS } & 32 \\ \text { in0 [sec] } & 0.00102870 \\ \text { in10 [sec] } & 0.00009940 \\ \text { INF1 [ } \mu \mathrm{sec}] & 2057.40 \\ \text { INF2 [ } \mu \mathrm{sec}] & 198.80 \\ \text { NS } & 48 \\ \text { TDav } & 0 \\ \text { Cha }\end{array}$

Channel f1

SFO1 [MHz]

O1 [Hz, ppm]

NUC1

$\mathrm{P} 1$ [ $\mu \mathrm{sec}]$

p2 [ $\mu \mathrm{sec}]$

PLW1 [W, dB]

Channel f2

$\mathrm{SFO} 2$ [MHz]

$\mathrm{O} 2[\mathrm{~Hz}, \mathrm{ppm}]$

NUC2

CPDPRG 2

$\mathrm{P} 3$ [ $\mu \mathrm{sec}]$

p4 [ $\mu \mathrm{sec}]$

PCPD2 [ $\mu \mathrm{sec}]$

PLW2 [W, dB]

PLW12 [W, dB]

Channel f3

SFO3 [MHz]

$\mathrm{O} 3[\mathrm{~Hz}, \mathrm{ppm}]$

NUC3

$\mathrm{P} 21[\mu \mathrm{sec}]$

p22 [ $\mu \mathrm{sec}]$

PLW3 [W, dB]

Gradient channel

GPNAM 1

GPZ1 [\%]

SMSQ10.100

3.00

$$
\begin{aligned}
& 0.03000000 \\
& 0.00000400 \\
& 0.000200000 \\
& 0.001200000 \\
& 0.00227273 \\
& 0.00004600 \\
& 0.00002873 \\
& 0.00124873 \\
& 0.00225273 \\
& 0.00102673 \\
& 0 \\
& 0.00049200 \\
& 32 \\
& 0.00102870 \\
& 0.00009940 \\
& 2057.40 \\
& 198.80 \\
& 48
\end{aligned}
$$

400.2312007

$1200.69 \quad 3.000$

$1 \mathrm{H}$

10.000

20.00

$9.604-9.82$

100.6419395

4025.52

40.000

$13 \mathrm{C}$

garp4

20.000

40.00

80.00

52.44

3.2775

$-17.20$

$-5.16$

162.0214529

5378.93

33.200

$31 \mathrm{P}$

25.000

50.00

15.924

$-12.02$ 


$\begin{array}{ll}\text { GPNAM 2 } & \text { SMSQ10.100 } \\ \text { GPZ2 [\%] } & 3.00 \\ \text { GPNAM 3 } & \text { SMSQ10.100 } \\ \text { GPZ3 [\%] } & 80.00 \\ \text { GPNAM 4 } & \text { SMSQ10.100 } \\ \text { GPZ4 [\%] } & 20.10 \\ \text { P16 }[\mu \mathrm{sec}] & 1000.000\end{array}$

\begin{tabular}{l|l} 
Peak & Area \\
\hline $2.78 \mathrm{ppm}(\mathrm{rr})$ & $\sim 0$ \\
\hline $2.57 \mathrm{ppm}(\mathrm{mr})$ & $\sim 0$ \\
\hline $2.45 \mathrm{ppm}(\mathrm{mm})$ & 1.000 \\
\hline $2.27 \mathrm{ppm}(\mathrm{rmr})$ & $\sim 0$ \\
\hline $2.19 / 2.14 \mathrm{ppm}$ & 1.000 \\
\hline $1.55 \mathrm{ppm}(\mathrm{mmm})$ & 1.000 \\
\hline $1.49 \mathrm{ppm}(\mathrm{mmr})$ & $\sim 0$ \\
\hline $1.25 \mathrm{ppm}(\mathrm{rmr})$ & $\sim 0$
\end{tabular}
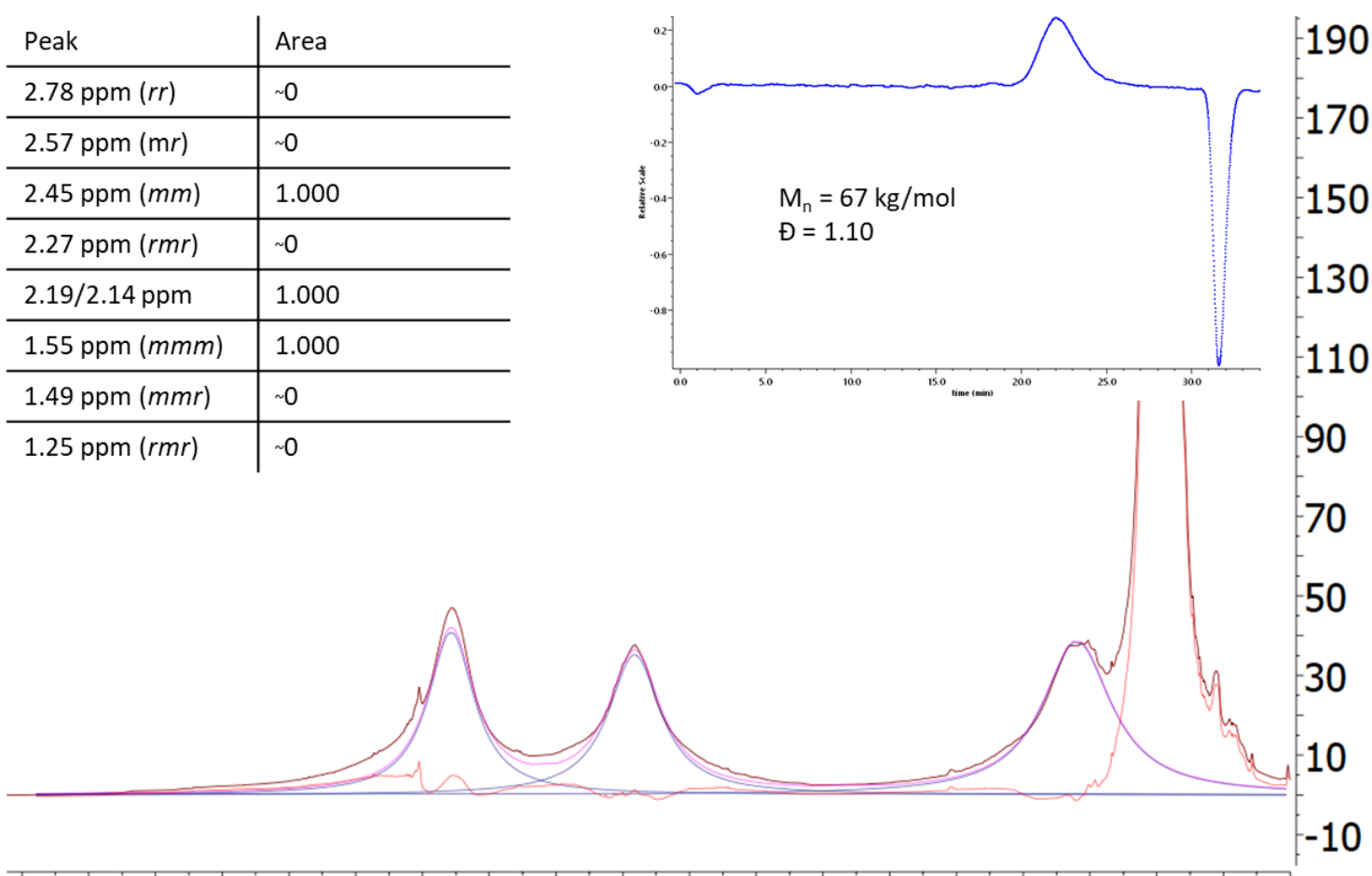

\section{$\begin{array}{llllllllll}3.0 & 2.8 & 2.6 & 2.4 & 2.2 & 2.0 & 1.8 & 1.6 & 1.4 & 1.2\end{array}$ f1 (ppm)}

Figure S17. Quantification of the $\mathrm{mmm}$ tetrads with the line fitting method of MestReNova version 14.0.0-23239 and the GPC trace. Color coding: dark red - original spectrum, blue - peaks, pink - sum, red - residue. 


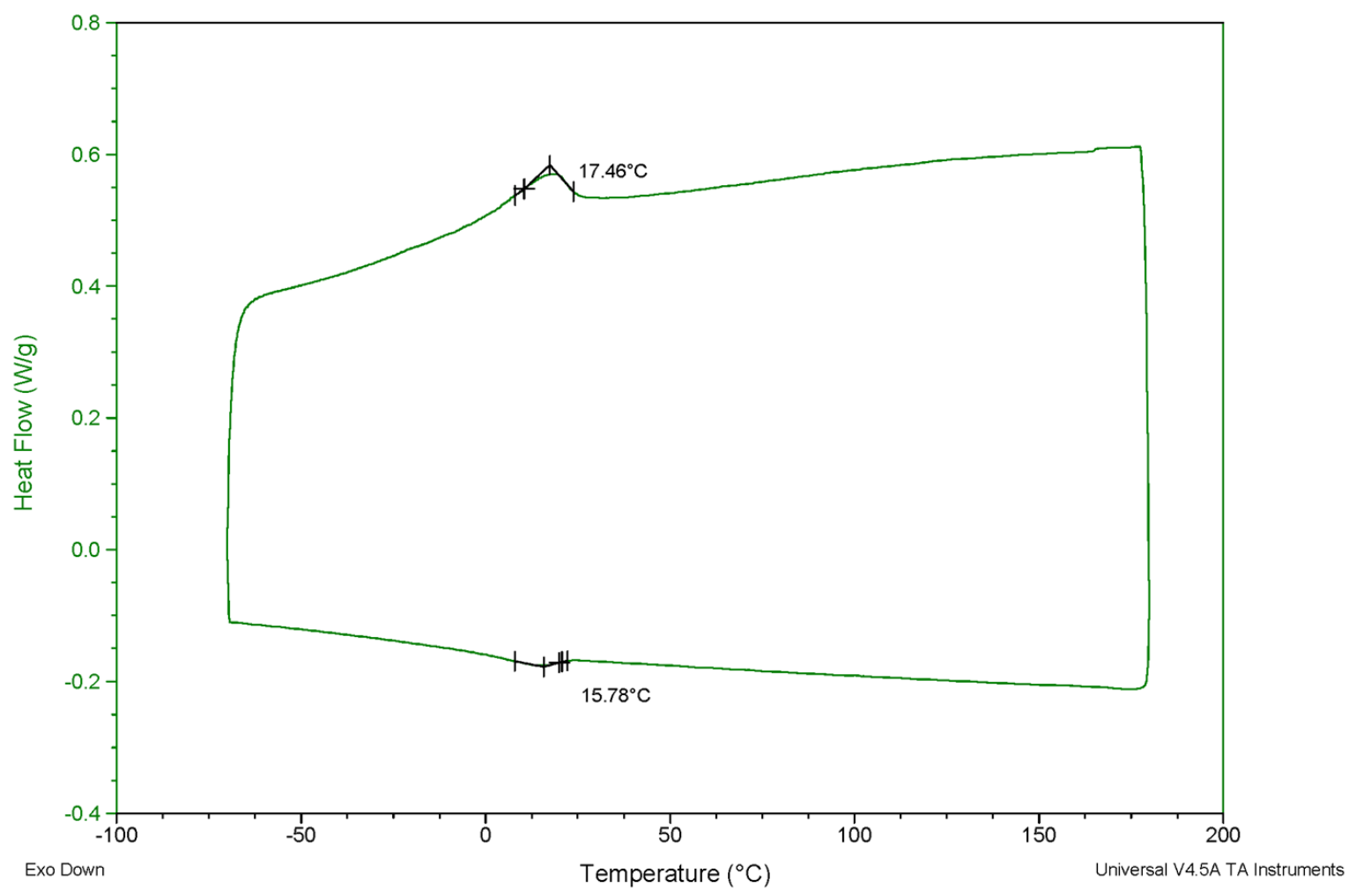

Figure S18. DSC analysis of polymer B showing a melting and crystallization point.

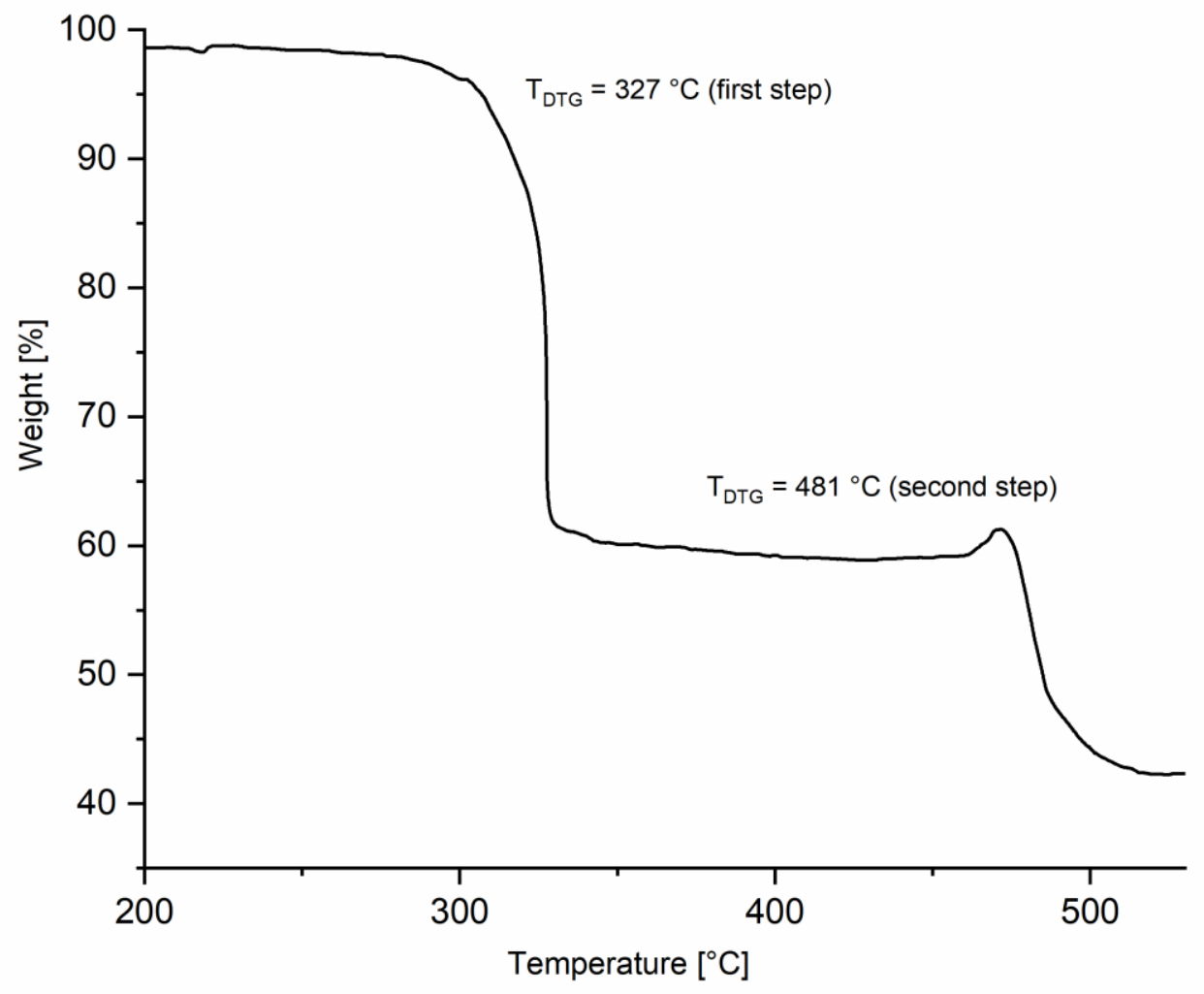

Figure S19. Thermogravimetric analysis of polymer $\mathbf{B}$. 
NMR spectra \& GPC trace of Table 1, run 1:
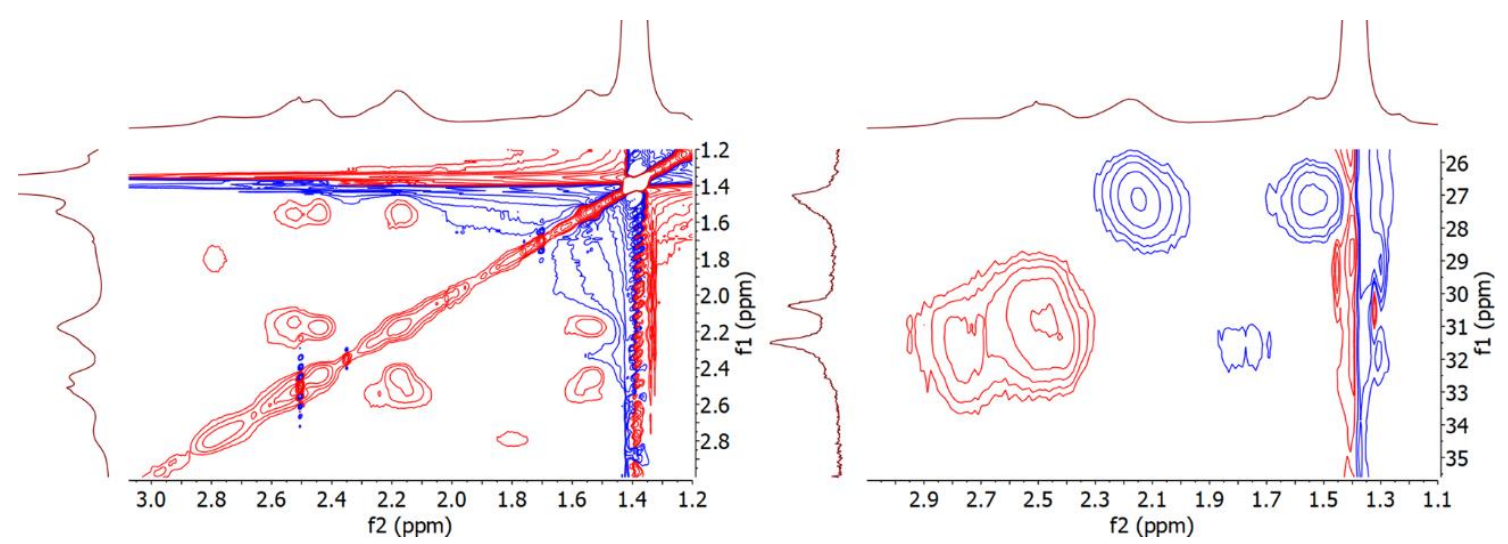

Figure S20. Phase-sensitive ${ }^{1} \mathrm{H}^{-13} \mathrm{C}$ DEPT-edited HSQC (right) and TOCSY $\left\{{ }^{31} \mathrm{P}\right\}$ NMR spectra in $\mathrm{MeOD}_{4}$.

\begin{tabular}{l|l} 
Peak & Area \\
\hline $2.78 \mathrm{ppm}(\mathrm{rr})$ & 0.186 \\
\hline $2.57 \mathrm{ppm}(\mathrm{mr})$ & 0.567 \\
\hline $2.45 \mathrm{ppm}(\mathrm{mm})$ & 0.232 \\
\hline $2.27 \mathrm{ppm}(\mathrm{rmr})$ & 0.260 \\
\hline $2.19 / 2.14 \mathrm{ppm}$ & 1.251 \\
\hline $1.82 \mathrm{ppm}(\mathrm{r})$ & 0.174 \\
\hline $1.55 \mathrm{ppm}(\mathrm{mmm})$ & 1.000 \\
\hline $1.49 \mathrm{ppm}(\mathrm{mmr})$ & 0.120 \\
\hline $1.25 \mathrm{ppm}(\mathrm{rmr})$ & 0.208
\end{tabular}
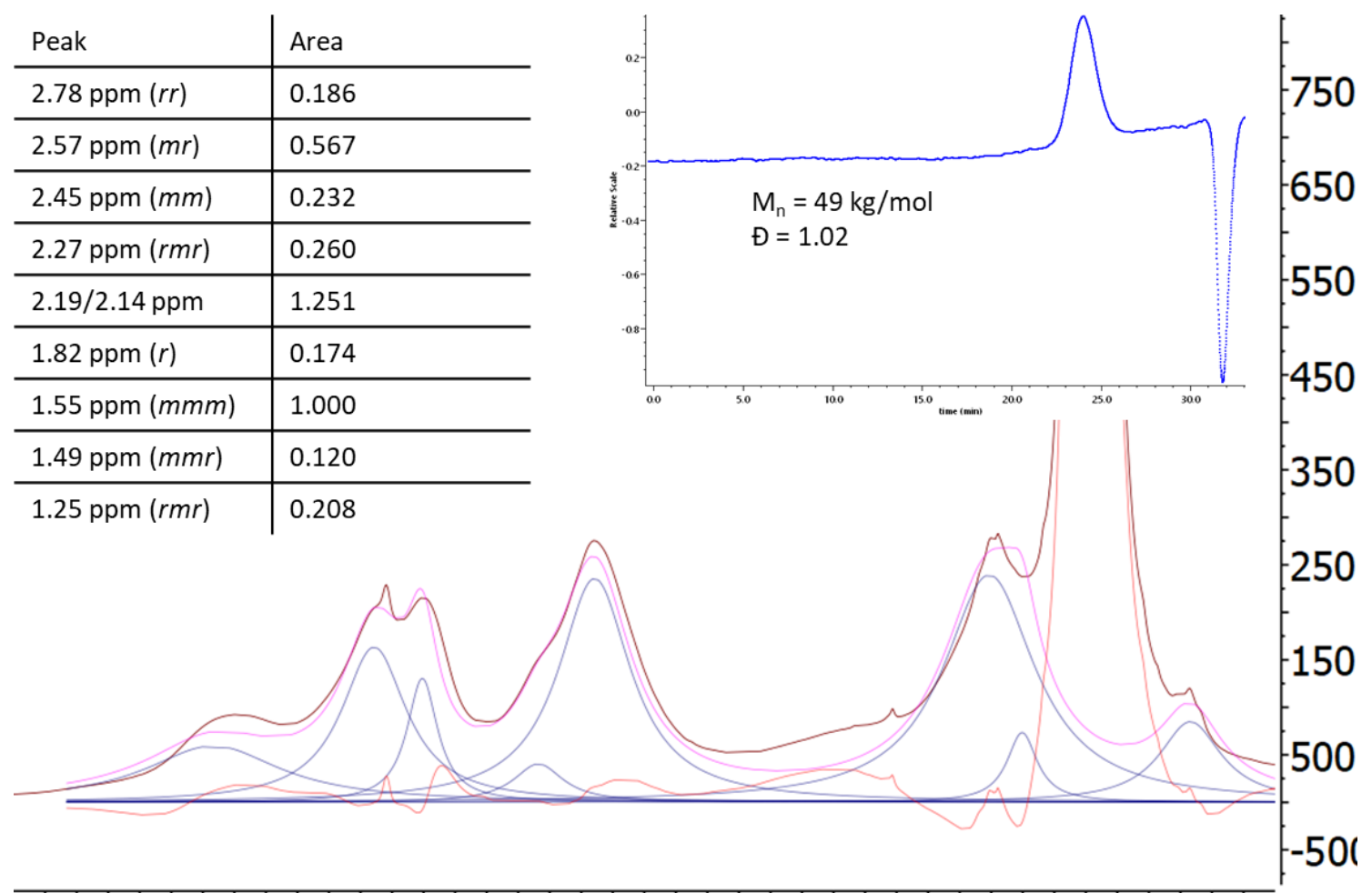

\section{$\begin{array}{llllllllll}3.0 & 2.8 & 2.6 & 2.4 & 2.2 & 2.0 & 1.8 & 1.6 & 1.4 & 1.2\end{array}$ f1 (ppm)}

Figure S21. Quantification of the $\mathrm{mmm}$ tetrads with the line fitting method of MestReNova version 14.0.0-23239 and the GPC trace. Color coding: dark red - original spectrum, blue - peaks, pink - sum, red - residue. 


\section{NMR spectra \& GPC trace of Table 1, run 2:}
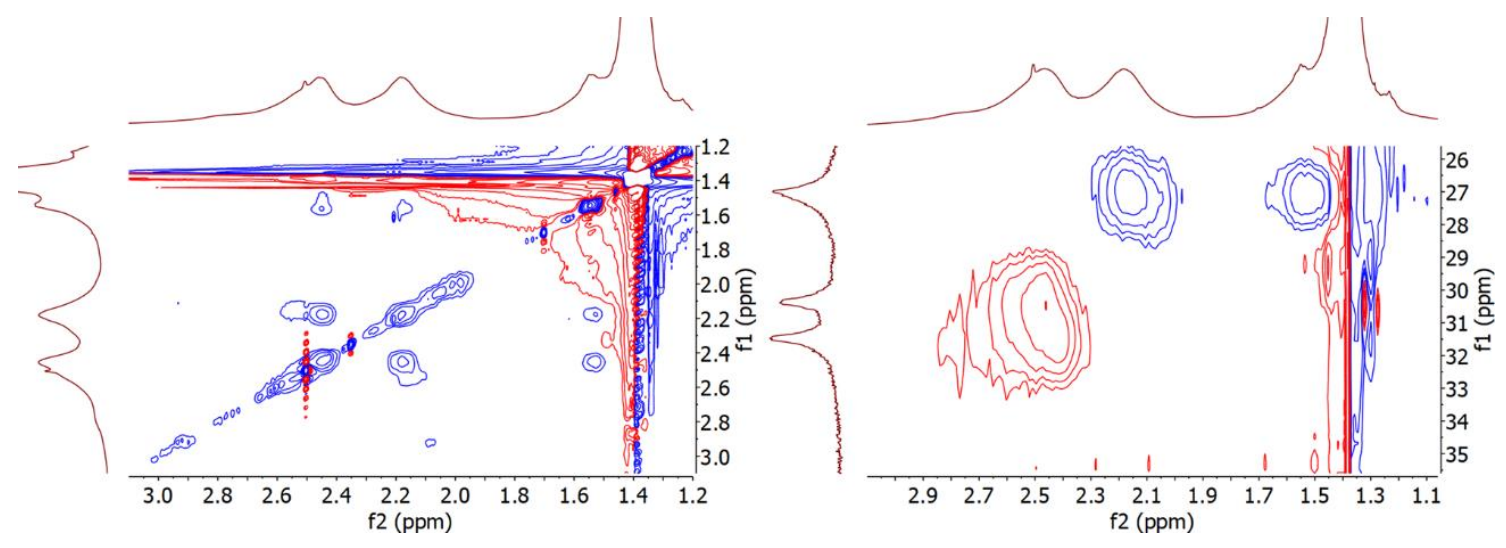

Figure S22. Phase-sensitive ${ }^{1} \mathrm{H}^{13} \mathrm{C}$ DEPT-edited HSQC (right) and TOCSY $\left\{{ }^{31} \mathrm{P}\right\}$ NMR spectra in $\mathrm{MeOD}_{4}$.

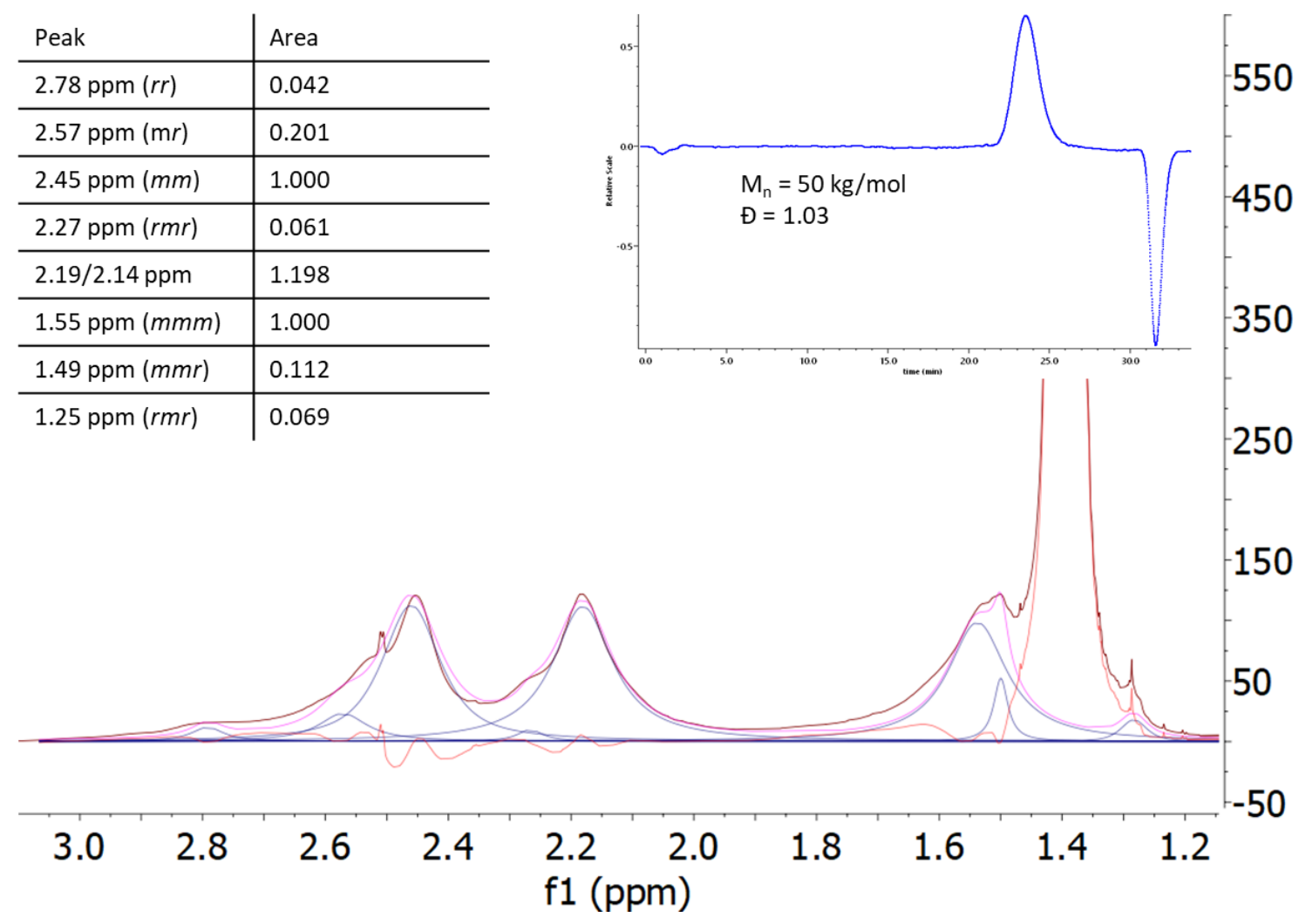

Figure S23. Quantification of the $\mathrm{mmm}$ tetrads with the line fitting method of MestReNova version 14.0.0-23239 and the GPC trace. Color coding: dark red - original spectrum, blue - peaks, pink - sum, red - residue. 


\section{NMR spectra \& GPC trace of Table 1, run 3:}
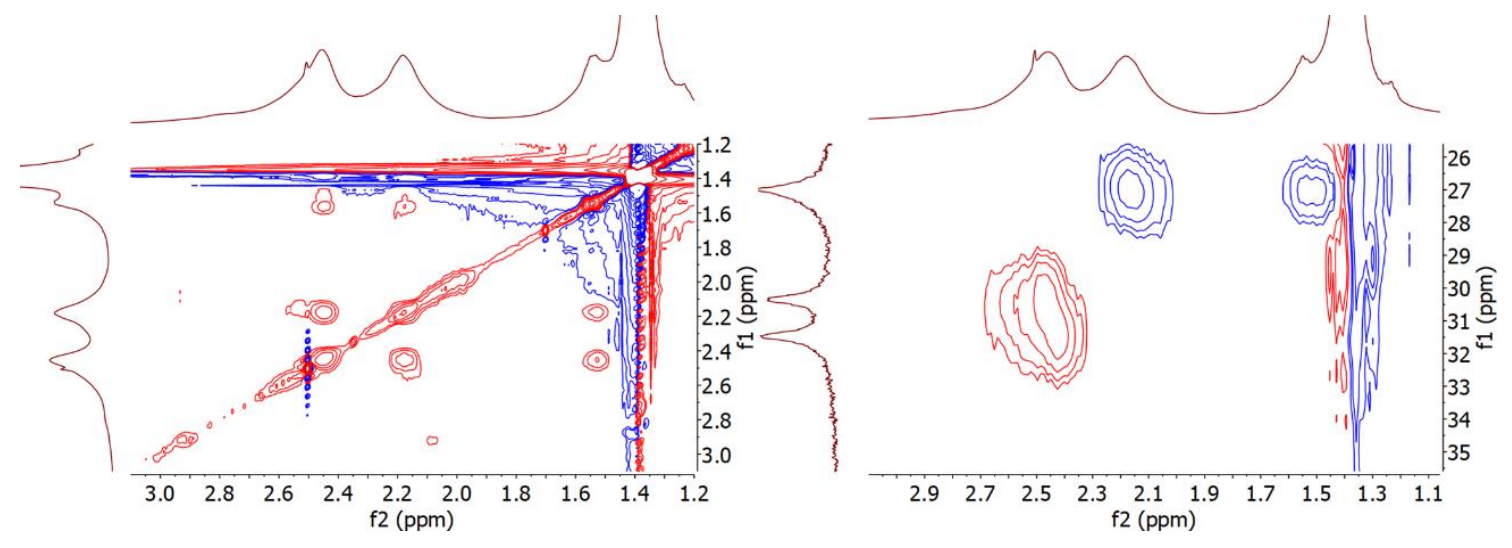

Figure S24. Phase-sensitive ${ }^{1} \mathrm{H}^{13} \mathrm{C}$ DEPT-edited HSQC (right) and TOCSY $\left\{{ }^{31} \mathrm{P}\right\}$ NMR spectra in $\mathrm{MeOD}_{4}$.

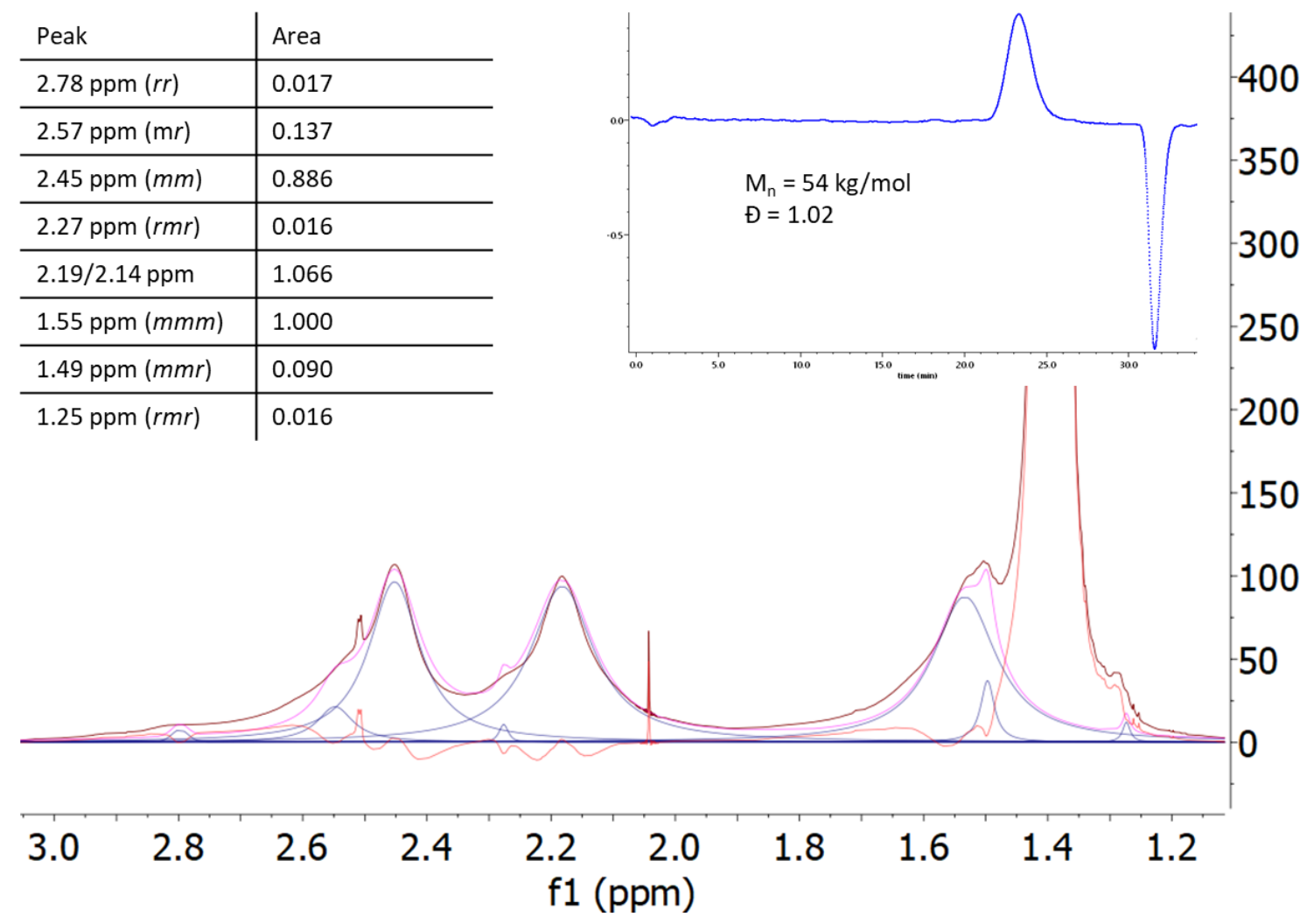

Figure S25. Quantification of the $\mathrm{mmm}$ tetrads with the line fitting method of MestReNova version 14.0.0-23239 and the GPC trace. Color coding: dark red - original spectrum, blue - peaks, pink - sum, red - residue. 


\section{NMR spectra \& GPC trace of Table 1, run 5:}
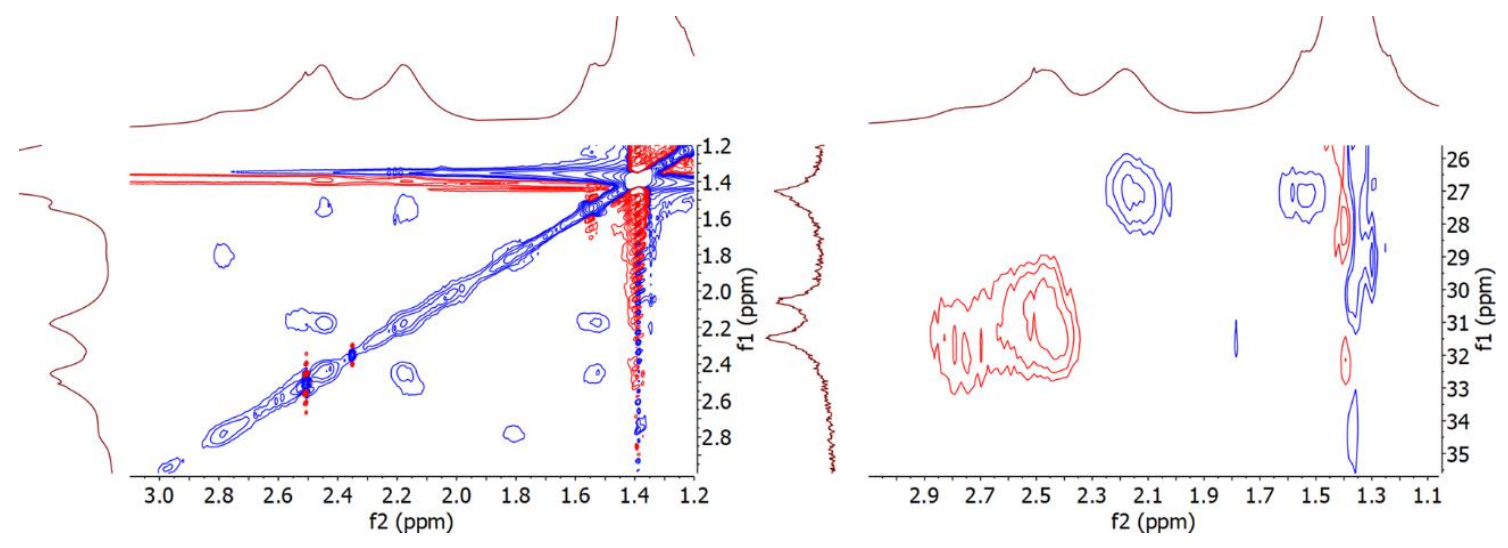

Figure S26. Phase-sensitive ${ }^{1} \mathrm{H}^{13} \mathrm{C}$ DEPT-edited HSQC (right) and TOCSY $\left\{{ }^{31} \mathrm{P}\right\}$ NMR spectra in $\mathrm{MeOD}_{4}$.

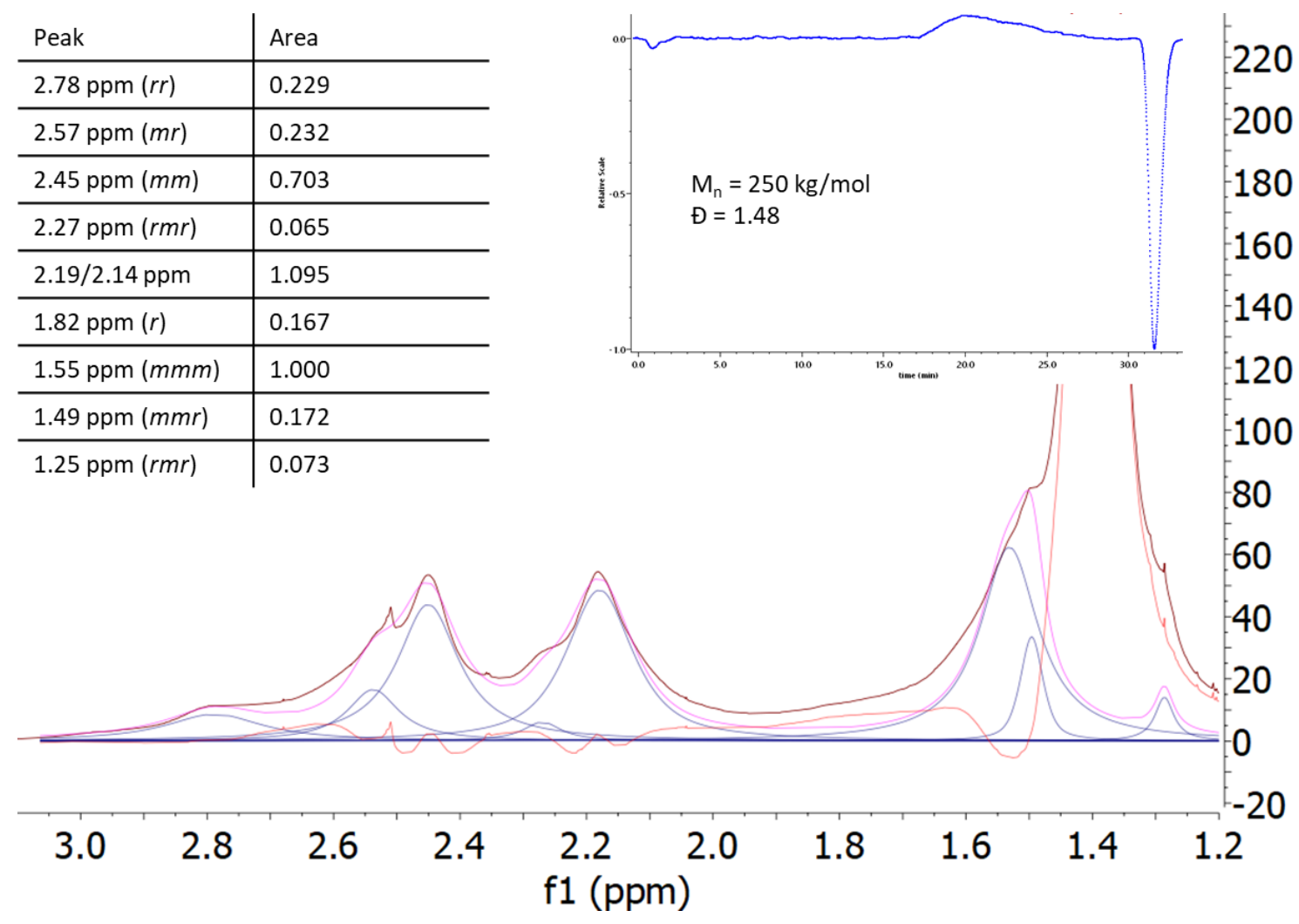

Figure S27. Quantification of the $\mathrm{mmm}$ tetrads with the line fitting method of MestReNova version 14.0.0-23239 and the GPC trace. Color coding: dark red - original spectrum, blue - peaks, pink - sum, red - residue. 
NMR spectra \& GPC trace of Table 1, run 7:
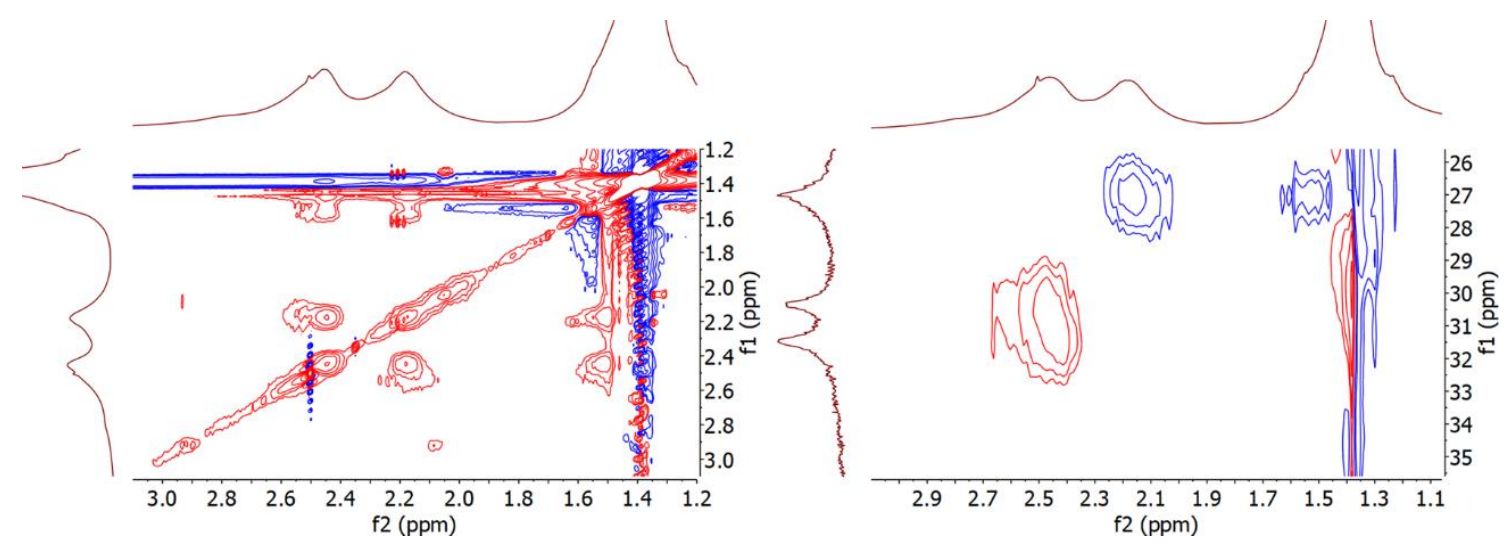

Figure S28. Phase-sensitive ${ }^{1} \mathrm{H}^{13} \mathrm{C}$ DEPT-edited HSQC (right) and TOCSY $\left\{{ }^{31} \mathrm{P}\right\}$ NMR spectra in $\mathrm{MeOD}_{4}$

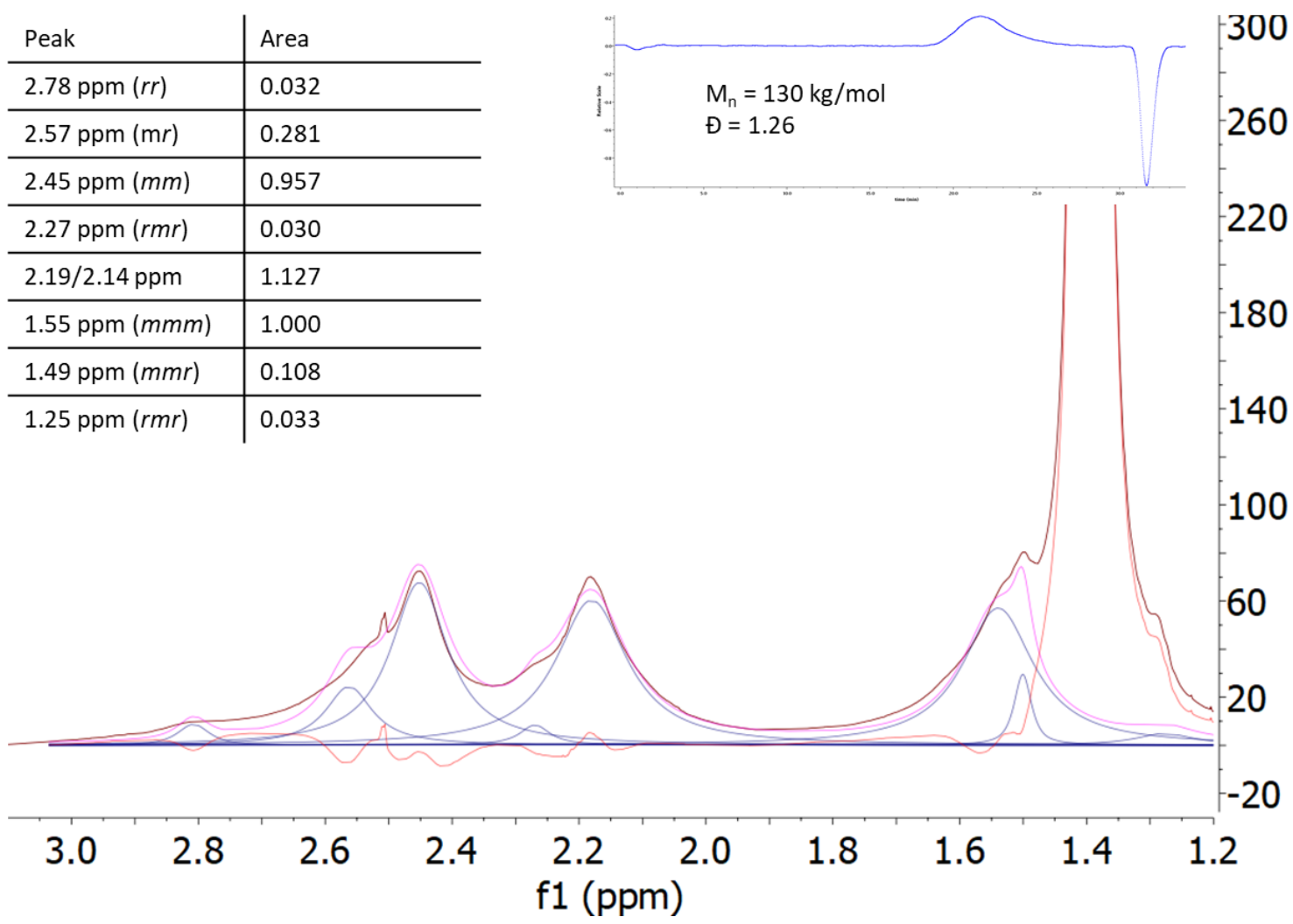

Figure S29. Quantification of the $\mathrm{mmm}$ tetrads with the line fitting method of MestReNova version 14.0.0-23239 and the GPC trace. Color coding: dark red - original spectrum, blue - peaks, pink - sum, red - residue. 
NMR spectra, thermal characteristics \& GPC trace of Table 1, run 8 (polymer B):
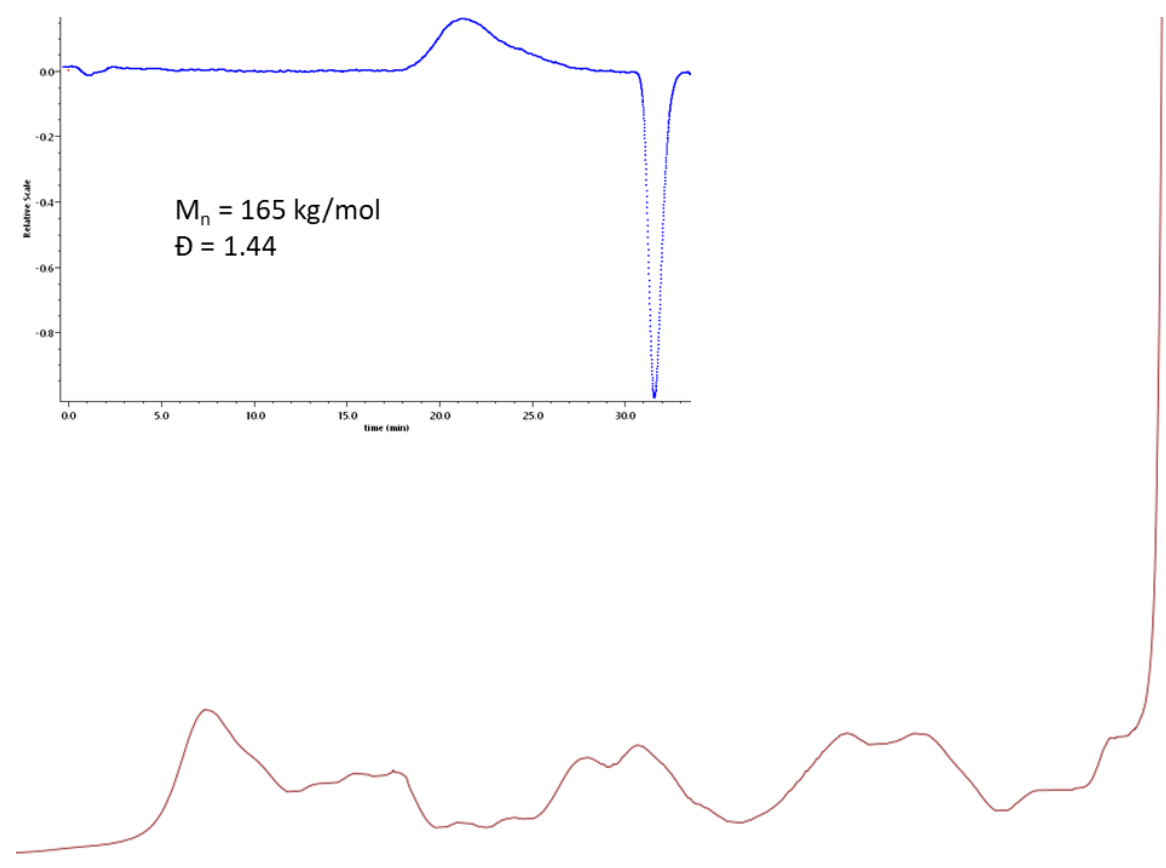

$-50$

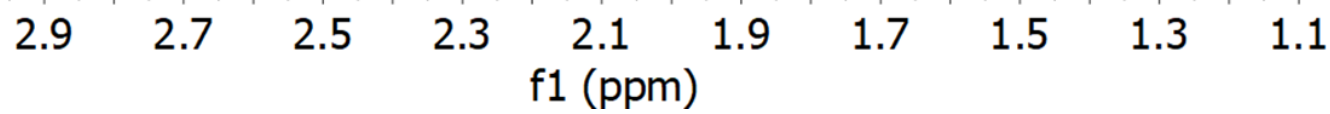

Figure S30. ${ }^{1} \mathrm{H}\left\{{ }^{31} \mathrm{P}\right\}$ NMR spectrum and the GPC trace. 

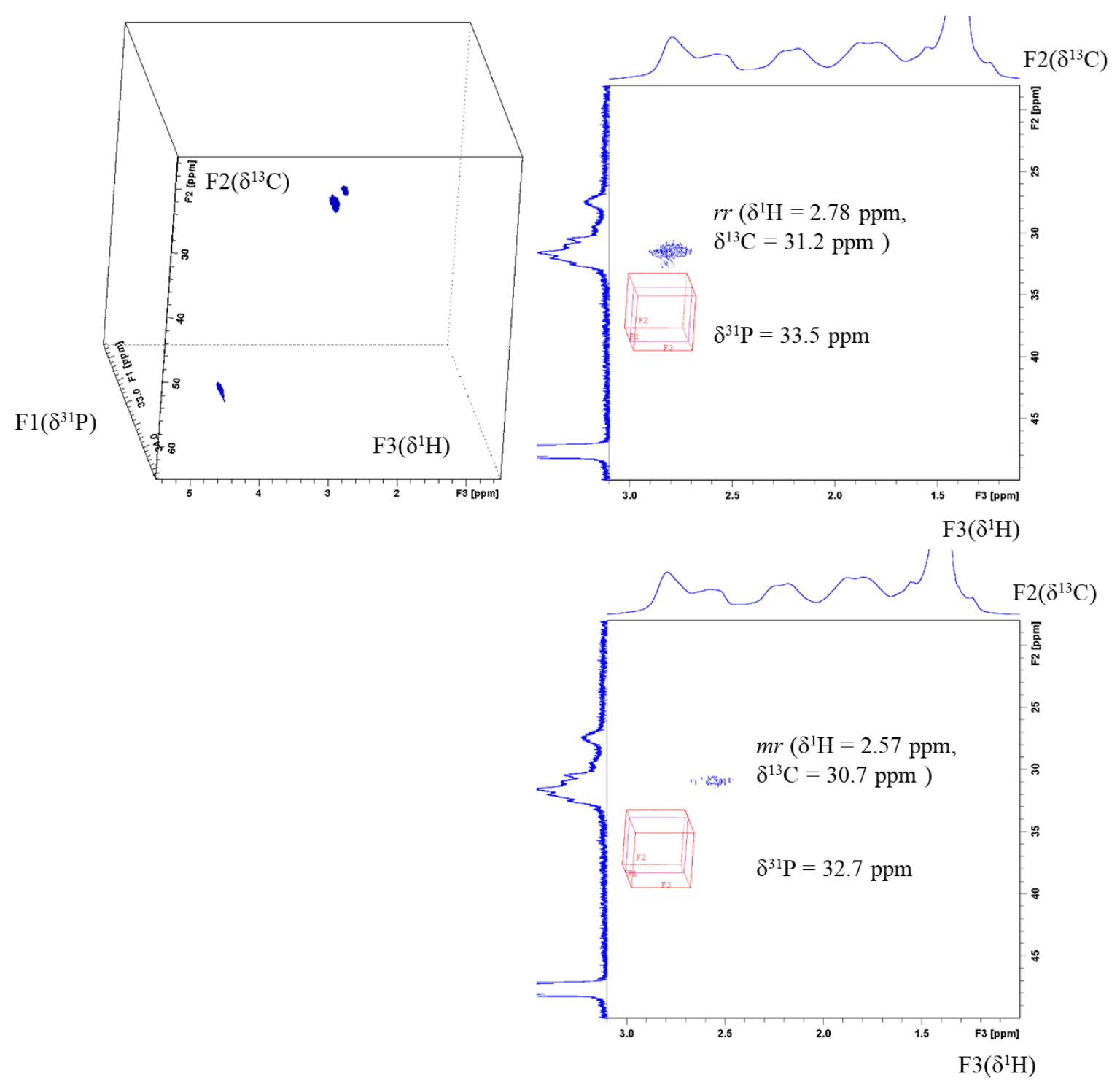

Figure S31. ${ }^{1} \mathrm{H}^{-13} \mathrm{C}-{ }^{31} \mathrm{P}$ triple resonance HCP 3D experiment edited by $J_{\mathrm{PC}}=110 \mathrm{~Hz}$ (for details see Table S11, S12). 


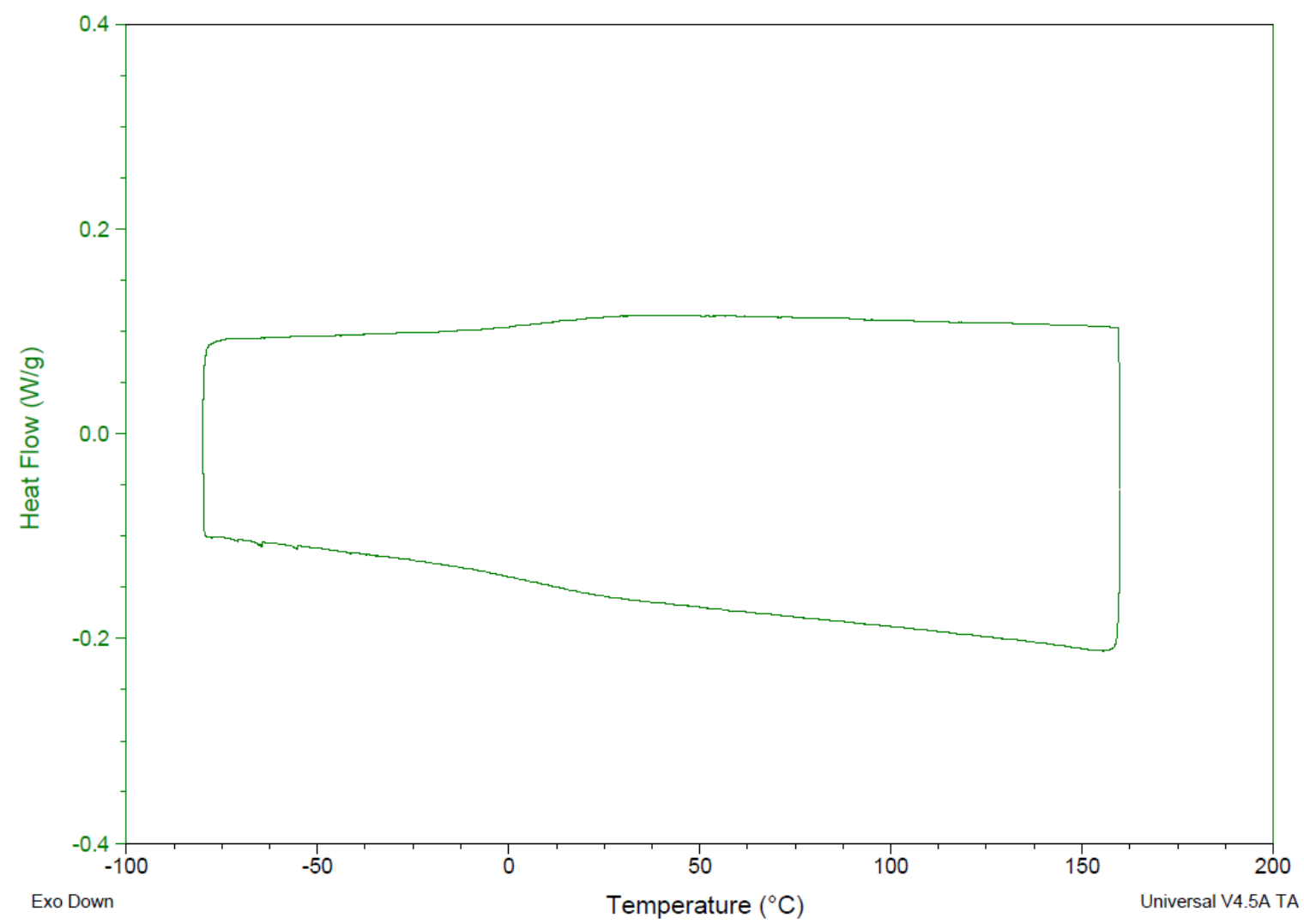

Figure S32. DSC analysis of polymer A showing no melting point.

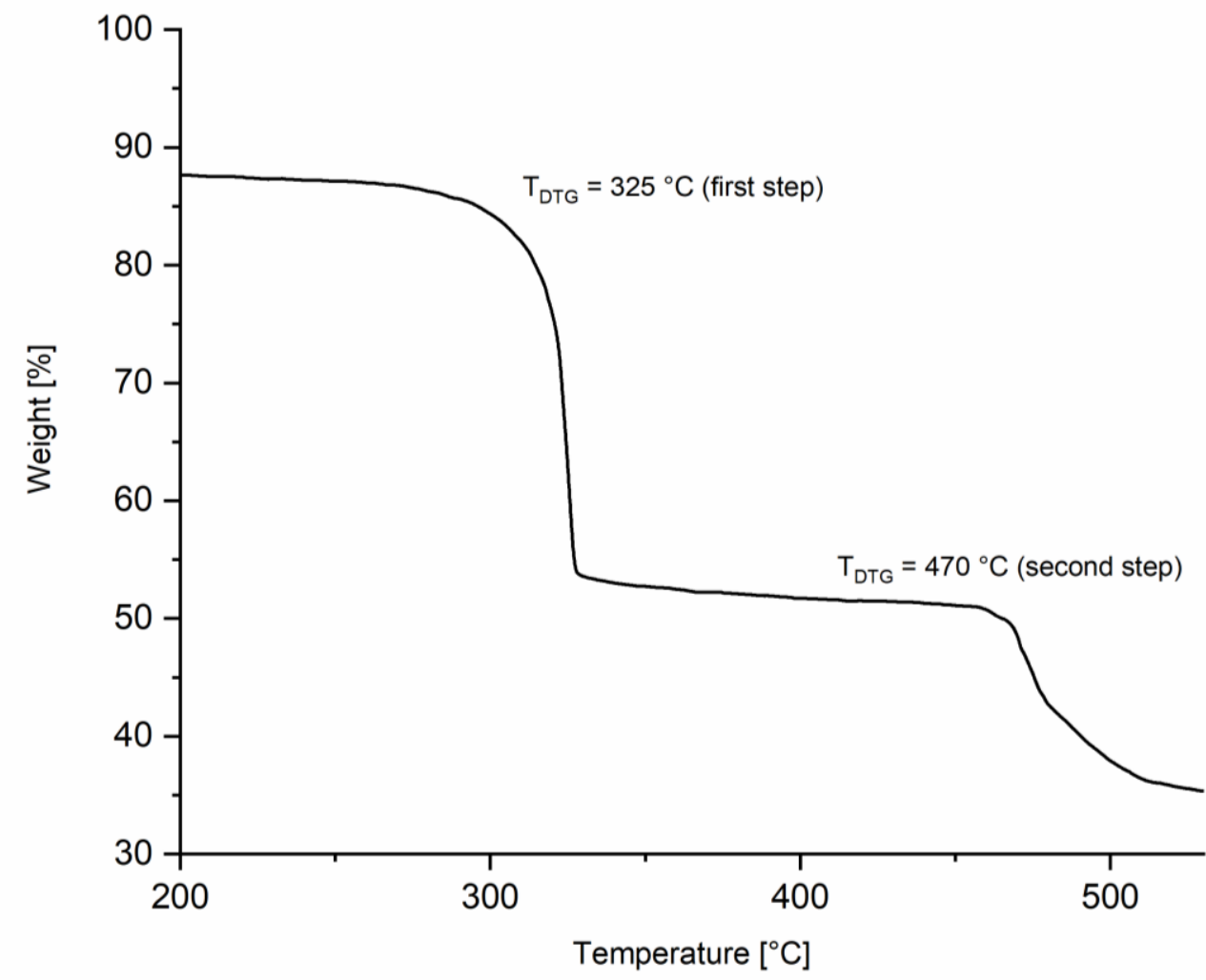

Figure S33. Thermogravimetric analysis of polymer A. 
NMR spectra \& GPC trace of Table 1, run 10:

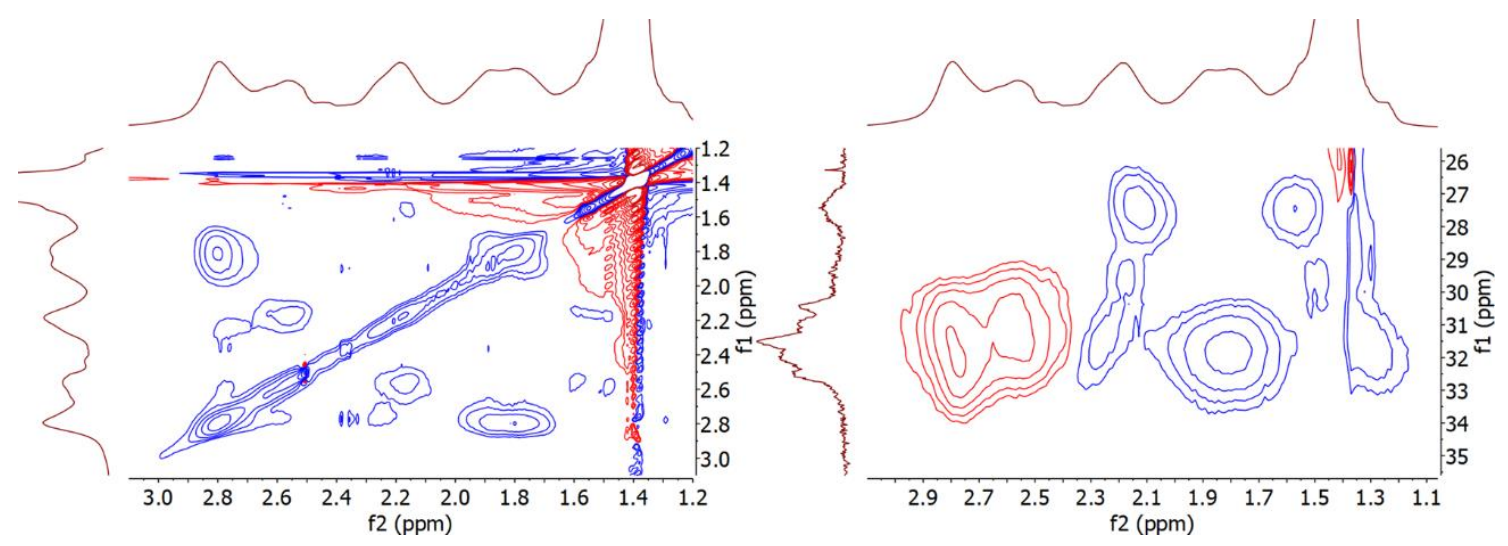

Figure S34. Phase-sensitive ${ }^{1} \mathrm{H}^{-13} \mathrm{C}$ DEPT-edited HSQC (right) and TOCSY $\left\{{ }^{31} \mathrm{P}\right\}$ NMR spectra in $\mathrm{MeOD}_{4}$.

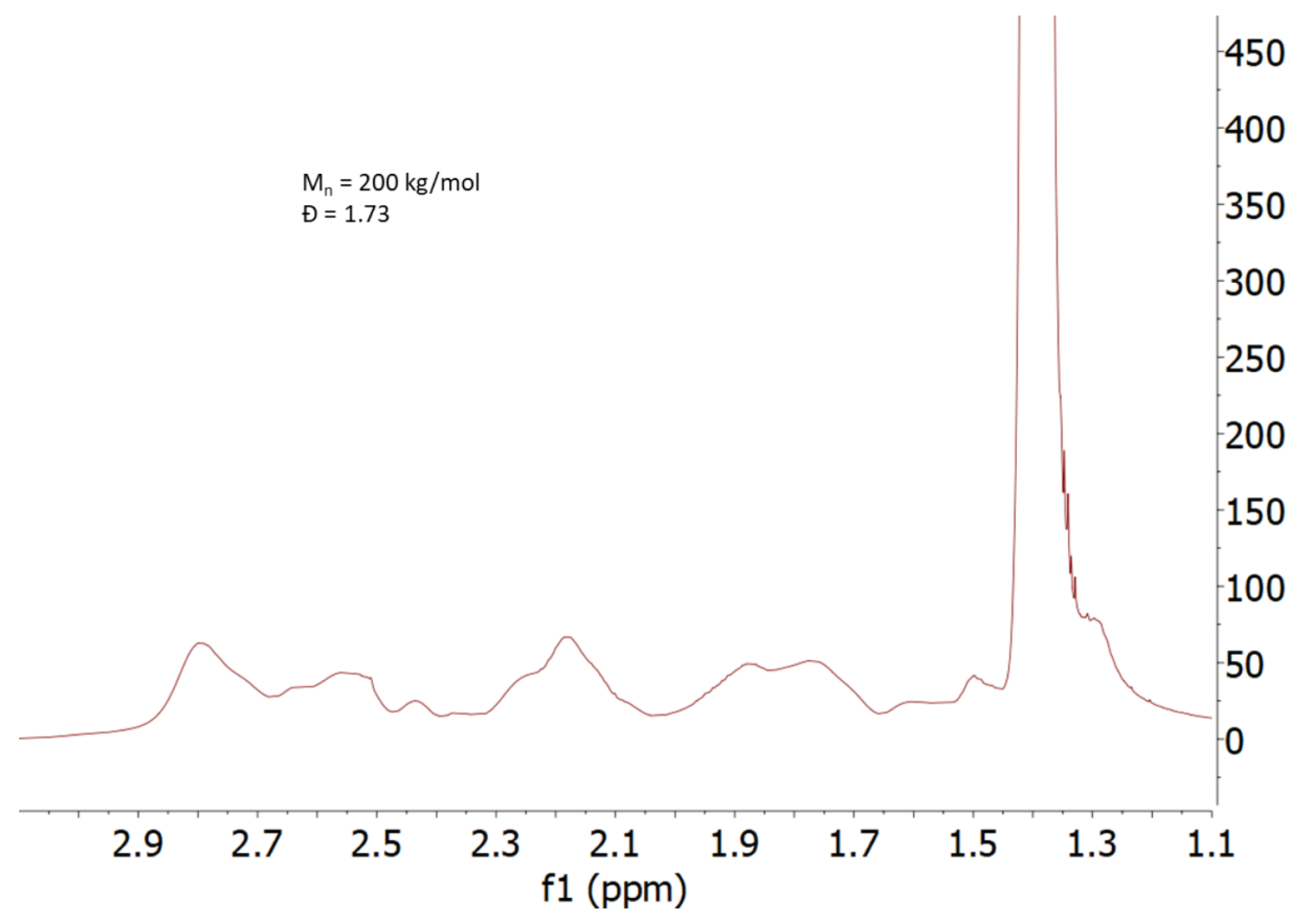

Figure S35. ${ }^{1} \mathrm{H}\left\{{ }^{31} \mathrm{P}\right\}$ NMR spectrum and the GPC trace. 
Saponification of Table 1, run 4 (polymer B):

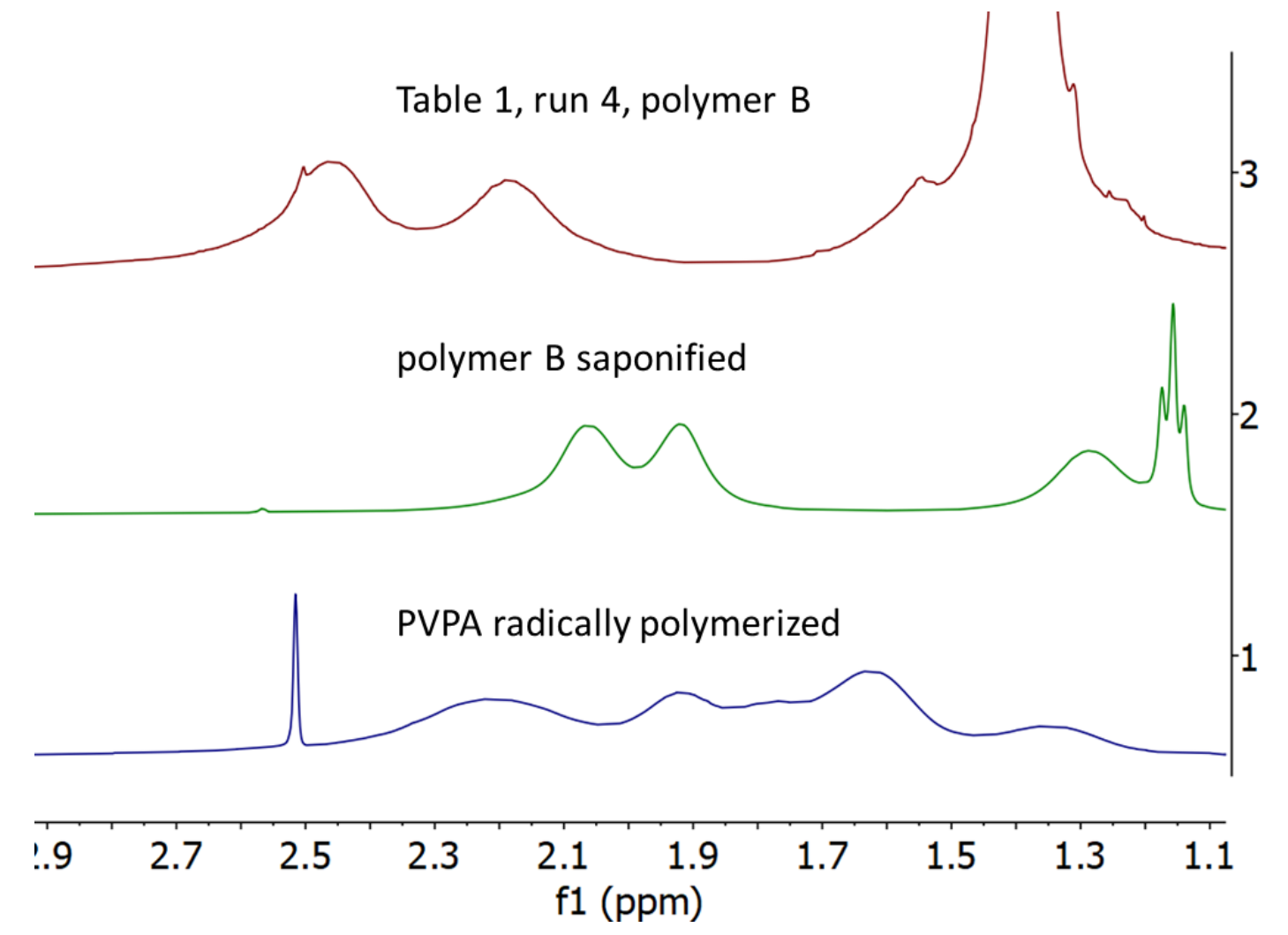

Figure S36. ${ }^{1} \mathrm{H}$ NMR spectra of polymer $\mathrm{B}$ in $\mathrm{MeOD}_{4}$ and its saponification product in $\mathrm{D}_{2} \mathrm{O}(1.10 \mathrm{~g} / \mathrm{L}$ DEVP in DCM + excess TMSBr 24 h reflux; $2.1 \mathrm{~m} \mathrm{HCl} 2 \mathrm{~h}$ r.t. and aqueous dialysis) compared to a radically produced PVPA in $\mathrm{D}_{2} \mathrm{O}$. 


\section{References}

(1) Behr, A. Angewandte homogene Katalyse; Wiley-VCH Verlag GmbH \& Co. KGaA: Weinheim, 2008.

(2) van Leusen, D.; Beetstra, D. J.; Hessen, B.; Teuben, J. H. Ethylene-Bridged

Tetramethylcyclopentadienylamide Titanium Complexes: Ligand Synthesis and Olefin Polymerization Properties $\uparrow$. Organometallics 2000, 19, 4084-4089, DOI: 10.1021/om000402f.

(3) Resconi, L.; Camurati, I.; Grandini, C.; Rinaldi, M.; Mascellani, N.; Traverso, O. Indenyl-amido titanium and zirconium dimethyl complexes: improved synthesis and use in propylene polymerization. J. Organomet. Chem. 2002, 664, 5-26, DOI: 10.1016/S0022-328X(02)01853-3.

(4) Hou, Z.; Koizumi, T.-a.; Nishiura, M.; Wakatsuki, Y. Lanthanide(II) Complexes Bearing Linked Cyclopentadienyl-Anilido Ligands: Synthesis, Structures, and One-Electron-Transfer and Ethylene Polymerization Reactions. Organometallics 2001, 20, 3323-3328, DOI: 10.1021/om010261x.

(5) Carpenetti, D. W.; Kloppenburg, L.; Kupec, J. T.; Petersen, J. L. Application of Amine Elimination for the Efficient Preparation of Electrophilic ansa-Monocyclopentadienyl Group 4 Complexes Containing an Appended Amido Functionality. Structural Characterization of $\left[\left(\mathrm{C}_{5} \mathrm{H}_{4}\right) \mathrm{SiMe}_{2}(\mathrm{~N}-t-\mathrm{Bu})\right] \mathrm{ZrCl}_{2}\left(\mathrm{NMe}_{2} \mathrm{H}\right)$. Organometallics 1996, 15, 1572-1581, DOI: 10.1021/om950858a.

(6) Hultzsch, K. C.; Spaniol, T. P.; Okuda, J. Half-Sandwich Alkyl and Hydrido Complexes of Yttrium: Convenient Synthesis and Polymerization Catalysis of Polar Monomers. Angew. Chem. Int. Ed. 1999, 38, 227-230, DOI: 10.1002/(SICI)1521-3773(19990115)38:1/2<227:AIDANIE227>3.0.CO;2-M.

(7) Hultzsch, K. C.; Voth, P.; Beckerle, K.; Spaniol, T. P.; Okuda, J. Single-Component Polymerization Catalysts for Ethylene and Styrene: Synthesis, Characterization, and Reactivity of Alkyl and Hydrido Yttrium Complexes Containing a Linked Amido-Cyclopentadienyl Ligand. Organometallics 2000, 19, 228-243, DOI: 10.1021/om990583p. 